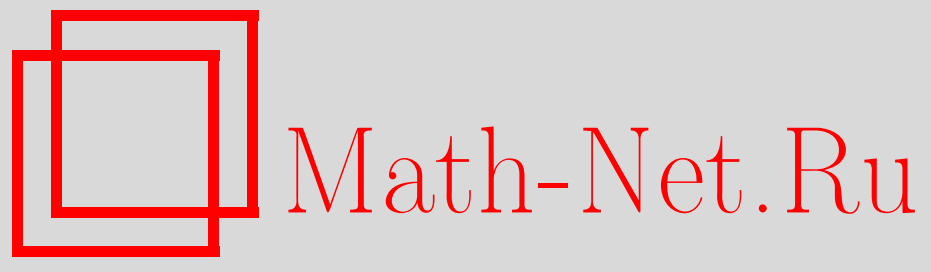

А. М. Вершик, М. И. Граев, Интегральные модели представлений групп токов простых групп Ли, УМН, 2009, том 64, выпуск 2, 5-72

DOI: https://doi.org/10.4213/rm9260

Использование Общероссийского математического портала Math-Net.Ru подразумевает, что вы прочитали и согласны с пользовательским соглашением http://www . mathnet.ru/rus/agreement

Параметры загрузки:

IP : 54.80 .97 .219

26 апреля 2023 г., 04:47:59

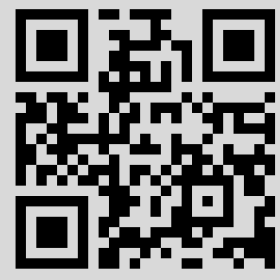




\title{
Интегральные модели представлений групп токов простых групп Ли
}

\author{
А. М. Вершик, М. И. Граев
}

Для класса локально компактных групп, представимых в виде полупрямого произведения локально компактной группы и однопараметрической группы ее автоморфизмов $\mathbb{R}_{+}^{*}$, строится новая модель представлений их групп токов. Конструкция применяется к максимальным параболическим подгруппам всех простых групп ранга 1. Для случая групп $\mathrm{SO}(n, 1)$ и $\mathrm{SU}(n, 1)$ построено продолжение представлений групп токов их максимальных параболических подгрупп до представлений групп токов самих групп. Главную роль в конструкции играет $\sigma$-конечная мера (бесконечномерная мера Лебега в пространстве распределений).

Библиография: 32 названия.

Ключевые слова: группа токов, интегральная модель, фоковское представление, каноническое представление, особое представление, бесконечномерная лебегова мера.

\section{СОДЕРЖАНИЕ}

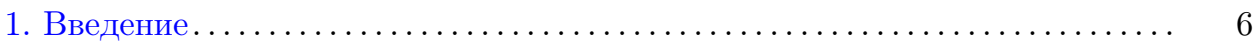

2. Мера $\mathscr{L}$ в пространстве распределений .................... 11

3. Канонические представления группы $P_{0}$ и ассоциированные с ними

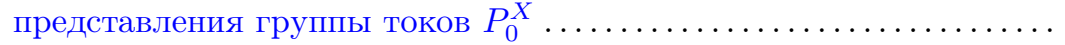

4. Представления группы $P=\mathbb{R}_{+}^{*} \curlywedge P_{0}$ и ее группы токов $P^{X}$, ассоциированные с каноническими представлениями подгруппы $P_{0} \ldots \ldots \ldots$

5. Интегральная модель представления группы токов $\mathrm{O}(n, 1)^{X}, n>2 \ldots 31$

6. Интегральные модели представлений группы токов $\operatorname{SL}(2, \mathbb{R})^{X} \ldots \ldots \ldots$

7. Интегральные модели представлений группы токов $\mathrm{U}(n, 1)^{X} \ldots \ldots \ldots \quad 51$

8. Интегральные модели представлений группы $P^{X}$, где $P$ - максимальная параболическая подгруппа группы $\operatorname{Sp}(n, 1) \ldots \ldots \ldots \ldots \ldots \ldots \ldots \ldots . \ldots \ldots$

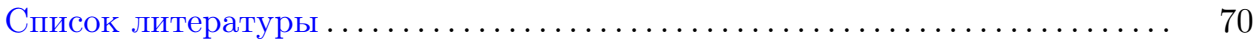

Первый автор поддержан РФФИ (грант № 08-01-00379) и программой "Ведущие научные школы” (грант НШ 2460.2008.1112); второй автор поддержан РФФИ (грант № 07-01-00101a).

(C) А. М. ВЕРшик, М. И. Граев, 2009 


\section{1. Введение}

1.1. Построение инвариантного мультипликативного интеграла представлений, т. е. неприводимого представления группы токов $L^{\infty}(X ; G) \equiv G^{X}$ - ограниченных измеримых функций на пространстве с мерой $(X, m)$ - со значениями в полупростой группе Ли $G$ было предпринято в начале 1970 -х годов в работах [1], [2]. Позднее выяснилось, что это построение вкладывается в общую схему, описанную несколькими годами ранее Х. Араки в терминах пространства Фока (см. [3]). Однако, как отмечает сам автор [3], схема была применена только к разрешимым и нильпотентным группам Ли, а полупростые группы не рассматривались. Формально речь идет о некоммутативном аналоге безгранично делимых мер, т. е. о полугруппах состояний на группах, и об аналоге формул Леви-Хинчина, но весьма специального вида. Ключевой момент состоит в нахождении нетривиальных когомологий группы со значениями в подходящем неприводимом унитарном представлении. Построение интеграла представлений для группы $\mathrm{SL}(2, \mathbb{R})$, данное в работе [1], неявно содержало необходимый коцикл. Явное описание когомологий для $\mathrm{SL}(2, \mathbb{R})$ и других простых групп Ли приведено в книге [4].

Наличие неприводимого унитарного представления $\pi$ группы $G$, для которого $H^{1}(G ; \pi) \neq 0$, есть достаточное, а в схеме Араки (т. е. для фоковской факторизации) и необходимое условие существования мультипликативного интеграла представлений. В свою очередь, это условие означает, что группа $G$ не должна обладать свойством (Т) Каждана [5] (см. книгу [6] и библиографию в ней), т. е. тривиальное представление не должно быть изолированным в пространстве неприводимых унитарных представлений в топологии Фелла. Действительно, как доказано в [7], представление $\pi$ с ненулевой группой $H^{1}(G ; \pi)$ должно быть "приклеено" к тривиальному (по терминологии [7] - быть бесконечно малым). Среди классических простых групп Ли $G$ лишь у групп $\mathrm{SO}(n, 1), n>1$, и $\mathrm{SU}(n, 1), n \geqslant 1$, есть такие унитарные представления, и только они допускают инвариантный мультипликативный интеграл представлений в фоковской модели.

Анализ первоначальных работ [1], [2], [8]-[10] показал, что имеется альтернативный подход к описанию фоковских представлений. Сначала он появился всего лишь как результат диагонализации обсуждаемых в [1] представлений для группы $\mathrm{SL}(2, \mathbb{R})$ относительно унипотентной подгруппы [11], [12]. В качестве необходимого следствия этой диагонализации возникло определение замечательной сигма-конечной меры в пространстве дискретных мер на $X$. Однако истинная суть и глубина альтернативного описания мультипликативного интеграла представлений выявилась лишь после того, как был разобран общий случай групп $\mathrm{SO}(n, 1), n>1$, и $\mathrm{SU}(n, 1), n \geqslant 1$. Этому посвящены работы [13]-[16] и об этом пойдет речь в настоящей статье. В ней мы подводим итог этому циклу работ и рассматриваем ее как подготовительную к монографии на тему о представлениях групп токов. В отличие от фоковской модели, альтернативная модель, которая названа по некоторым причинам интегральной, существенно использует специфические свойства простых групп ранга 1, точнее их максимальных параболических подгрупп, и инвариантность некоторой сигма-конечной меры относительно континуальной картановской группы. 
Поэтому она не обладает общностью фоковской модели, а ее изоморфизм с фоковской моделью очень сложен. Но, во-первых, она позволяет дать значительно более простое доказательство неприводимости и других свойств представления, а во-вторых, она приводит к новой наглядной трактовке понятия непрерывного тензорного произведения, что несомненно будет полезно в дальнейшем.

\section{2. Краткое введение в фоковскую и интегральную модели.}

1.2.1. Опишем сначала исходную - фоковскую - модель во всей общности. Она основана на конструкции экспоненты, являющейся формализацией, с одной стороны, фоковского пространства, а с другой - пространства $L^{2}$ по гауссовой мере (пространства Винера-Ито).

Экспонента гильбертова пространства $H$ определяется с помощью разложения:

$$
\mathscr{H} \equiv \operatorname{EXP} H=\mathbb{C} \oplus H \oplus \frac{1}{\sqrt{2 !}} S^{2} H \oplus \frac{1}{\sqrt{3 !}} S^{2} H \oplus \cdots,
$$

где $S^{n} H-n$-я симметрическая тензорная степень пространства $H$, и вводится отображение $\exp : H \rightarrow \operatorname{EXP} H$ :

$$
\exp h=1 \oplus h \oplus \frac{1}{\sqrt{2 !}} h \otimes h \oplus \frac{1}{\sqrt{3 !}} h \otimes h \otimes h \oplus \cdots \in \mathscr{H} \quad \text { при } h \in H .
$$

При этом имеют место соотношения:

$$
\begin{gathered}
\operatorname{EXP}\left(H_{1} \oplus H_{2}\right)=\operatorname{EXP} H_{1} \otimes \operatorname{EXP} H_{2}, \\
\left\langle\exp h_{1}, \exp h_{2}\right\rangle=e^{\left(h_{1}, h_{2}\right)}, \quad \exp \left(h_{1}+h_{2}\right)=\exp h_{1} \otimes \exp h_{2} .
\end{gathered}
$$

Экспоненты $\exp h$ образуют тотальное множество в $\mathscr{H}$, т. е. их линейная оболочка всюду плотна в $\mathscr{H}$. С помощью экспонент задается вся структура фоковского пространства: разложение на многочастичные подпространства, операторы рождения и уничтожения и т. п. Унитарные операторы, действующие в этом пространстве и сохраняющие его структуру, называются факторизованными. Они параметризуются тройками $(A, b, c)$, где $A$ - унитарный оператор в $H$, $b \in H, c \in \mathbb{C},|c|=1$, и образуют группу: $\mathscr{A}=\{(A, b, c): A \in \operatorname{Unit}(H), b \in H$, $c \in \mathbb{C},|c|=1\}$ с законом умножения

$$
\left(A_{1}, b_{1}, c_{1}\right)\left(A_{2}, b_{2}, c_{2}\right)=\left(A_{1} A_{2}, b_{1}+A_{1} b_{2}, c_{1} c_{2} \exp \left(i \operatorname{Im}\left\langle b_{1}, A_{1} b_{2}\right\rangle\right)\right) .
$$

Действие ее на экспонентах задается следующим образом:

$$
(A, b, c)(\exp h)=c \exp (i \operatorname{Im}\langle b, h\rangle) \cdot \exp (A h+b) .
$$

Таким образом, группа $\mathscr{A}$, иногда называемая группой Боголюбова, есть центральное расширение группы изометричных движений пространства $\mathscr{H}$ (т. е. полупрямого произведения группы $\operatorname{Unit}(H)$ и группы трансляций $P$ ).

Нетрудно видеть, что представление группы токов $G^{X}=L^{\infty}(X, G)$ задает факторизацию в пространстве представления. Если предположить, что эта факторизация фоковская, то представление пропускается через группу факторизованных операторов - группу Боголюбова. В предположении инвариантности представления относительно группы всех преобразований пространства $X$, 
сохраняющих меру, мы немедленно получаем, что это представление параметризуется унитарным представлением $\pi$ самой группы $G$ в пространстве $H$ и 1 -коциклом $\beta: G \rightarrow H$ группы $G$ со значениями в $H$. Для неприводимости представления группы токов $G^{X}$ достаточно, чтобы представление $\pi$ было неприводимым, а коцикл $\beta$ был некогомологичным нулю (см. [2], [12], [17], [18]). Эта общая схема никак не использует специфику групп. Задача сводится к поискам пар $\pi, \beta$ для данной группы или к доказательству, что таких пар нет. Это и приводит к упомянутому ответу для простых групп Ли, поскольку среди простых групп Ли особые представления с нетривиальной группой $H^{1}(G ; \pi)$ существуют только у групп $\mathrm{SO}(n, 1), n>1$, и $\mathrm{SU}(n, 1), n \geqslant 1$.

Для удобства во всех построениях группь $\mathrm{SO}(n, 1)$ и $\mathrm{SU}(n, 1)$ заменяются их расширениями $\mathrm{O}(n, 1)$ u $\mathrm{U}(n, 1)$.

Отметим, что особые представления как самих групп $\mathrm{SO}(n, 1)$ и $\mathrm{SU}(n, 1)$, так и их расширений $\mathrm{O}(n, 1)$ и $\mathrm{U}(n, 1)$ тривиальны на их иентре, а потому сводятся к представлениям проективизачий этих групп. Соответственно фоковские представления их групп токов $G^{X}$ сводятся к представлениям проективизаций $G^{X}$.

1.2.2. Изучаемая далее для групп $G=\mathrm{O}(n, 1), n>1$, и $G=\mathrm{U}(n, 1), n \geqslant 1$, интегральная модель представлений их групп токов существенно опирается на структуру самих групп $G$. При этом, не используя само существование представлений группы токов и не рассматривая их группы когомологий, мы, наоборот, получаем попутно всю эту информацию. Для нас важны два фундаментальных факта, относящихся к группам $G=\mathrm{O}(n, 1), n>1$, и $\mathrm{U}(n, 1)$, $n \geqslant 1 .^{1}$

(А) Неприводимые особые унитарные представления этих групп остаются неприводимыми при ограничении на максимальную параболическую подгруппу.

(B) Максимальная параболическая подгруппа этих групп $P$ есть полупрямое произведение мультипликативной группы $\mathbb{R}_{+}$и некоторой подгруппы $P_{0}$, обладающей однопараметрическим семейством неприводимых унитарных представлений $T_{r}, r>0$, на которых транзитивно действует группа автоморфизмов $\mathbb{R}_{+}$; семейство $T_{r}, r>0$, есть деформация тривиального представления (при $r=0$ ).

Первый факт сводит задачу построения представления группы токов $G^{X}$ к построению представления группы токов $P^{X}$, где $P$ - максимальная параболическая подгруппа группы $G$, последняя задача, в свою очередь, решается благодаря второму факту и, главное, существованию замечательной сигма-конечной меры в пространстве распределений, о которой мы в заключение этого введения коротко скажем несколько слов. Цель настоящей статьи состоит в подробном описании этого решения. Более развернутое изложение всего круга вопросов, относящихся к представлениям групп токов, авторы надеются дать в готовящейся книге.

\footnotetext{
${ }^{1}$ Вся техника, используемая далее, применима к двум бесконечномерным группам $\mathrm{O}(\infty, 1), \mathrm{U}(\infty, 1)$, что позволяет построить желаемое представление соответствующих групп токов.
} 
Мера, названная в [19]-[21] бесконечномерной мерой Лебега и обозначаемая далее через $\mathscr{L}$, появилась в работах [11], [12] как мера, преобразование Лапласа которой естественно возникало при диагонализации представления группы $\mathrm{SL}(2, \mathbb{R})$-токов. Позже выявились замечательные связи и свойства этой меры и однопараметрического семейства $\mathscr{L}_{\theta}, \theta>0$, в которое она включена (при $\theta=1)$. Она связана с мерами Пуассона-Дирихле, гамма-процессом и процессами Леви, отвечающими устойчивым законам. Фундаментальное свойство этой меры - ее инвариантность относительно континуального аналога картановской группы и единственность при естественных предположениях, о чем более подробно написано в работе [21]; см. также [22], где подробно исследовано свойство квазиинвариантности меры Леви гамма-процесса и эквивалентность ee мере $\mathscr{L}_{\theta}$.

В заключение заметим, что до настоящего времени совершенно не исследованы, для целей построения мультипликативного интеграла представлений, возможности других факторизаций, т. е., во-первых, нефоковских факторизаций типа I, существование которых было доказано в работе [23], и, во-вторых, факторизаций типа II и III. Последние возникают в проективных представлениях групп токов на окружности, т. е. в модулях Каца-Муди. Эти представления сильно отличаются от описанных выше, для них существенна положительность энергии, одномерность базы $X$ и проективность. Вполне возможно, что привлечение нефоковских факторизаций расширит класс групп, для которых существует мультипликативный интеграл, и класс самих представлений. Следует также иметь в виду, что могут существовать и неунитарные аналоги этой теории, которые также не изучались.

1.3. Изложим кратко содержание статьи. В разделе 2 дано описание бесконечномерной меры Лебега $\mathscr{L}$ - основы последующей конструкции интегральных моделей представлений групп $G^{X}$ и приведено вычисление интегралов по этой мере.

Разделы 3 и 4 посвящены построению и изучению интегральных моделей групп токов $P^{X}$ для класса локально компактных групп $P$, представимых в виде полупрямого произведения $P=\mathbb{R}_{+}^{*} \wedge P_{0} . \mathrm{K}$ классу таких групп принадлежат, в частности, максимальные параболические подгруппы простых групп Ли ранга 1, т. е. групп $\mathrm{SO}(n, 1), \mathrm{SU}(n, 1)$ и $\mathrm{Sp}(n, 1)$.

Элементы $r \in \mathbb{R}_{+}^{*}$ индуцируют автоморфизмы $g \mapsto g^{r}$ подгруппы $P^{0}$ и тем самым связывают с каждым ее представлением $T$ однопараметрическое семейство представлений операторами $T_{r}(g)=T\left(g^{r}\right)$. Рассматриваются представления $T$ группы $P_{0}$, в пространстве $H$ которых существует вектор $h$ с нормой $\|h\|=1$ такой, что имеет место оценка

$$
\left\|T_{r}(g) h-h\right\|<c(g) r \quad \text { для любого } g \in P_{0}
$$

при достаточно малом $r$. Из этого условия следует, что семейство представлений $T_{r}$ является деформацией тривиального представления группы $P_{0}$, т. е. сходится при $r \rightarrow 0$ к тривиальному в топологии Фелла. Мы называем это свойство каноничностью. Отметим, что оно является более сильным, чем свойство каноничности, введенное в других работах (см., например, [1], [12], [24]). 
Прямой интеграл $\widetilde{T}$ представлений $T_{r}$ по мультипликативной мере $d^{*} r=$ $r^{-1} d r$, т. е.

$$
\int_{0}^{\infty} T_{r} d^{*} r
$$

естественно продолжается до представления $\widetilde{T}$ всей группы $P=\mathbb{R}_{+}^{*} \curlywedge P_{0}$ : $\left(\widetilde{T}\left(r_{0}\right) f\right)(r)=f\left(r_{0} r\right)$ при $r_{0} \in \mathbb{R}_{+}^{*}$. Полученное так представление обладает нетривиальным 1-коциклом.

Конструкция интегральной модели представления группы $P^{X}$ аналогична построению представления $\widetilde{T}$ группы $P$ по представлению $T$ группы $P_{0}$. В этой конструкции группа $P_{0}$ заменяется группой токов $P_{0}^{X}$, а представления $T_{r}$ группы $P_{0}$ - представлениями $T_{\xi}(g(\cdot))=\bigotimes_{k=1}^{\infty} T_{r_{k}}\left(x_{k}\right)$ группы $P_{0}^{X}$ в счетных тензорных степенях пространства $H$. Здесь $\xi$ пробегает точки конуса

$$
l_{+}^{1}(X)=\left\{\xi=\sum_{k=1}^{\infty} r_{k} \delta_{x_{k}} \mid r_{k}>0, \sum_{k} r_{k}<\infty, x_{k} \in X\right\},
$$

на котором сосредоточена бесконечномерная мера Лебега $\mathscr{L}$. Чтобы получить искомое представление группы токов $P^{X}$, мы рассматриваем прямой интеграл по мере $\mathscr{L}$ этих представлений группы $P_{0}^{X}$ и, основываясь на свойствах меры $\mathscr{L}$, строим продолжение полученного так представления группы $P_{0}^{X}$ до представления группы $P^{X}$. Полученное так представление группы $P^{X}$ мы называем интегральной моделью и обозначаем через INT T.

Устанавливается свойство неприводимости получаемых так представлений и их связь с фоковскими представлениями.

Последующие разделы посвящены интегральным моделям представлений групп токов $P^{X}$, где $P$ - максимальные параболические подгруппы групп Ли $\mathrm{O}(n, 1), \mathrm{U}(n, 1)$ и $\mathrm{Sp}(n, 1)$ (разделы $5,7,8$ соответственно). Случай

$$
P \subset \mathrm{SL}(2, \mathbb{R}) \cong \mathrm{SU}(1,1)
$$

выделен в отдельный раздел (раздел 6). Эти группы $P$ представимы в виде полупрямых произведений $P=\mathbb{R}_{+}^{*} \wedge P_{0}$, и их подгруппы $P_{0}$ обладают каноническими представлениями. В каждом из этих случаев дается описание канонических представлений подгруппы $P_{0}$, а тем самым, согласно общей конструкции, и описание отвечающих им интегральных моделей представлений групп токов $P^{X}$.

Главная задача здесь - получить представления групп токов $\mathrm{O}(n, 1)^{X}$ и $\mathrm{U}(n, 1)^{X}$ как продолжения интегральных моделей INT $T$ представлений соответствующих групп токов $P^{X}$. С этой целью рассматриваются такие канонические представления $T$ групп $P_{0}$, что ассоциируемые с ними особые представления группы $P$ продолжаемы до представления соответствующей простой группы Ли. Для них явно строится продолжение интегральной модели INT T представления группы токов до представления группы токов соответствующей простой группы Ли. Полученные модели сравниваются с их фоковскими моделями, построенными в [12], [25]. Попутно в этой конструкции возникают и новые модели особых представлений групп $\mathrm{O}(n, 1)$ и $\mathrm{U}(n, 1)$, представляющие самостоятельный интерес. 


\section{2. Мера $\mathscr{L}$ в пространстве распределений}

2.1. Определение меры $\mathscr{L}$. Рассматривается произвольное многообразие $X$ с фиксированной непрерывной неотрицательной конечной борелевской мерой $m$. Конструкция интегральных моделей представлений групп токов $G^{X}$ основана на существовании в пространстве $D(X)$ распределений Шварца на $X$ меры $\mathscr{L}$, являющейся бесконечномерным аналогом лебеговской меры. Эта мера возникла в [11], [12] и была исследована в цикле работ [19]-[21]. Здесь мы ограничимся лишь ее определением и перечислением основных свойств, используемых далее в статье.

Свяжем с каждым конечным разбиением $X$ на измеримые подмножества,

$$
\alpha: \quad X=\bigcup_{k=1}^{n} X_{k}, \quad m\left(X_{k}\right)=\lambda_{k}, \quad k=1, \ldots, n,
$$

конус $\mathscr{F}_{\alpha}=\mathbb{R}_{+}^{n}$ кусочно постоянных положительных функций вида

$$
f(x)=\sum_{k=1}^{m} f_{k} \chi_{k}(x), \quad f_{k}>0,
$$

где $\chi_{k}$ - характеристическая функция подмножества $X_{k}$, и обозначим через $\Phi_{\alpha}=\left(\mathbb{R}_{+}^{m}\right)^{\prime}$ двойственный ему конус в пространстве распределений.

Определим меру $\mathscr{L}_{\alpha}$ на $\Phi_{\alpha}$ равенством

$$
d \mathscr{L}_{\alpha}\left(x_{1}, \ldots, x_{n}\right)=\prod_{k=1}^{n} \frac{x_{k}^{\lambda_{k}-1} d x_{k}}{\gamma\left(\lambda_{k}\right)}, \quad \text { где } \quad \lambda_{k}=m\left(X_{k}\right) .
$$

Пусть $D_{+}(X) \subset D(X)$ - множество (конус) неотрицательных распределений Шварца на $X$ и $l_{+}^{1}(X) \subset D_{+}(X)$ - подмножество (конус) дискретных конечных (неотрицательных) мер на $X$, т. е.

$$
l_{+}^{1}(X)=\left\{\xi=\sum_{k=1}^{\infty} r_{k} \delta_{x_{k}} \mid r_{k}>0, \sum_{k} r_{k}<\infty, x_{k} \in X\right\} .
$$

Имеет место естественная проекция $D_{+}(X) \rightarrow \Phi_{\alpha}$.

ТЕОРЕМА-ОПРЕДЕЛЕНИЕ. Существует сигма-конечная (бесконечная) меpa $\mathscr{L}$ на конусе $D_{+}(X)$, конечная на любом компакте, сосредоточенная на конусе $l_{+}^{1}(X)$, такая, что для каждого разбиения $\alpha$ пространства $X$ ее проекция на подпространство $\Phi_{a}$ имеет вид (2.1).

Эта мера однозначно определяется своим преобразованием Лапласа

$$
F(f) \equiv \int_{l_{+}^{1}(X)} \exp \left(-\sum_{k} r_{k} f\left(x_{k}\right)\right) d \mathscr{L}(\xi)=\exp \left(-\int_{X} \log f(x) d m(x)\right),
$$

где $f$ - произвольная неотрицательная измеримая функиия на $(X, m)$ с конечным интегралом от логарифма.

Элементы из $l_{+}^{1}(X)$ будем кратко обозначать через $\xi=\left\{r_{k}, x_{k}\right\}_{k=1}^{\infty}$ или, еще короче, через $\xi=\left\{r_{k}, x_{k}\right\}$ (последовательности, различающиеся только порядком членов, считаются отождествленными). 
ЗАмечАниЕ. Преобразование Лапласа корректно определено для широкого класса сигма-конечных мер $\mathscr{L}$, для которых есть достаточное число линейных функционалов, имеющих небесконечное распределение, и имеет место теорема единственности мер с заданным преобразованием Лапласа.

Подобно классическому преобразованию Лапласа, формула (2.2) для характеристического функционала меры $\mathscr{L}$ сохраняет смысл для любой комплекснозначной функции $f(x)$ с положительной вещественной частью и сходящимся интегралом $\int_{X} \log f(x) d m(x)$. Здесь и всюду в дальнейшем через log обозначается ветвь логарифма на комплексной плоскости с разрезом вдоль вещественной отрицательной полуоси, удовлетворяющая условию $\log 1=0$.

Для нас будет важно следующее характеристическое определение меры $\mathscr{L}$. Обозначим через $A(X)$ группу (по умножению) всех неотрицательных измеримых функций $a(x)$ на $X$ со сходящимся интегралом $\int_{X} \log a(x) d m(x)=c$ и через $A_{0}(X)$ подгруппу функций $a(x)$, для которых $c=0$. Поставим в соответствие каждой функции $a \in A(x)$ оператор $M_{a}$ умножения элементов $\xi=$ $\sum_{k} r_{k} \delta_{x_{k}}$ конуса $l_{+}^{1}(X)$ на функцию $a(x)$ :

$$
M_{a} \sum_{k} r_{k} \delta_{x_{k}}=\sum_{k} a\left(x_{k}\right) r_{k} \delta_{x_{k}} .
$$

Теорема 2.1. Мера $\mathscr{L}$ на конусе $l_{+}^{1}(X)$ полностъю характеризуется следующими двумя свойствами.

1) Проективная инвариантность относительно группы $\mathscr{M}$ мультипликаторов $M_{a}:$ для любой функиии $a \in A(X)$ оператор $M_{a}$ умножает меру $\mathscr{L}$ на $\exp c$, m.e.

$$
d \mathscr{L}(a(\cdot) \xi)=\exp \left(\int_{X} \log a(x) d m(x)\right) d \mathscr{L}(\xi) .
$$

$B$ частности, мера $\mathscr{L}$ инвариантна относительно подгруппъ $\mathscr{M}_{0}$ мультипликаторов $M_{a}$, где а $\in A_{0}(X)$.

2) Инвариантность и эргодичность относительно группь всех преобразований $(X, m)$, сохраняющих меру. ${ }^{2}$

Выполнение этих свойств для меры $\mathscr{L}$ вытекает из формулы $(2.2)$ для преобразования Лапласа этой меры. Достаточность см. в [20], [21].

Из теоремы 2.1 следует, что определенные так меры $\mathscr{L}$ зависят только от одного параметра $\theta=m(X)$ и что при свертке они образуют мультипликативную полугруппу относительно $\theta>0$.

В конструкции интегральной модели достаточно ограничиться только одной из этих мер, а потому в дальнейшем будем предполагать, что $\theta=1$, т. е. $m-$ вероятностная мера. Меру $\mathscr{L}$ естественно назвать бесконечномерной лебеговой мерой, поскольку она обобщает свойства инвариантности конечномерной лебеговой меры на неотрицательном октанте. Новым в бесконечномерном случае является ее эргодичность относительно группы мультипликаторов, которую выделим особо.

\footnotetext{
${ }^{2}$ Как показано в [20], утверждение 2) является следствием из 1).
} 
Теорема 2.2. Группа мультипликаторов $\mathscr{M}$ действует эргодично на конусе $l_{+}^{1}(X)$ с мерой $\mathscr{L}{ }^{3}$

2.2. Вычисление некоторых интегралов по мере $\mathscr{L}$. Применим свойства меры $\mathscr{L}$ к вычислению следующего интеграла по мере $\mathscr{L}$ :

$$
I=\int_{l_{+}^{1}(X)}\left(\prod_{k=1}^{\infty} \varphi\left(r_{k}, x_{k}\right)\right) d \mathscr{L}(\xi)
$$

где $\varphi(r, x)$ - функция на $\mathbb{R}_{+}^{*} \times X$, удовлетворяющая условиям

$$
\varphi(0, x) \equiv 1 \quad \text { и } \quad \int_{X} \int_{0}^{\infty}\left(\varphi(r, x)-e^{-r}\right) r^{-1} d r d m(x)<\infty .
$$

ТеОрема 2.3. Имеет место равенство:

$$
\int_{l_{+}^{1}(X)}\left(\prod_{k=1}^{\infty} \varphi\left(r_{k}, x_{k}\right)\right) d \mathscr{L}(\xi)=\exp \left(\int_{X} \int_{0}^{\infty}\left(\varphi(r, x)-e^{-r}\right) r^{-1} d r d m(x)\right) .
$$

ДокАЗАтельство. При проектировании $D_{+}(X) \rightarrow \Phi_{\alpha}$, где, напоминаем, $\Phi_{\alpha}$ - конечномерное пространство, ассоциированное с разбиением $\alpha: X=$ $\bigcup_{k=1}^{n} X_{k}$, левая часть равенства (2.4) принимает вид:

$$
I_{\alpha}=\prod_{k=1}^{m} I_{\alpha}^{k}, \quad I_{\alpha}^{k}=\frac{1}{\Gamma\left(\lambda_{k}\right)} \int_{0}^{\infty} \varphi_{\alpha, k}\left(r_{k}\right) r_{k}^{\lambda_{k}-1} d r_{k},
$$

где $\lambda_{k}=m\left(X_{k}\right)$ и $\varphi_{\alpha, k}\left(r_{k}\right)=\lambda_{k}^{-1} \int_{X_{k}} \varphi\left(r_{k}, x\right) d m(x)$. Исходный интеграл $I$ является индуктивным пределом интегралов $I_{\alpha}$ по множеству разбиений $\alpha$.

Поскольку $\frac{1}{\Gamma\left(\lambda_{k}\right)} \int_{0}^{\infty} e^{-t} r_{k}^{\lambda_{k}-1} d r_{k}=1$, интеграл $I_{\alpha}^{k}$ можно представить в виде

$$
I_{\alpha}^{k}=1+\frac{1}{\Gamma\left(\lambda_{k}\right)} \int_{0}^{\infty}\left(\varphi_{\alpha, k}\left(r_{k}\right)-e^{-r_{k}}\right) r_{k}^{\lambda_{k}-1} d r_{k}
$$

Отсюда следует, что

$$
I_{\alpha}^{k}=1+\lambda_{k} \int_{0}^{\infty}\left(\varphi_{\alpha, k}\left(r_{k}\right)-e^{-r_{k}}\right) r_{k}^{-1} d r_{k}+O\left(\lambda_{k}^{2}\right),
$$

а потому

$$
I_{\alpha}^{k}=\exp \left(\lambda_{k} \int_{0}^{\infty}\left(\varphi_{\alpha, k}\left(r_{k}\right)-e^{-r_{k}}\right) r_{k}^{-1} d r_{k}\right)+O\left(\lambda_{k}^{2}\right) .
$$

Таким образом, с точностью до членов порядка выше 1 относительно $\lambda_{k}$ получаем:

$$
I_{\alpha} \cong \exp \left(\sum_{k=1}^{m} \lambda_{k} \int_{0}^{\infty}\left(\varphi_{\alpha, k}(r)-e^{-r}\right) r^{-1} d r\right) .
$$

\footnotetext{
${ }^{3} \mathrm{~B}$ конечномерном случае эта группа есть SDiag + (положительная часть картановской группы), действующая транзитивно на каждой из гиперсфер $x_{1} x_{2} \cdots x_{n}=$ const, $x_{i}>0$, $i=1,2, \ldots, n$, т. е. действие SDiag + на конусе $\mathbb{R}_{+}^{n}$ не эргодично.
} 
Поскольку $\sum_{k=1}^{m}\left(\lambda_{k}\left(\varphi_{\alpha, k}(r)-e^{-r}\right)\right)=\int_{X}\left(\varphi(r, x)-e^{-r}\right) d m(x)$, полученное выражение можно представить в виде

$$
I_{\alpha} \cong \exp \left(\int_{X} \int_{0}^{\infty}\left(\varphi(r, x)-e^{-r}\right) r^{-1} d r d m(x)\right) .
$$

Отсюда, перейдя к индуктивному пределу по множеству разбиений $\alpha$, получаем теорему ${ }^{4}$.

СледСТвиЕ. Если $\varphi(r, x)=\sum_{i=1}^{n} c_{i} \varphi_{i}(r, x)$, әде $c_{i}>0, \sum c_{i}=1$ и функиии $\varphi_{i}(r, x)$ удовлетворяют условию $(2.5)$, то

$$
\int_{l_{+}^{1}(X)}\left(\prod_{k=1}^{\infty} \varphi\left(r_{k}, x_{k}\right)\right) d \mathscr{L}(\xi)=\prod_{i=1}^{n} \exp \left(c_{i} \int_{X} \int_{0}^{\infty}\left(\varphi_{i}(r, x)-e^{-r}\right) r^{-1} d r d m(x)\right) .
$$

ПримеР. Пусть $\varphi(r, x)=e^{-r^{\sigma} a(x)}$, где $\sigma \geqslant 1$ и $\operatorname{Re} a(x)>0$. В этом случае получаем:

$$
\int_{l_{+}^{1}(X)}\left(\prod_{k=1}^{\infty} e^{-r_{k}^{\sigma} a\left(x_{k}\right)}\right) d \mathscr{L}(\xi)=\exp \left(\int_{X} \int_{0}^{\infty}\left(e^{-r^{\sigma} a(x)}-e^{-r}\right) r^{-1} d r d m(x)\right) .
$$

Вычислим интеграл по $r$. Имеем:

$$
\begin{aligned}
\int_{0}^{\infty}\left(e^{-r^{\sigma} a(x)}-e^{-r}\right) r^{-1} d r & =\lim _{\lambda \rightarrow 0}\left(\int_{0}^{\infty}\left(e^{-r^{\sigma} a(x)}-e^{-r}\right) r^{\lambda-1} d r\right) \\
& =\lim _{\lambda \rightarrow 0}\left(\sigma^{-1} \Gamma\left(\frac{\lambda}{\sigma}\right) a^{-\lambda / \sigma}(x)-\Gamma(\lambda)\right) .
\end{aligned}
$$

Отсюда, поскольку $\Gamma(\lambda) \sim \lambda^{-1}+\gamma$ при $\lambda \rightarrow 0$, где $\gamma-$ постоянная Эйлера, следует:

$$
\int_{0}^{\infty}\left(\exp \left(-r^{\sigma} a(x)\right)-\exp (-r)\right) r^{-1} d r=-\sigma^{-1} \log a(x)+\left(\sigma^{-1}-1\right) \gamma .
$$

Значит,

$$
\begin{aligned}
\int_{l_{+}^{1}(X)} & \left(\prod_{k=1}^{\infty} \exp \left(-r_{k}^{\sigma} a\left(x_{k}\right)\right)\right) d \mathscr{L}(\xi) \\
= & \exp \left(\left(\sigma^{-1}-1\right) \gamma\right) \exp \left(-\sigma^{-1} \int_{X} \log a(x) d m(x)\right)
\end{aligned}
$$

В частности, при $\sigma=1$ получаем исходную формулу для преобразования Лапласа меры $\mathscr{L}$ :

$$
\int_{l_{+}^{1}(X)} \prod_{k=1}^{\infty} \exp \left(-\sum r_{k} a\left(x_{k}\right)\right) d \mathscr{L}(\xi)=\exp \left(-\int_{X} \log a(x) d m(x)\right) .
$$

\footnotetext{
${ }^{4}$ Поскольку мера $\mathscr{L}$ абсолютно непрерывна по мере, порождаемой гамма-процессом Леви (см. [22]), то приведенный результат может быть получен с помощью аналогичных вычислений для процессов Леви.
} 


\section{3. Канонические представления группы $P_{0}$ и ассоциированные с ними представления группы токов $P_{0}^{X}$}

3.1. Определение канонических представлений. Рассматриваются полупрямые произведения $P=A \wedge P_{0}$ локально компактной группы $P_{0}$ и ее мультипликативной группы автоморфизмов $A \cong \mathbb{R}_{+}^{*}$. Будем обозначать через $g^{a}$ образ элемента $g \in P_{0}$ под действием автоморфизма $a \in A$.

$B$ дальнейшем термин "представление" подразумевает, если не оговорено противное, ортогональное или унитарное представление группы.

Группа автоморфизмов $A$ естественно действует в множестве всех представлений группы $P_{0}$, преобразуя каждое представление $T(g)$ в представление $T_{a}(g)=T\left(g^{a}\right)$.

ОПРеДЕЛЕНИЕ 1 . Назовем представление $T$ группы $P_{0}$ в гильбертовом пространстве $H$ каноническим, если существуют циклический вектор $h \in H$ с нормой $\|h\|=1$ и изоморфизм $\sigma: \mathbb{R}_{+}^{*} \rightarrow A$ такие, что

1) имеет место оценка

$$
\left\|T_{\sigma(r)}(g) h-h\right\|<c(g) r \quad \text { для любого } g \in P_{0}
$$

при достаточно малом $r$;

2) представления $T_{a}(g)=T\left(g^{a}\right)$ группы $P_{0}$ попарно не эквивалентны.

Назовем циклический вектор $h \in H$, удовлетворяющий условию (3.1), почти инвариантным относительно представления $T$, а представления $T_{a}(g)=T\left(g^{a}\right)$ группы $P_{0}$ - сопряженными с представлением $T$.

В дальнейшем будем отождествлять элементы $a \in A$ с их прообразами $r \in \mathbb{R}_{+}^{*}$ при изоморфизме $\sigma$ и писать $g^{r}$ и $T_{r}$ вместо $g^{\sigma(r)}$ и $T_{\sigma(r)}$. Таким образом, условие (3.1) будет записываться в виде

$$
\left\|T_{r}(g) h-h\right\|<c(g) r \quad \text { для любого } g \in P_{0} .
$$

Из определения 1 следует, что представления $T_{r}$ образуют деформацию единичного представления группы $P_{0}$, т. е. в топологии Фелла на множестве представлений любая окрестность единичного представления содержит все представления $T_{r}$, начиная с достаточно малого $r$.

Из определения каноничности также вытекает следующее утверждение.

ПРеДЛОЖЕНИЕ 3.1. Если представление $T$ группљ $P_{0}$ в пространстве $H$ канонично, то для любой суммируемой числовой последовательности $\left\{r_{k}\right\}$, $r_{k}>0\left(\sum r_{k}<\infty\right)$, и любого $g \in P_{0}$ имеем:

$$
\sum_{k=1}^{\infty}\left\|T_{r_{k}}(g) h-h\right\|<\infty,
$$

где $h \in H$ - почти инвариантный относительно $T$ вектор.

Заметим, что в пространстве $H$ канонического представления $T$ почти инвариантный вектор $h$ может быть не единственным; пример (одномерное расширение группы Гейзенберга) будет рассмотрен позже.

Для дальнейшего полезно следующее утверждение. 
ПрЕДЛОЖЕНИЕ 3.2. Если в пространстве $H$ представления Т группы $P_{0}$ существует единственный, с точностъю до множителя, единичный циклический вектор $h$, удовлетворяющий условию 1) определения 1, то представление Т канонично, т.е. оно удовлетворяет также условию 2) этого определения.

ДокАЗАтельство. Предположим, что условие 2) не выполнено, т. е. существуют два эквивалентных представления $T_{r}$, для определенности, представления $T=T_{1}$ и $T_{r_{0}}$, где $r_{0}<1$. Значит, существует унитарный оператор $A$ такой, что $A^{-1} T(g) A=T_{r_{0}}(g)$ для любого $g \in P_{0}$. Тогда имеем:

$$
A^{-n} T_{r}(g) A^{n}=T_{r_{0}^{n} r}(g) \quad \text { для любых } g \in P_{0}, r>0 \text { и } n=1,2, \ldots
$$

Следовательно, если $h \in H$ - циклический единичный вектор, удовлетворяющий условию 1), то из этого условия следует:

$$
\left\|T_{r}(g) A^{n} h-A^{n} h\right\|<c(g) r_{0}^{n} r \quad \text { при любом } g \in P_{0} .
$$

В силу этой оценки, все векторы $h_{n}=A^{n} h$ также удовлетворяют условию 1$)$. При этом $A h \neq c h$. В противном случае можно было бы предположить, что $A h=h$, а тогда из полученной оценки следовало бы, что

$$
\left\|T_{r}(g) h-h\right\|<c(g) r_{0}^{n} r \quad \text { при любом } g \in P_{0} \text { и } n=1,2, \ldots,
$$

т. е. $h$ - инвариантный вектор, что противоречит предположению о его цикличности. Таким образом, вектор $h$, удовлетворяющий условию 1$)$, не единствен, что противоречит условию предложения.

ПримечАниЕ. В ряде предшествующих статей (см., например, [1], [12], [24]) под термином "канонические представления" подразумевалось однопараметрическое семейство представлений $T_{r}, r>0$, со сферическими функциями вида $\varphi_{r}(g)=e^{r \psi(g)}$.

Более слабым условием каноничности семейства представлений $T_{r}$ является существование производной при $r \rightarrow 0$ у сферических функций этого семейства,

$$
\psi(g)=\left.\frac{d \varphi_{r}(g)}{d r}\right|_{r=0},
$$

являющейся условно положительно определенной функцией (генератор системы). Этому условию удовлетворяет, например, семейство представлений дополнительной серии групп $\mathrm{SO}(n, 1)$ и $\mathrm{SU}(n, 1)$. Существование у них генератора следует из оценки $\left\|T_{r}(g) h-h\right\|^{2}<c(g) r$, более слабой, чем условия $(3.2)$ и (3.3). Различные подходы к понятию каноничности мы обсудим в другом месте ${ }^{5}$.

3.2. Представления группы $l^{\infty}\left(P_{0}\right)$ и группы токов $P_{0}^{X}$, ассоциированные с каноническими представлениями подгруппы $P_{0}$. Обозначим через $l^{\infty}\left(P_{0}\right)$ группу всех бесконечных ограниченных последовательностей $g=\left\{g_{1}, g_{2}, \ldots\right\}$ элементов из $P_{0}$ с покоординатным умножением и поставим

\footnotetext{
${ }^{5}$ Канонические представления в близком смысле рассматривались также в других работах, см., например, [26].
} 
в соответствие каждому каноническому представлению $T$ группы $P_{0}$ семейство представлений группы $l^{\infty}\left(P_{0}\right)$.

Для этого воспользуемся следующим хорошо известным определением.

ОПРЕДЕЛЕНИЕ 2. Счетной тензорной степенью гильбертова пространства $H$ со стабилизирующим единичным вектором $h \in H$ называется пополнение индуктивного предела конечных тензорных степеней $\bigotimes_{i=1}^{n} H$ пространства $H$ при их изометрическом вложении: $\bigotimes_{i=1}^{n} H \ni f \mapsto f \otimes h \in \bigotimes_{i=1}^{n+1} H$. Будем обозначать этот предел через $\widetilde{H}=\bigotimes_{i=1}^{\infty}(H, h)$ или, коротко, через $\bigotimes_{i=1}^{\infty} H$.

Другими словами, $\widetilde{H}$ есть гильбертово пространство, полученное пополнением по норме пространства $\lim \operatorname{ind}_{n \rightarrow \infty} \bigotimes_{i=1}^{n} H$.

Элементы $\bigotimes_{k=1}^{n} f_{k}$ из подпространств $\bigotimes_{k=1}^{n} H \subset \widetilde{H}$ естественно представлять в форме бесконечных произведений, стабилизирующихся на $(n+1)$-м шаге:

$$
y_{n}=f_{1} \otimes f_{2} \otimes \cdots \otimes f_{n} \otimes h \otimes h \otimes \cdots, \quad \text { где } f_{k} \in H
$$

они образуют тотальное подмножество в $\widetilde{H}$.

Лемма. Если $\sum_{n=1}^{\infty}\left\|f_{n}-h\right\|<\infty$, то последовательность $\left\{y_{n}\right\}$ вида (3.5) сходится по норме пространства $\widetilde{H}$.

Пределы таких последовательностей $\left\{y_{n}\right\}$ будем записывать в форме бесконечных произведений $y=\bigotimes_{n=1}^{\infty} f_{n}$, где $\sum_{n=1}^{\infty}\left\|f_{n}-h\right\|<\infty$, и в дальнейшем при описании пространства $\widetilde{H}$ будем ограничиваться только такими элементами из $\widetilde{H}$ и их конечными линейными комбинациями.

ОПРЕДЕЛЕНиЕ 3. Свяжем с каждым каноническим представлением $T$ группы $P_{0}$ в пространстве $H$ с почти инвариантным вектором $h \in H$ и с каждой последовательностью $\left\{r_{k}\right\}, r_{k}>0$, такой, что $\sum r_{k}<\infty$, следующее представление группы $l^{\infty}\left(P_{0}\right)$ в пространстве $\bigotimes_{k=1}^{\infty}(H, h)$ :

$$
\widetilde{T}_{\left\{r_{n}\right\}}(g)\left(\bigotimes_{n=1}^{\infty} f_{n}\right)=\bigotimes_{n=1}^{\infty}\left(T_{r_{n}}\left(g_{n}\right) f_{n}\right) \quad \text { при } g=\left\{g_{1}, g_{2}, \ldots\right\} .
$$

Убедимся, что это определение корректно, т. е. из условия $\sum_{n=1}^{\infty}\left\|f_{n}-h\right\|<\infty$ следует, что $\sum_{n=1}^{\infty}\left\|T_{r_{n}}\left(g_{n}\right) f_{n}-h\right\|<\infty$ для любого $g=\left\{g_{1}, g_{2}, \ldots\right\} \in l^{\infty}\left(P_{0}\right)$. В самом деле, имеем:

$$
\left\|T_{r_{n}}\left(g_{n}\right) f_{n}-h\right\| \leqslant\left\|f_{n}-h\right\|+\left\|T_{r_{n}}\left(g_{n}\right) h-h\right\| .
$$

Из каноничности представления $T$ и ограниченности последовательности $\left\{g_{n}\right\}$ следует, что для любого $g \in l^{\infty}\left(P_{0}\right)$ верна оценка $\left\|T_{r_{n}}\left(g_{n}\right) h-h\right\| \leqslant c r_{n}$. Значит, из условия $\sum r_{k}<\infty$ следует, что $\sum_{n=1}^{\infty}\left\|T_{r_{n}}\left(g_{n}\right) h-h\right\|<\infty$. Отсюда следует утверждение.

ПРЕДЛОЖЕНИЕ 3.3. Если каноническое представление $T$ группы $P_{0}$ неприводимо, то ассоциированные с $T$ представления $T_{\left\{r_{n}\right\}}$ группы $l^{\infty}\left(P_{0}\right)$ неприводимы и попарно не эквивалентны. 
В самом деле, неприводимость представлений $T_{\left\{r_{n}\right\}}$ группы $l^{\infty}\left(P_{0}\right)$ следует непосредственно из неприводимости представления $T$ группы $P_{0}$. Их попарная неэквивалентность следует из попарной неэквивалентности представлений $T_{r}$ группы $P_{0}$, сопряженных с представлением $T$.

Построим теперь, исходя из представлений $T_{\left\{r_{n}\right\}}$ группы $l^{\infty}\left(P_{0}\right)$, представления группы токов $P_{0}^{X}$. Для этого поставим в соответствие каждому элементу $\xi=\left\{r_{k}, x_{k}\right\} \in l^{1}(X)$ гомоморфизм $P_{0}^{X} \rightarrow l^{\infty}\left(P_{0}\right)$ :

$$
\sigma_{\xi}: g(\cdot) \mapsto\left(g\left(x_{1}\right), g\left(x_{2}\right), \ldots\right) .
$$

Тем самым для каждого элемента $\xi=\left\{r_{k}, x_{k}\right\} \in l^{1}(X)$ определено представление $T_{\xi}$ группы $P_{0}^{X}$, пропущенное через этот гомоморфизм. Пространство этого представления есть счетное тензорное произведение $H_{\xi}=\bigotimes_{k=1}^{\infty} H_{r_{k}}, H_{r_{k}}=H$. Операторы представления $T_{\xi}, \xi=\left\{r_{k}, x_{k}\right\}$, в пространстве $H_{\xi}$ задаются формулой:

$$
T_{\xi}(g(\cdot))\left(\bigotimes_{k=1}^{\infty} f_{k}\right)=\bigotimes_{k=1}^{\infty}\left(T_{r_{k}}\left(g\left(x_{k}\right)\right) f_{k}\right) .
$$

Отметим, что представление $T_{\xi}, \xi=\left\{r_{n}, x_{n}\right\}$, группы $P_{0}^{X}$ есть счетное тензорное произведение локальных представлений группы $P_{0}^{X}$ :

$$
T_{\xi}=\bigotimes_{n=1}^{\infty} T_{r_{n}, x_{n}}, \quad \text { где } T_{r_{n}, x_{n}}(g(\cdot))=T_{r_{n}}\left(g\left(x_{n}\right)\right) .
$$

ПреДЛОЖЕНИЕ 3.4. Если каноническое представление $T$ группљ $P_{0}$ неприводимо, то представления $T_{\xi}$ группь $P_{0}^{X}$ неприводимы и попарно не эквивалентны.

ДокАЗАтельство. Достаточно убедиться, что представления $T_{r, x}$ и $T_{r^{\prime}, x^{\prime}}$ группы $P_{0}^{X}$ не эквивалентны при $(r, x) \neq\left(r^{\prime}, x^{\prime}\right)$. При $r \neq r^{\prime}$ это следует из попарной неэквивалентности представлений $T_{r}$ группы $P_{0}$. При $r=r^{\prime}$ это следует из разделяемости точек $x$ и $x^{\prime}$ элементами из $P_{0}^{X}$, т. е. существования элемента $g \in P_{0}^{X}$ такого, что $g(x) \neq g\left(x^{\prime}\right)$.

ЗАмечание. Как уже отмечалось, почти инвариантный вектор $h \in H$, acсоциированный с каноническим представлением $T$ группы $P_{0}$, может быть неединственным, например, если $P_{0}$ - группа Гейзенберга (см. ниже). Тогда построенные представления $T_{\xi}$ группы $P_{0}^{X}$ зависят и от выбора почти инвариантного вектора $h \in H$. Нетрудно убедиться, что семейства этих представлений, связанные с почти инвариантными векторами $h$ и $h^{\prime}$, эквивалентны тогда и только тогда, когда $h^{\prime}=c h$, где $|c|=1$.

3.3. Пример: $P=\mathbb{R}_{+}^{*} \curlywedge P_{0}$, где $P_{0}$ - группа Гейзенберга порядка $2 n-1$. Реализуем $P_{0}$ как группу пар $(t, z), t \in \mathbb{R}, z \in \mathbb{C}^{n-1}$, с законом умножения $\left(t_{1}, z_{1}\right)\left(t_{2}, z_{2}\right)=\left(t_{1}+t_{2}-\operatorname{Im}\left(z_{1} z_{2}^{*}\right), z_{1}+z_{2}\right)$. Элементы $r \in \mathbb{R}_{+}^{*}$ и $(t, z) \in P_{0}$ связаны соотношением $r(t, z) r^{-1}=\left(r^{2} t, r z\right)$.

С точностью до сопряженности, имеются два бесконечномерных унитарных неприводимых канонических представления группы $P_{0}$ (см. [27]), действующих 
в гильбертовых пространствах $H^{ \pm}$соответственно целых аналитических и целых антианалитических функций $f(z)=f\left(z_{1}, \ldots, z_{n-1}\right)$ на $\mathbb{C}^{n-1}$ с нормой

$$
\|f\|^{2}=\int_{\mathbb{C}^{n-1}}|f(z)|^{2} \exp \left(-z z^{*}\right) d \mu(z)
$$

где $z z^{*}=\sum z_{i} \bar{z}_{i}$ и $d \mu(z)$ - лебегова мера на $\mathbb{C}^{n-1}$, нормированная условием

$$
\int_{\mathbb{C}^{n-1}} \exp \left(-z z^{*}\right) d \mu(z)=1 .
$$

Операторы представления $T^{+}$в пространств $H^{+}$имеют вид:

$$
\left(T^{+}\left(t_{0}, z_{0}\right) f\right)(z)=\exp \left(\zeta_{0}-z z_{0}^{*}\right) f\left(z+z_{0}\right), \quad \text { где } \zeta_{0}=i t_{0}-\frac{1}{2} z_{0} z_{0}^{*} .
$$

Операторы $T^{-}$второго представления получаются из них комплексным сопряжением.

В этом примере операторы $T_{r}^{+}$представлений, сопряженных с представлением $T^{+}$, задаются формулой

$$
\left(T_{r}^{+}\left(t_{0}, z_{0}\right) f\right)(z)=\exp \left(r^{2} \zeta_{0}-r z z_{0}^{*}\right) f\left(z+r z_{0}\right) .
$$

Нетрудно убедиться, что представления $T^{r}$ попарно не эквивалентны. Далее, из определения нормы в $H^{+}$следует, что любой моном $f(z)=z_{1}^{k_{1}} \cdots z_{n-1}^{k_{n-1}}$, а значит, и любая конечная линейная комбинация таких мономов почти инвариантны относительно представления $T^{+}$, т. е. имеет место оценка $\left\|T_{r}^{+}(g) f-f\right\|<$ $c(g) r$. Следовательно, представление $T^{+}$группы Гейзенберга канонично, и множество связанных с ним почти инвариантных векторов всюду плотно в пространстве представления. Аналогичное утверждение справедливо для второго представления $T^{-}$.

4. Представления группы $P=\mathbb{R}_{+}^{*} \curlywedge P_{0}$ и ее группы токов $P^{X}$, ассоциированные с каноническими представлениями подгруппы $P_{0}$

4.1. Представления группы $P=\mathbb{R}_{+}^{*} \curlywedge P_{0}$, ассоциированные с представлениями подгруппы $P_{0}$. Свяжем с каждым ортогональным или унитарным представлением $T$ подгруппы $P_{0}$ прямой интеграл по мультипликативной мере Хаара $d^{*} r=r^{-1} d r$ на $\mathbb{R}_{+}^{*}$ ее представлений $T_{r}$ в пространствах $H_{r}=H$. Определенное так представление $\widetilde{T}$ группы $P_{0}$ действует в гильбертовом пространстве

$$
\mathscr{H}=\int_{0}^{\infty} H_{r} d^{*} r,
$$

т. е. в пространстве сечений $f(r)$ расслоения над $\mathbb{R}_{+}^{*}$, где слоем над $r \in \mathbb{R}_{+}^{*}$ служит пространство $H_{r}$, таких, что $\int_{X}\|f(r)\|^{2} d^{*} r<\infty$. Операторы $\widetilde{T}\left(g_{0}\right)$, $g_{0} \in P_{0}$, действуют на $\mathscr{H}$ послойно, т. е.

$$
\left(\widetilde{T}\left(g_{0}\right) f\right)(r)=T_{r}\left(g_{0}\right) f(r) \quad \text { при } g \in P_{0} .
$$


Это представление подгруппы $P_{0}$ продолжаемо на всю группу $P$. Именно, операторы подгруппы $\mathbb{R}_{+}^{*}$ в пространстве $\mathscr{H}$ задаются равенством

$$
\left(\widetilde{T}\left(r_{0}\right) f\right)(r)=f\left(r_{0} r\right) \quad \text { при } r_{0} \in \mathbb{R}_{+}^{*} .
$$

(Другими словами, операторы $\widetilde{T}\left(r_{0}\right)$ переставляют слои расслоения над $\mathbb{R}_{+}^{*}$.) Очевидно, что операторы $\widetilde{T}\left(r_{0}\right)$ сохраняют скалярное произведение на $\mathscr{H}$, и легко убедиться, что вместе с операторами подгруппы $P_{0}$ они порождают представление всей группы $P$. Назовем это представление группы $P$ ассоииированным с исходным представлением $T$ подгруппы $P_{0}$.

Элементы $g \in P$ будем дальше представлять в форме произведений $g=r g_{0}$, где $r \in \mathbb{R}_{+}^{*}$ и $g_{0} \in P_{0}$.

ПредлОжЕНИЕ 4.1. Если представление Т подгруппы $P_{0}$ канонично и неприводимо, то ассоциированное с $T$ представление $\widetilde{T}$ группы $P$ также неприводимо.

Утверждение следует из неприводимости и попарной неэквивалентности представлений $T_{r}$.

ТеОрема 4.1. Если представление $T$ подгруппы $P_{0}$ в пространстве $H$ канонично, то ассоциированное с $T$ представление $\widetilde{T}$ группы $P$ в пространстве $\mathscr{H}$ обладает нетривиальным 1-коциклом $b: P \rightarrow \mathscr{H}$ вида ${ }^{6}$

$$
b(g, r)=\left(\widetilde{T}(g) f_{0}\right)(r)-f_{0}(r), \quad \text { əде } f_{0}(r)=e^{-r / 2} h_{r},
$$

$h_{r}=h$ - почти инвариантный относительно $T$ вектор в $H$.

В самом деле, из условия каноничности $T$ следует, что $b(g) \in \mathscr{H}$ при любом $g \in P$. Очевидно, далее, что $b$ есть 1 -коцикл. Поскольку $f_{0} \neq \mathscr{H}$, этот 1 -коцикл нетривиален.

Свяжем с 1-коциклом $b(g)$ следующую функцию на $P$ :

$$
c(g)=\left\langle b(g), f_{0}\right\rangle .
$$

ПРЕДЛОЖЕНИЕ 4.2. Имеют место соотношения:

$$
\|b(g)\|^{2}=-\tau(g)-2 \operatorname{Re} c(g) \quad \text { для любого } g \in P,
$$

¿əe

$$
\tau(g)=\log r_{0} \quad \text { npu } g=r_{0} g_{0}
$$

u

$$
\left\langle\widetilde{T}(g) b\left(g^{\prime}\right), b(g)\right\rangle=-c\left(g g^{\prime}\right)+c(g)+c\left(g^{\prime}\right) \quad \text { для любых } g, g^{\prime} \in P .
$$

ДокАЗАТЕЛЬСтво. 1) Из (4.3) следует, что

$$
\|b(g)\|^{2}=\int_{0}^{\infty} F(r) r^{-1} d r
$$

где

$$
F(r)=\left\|\left(\widetilde{T}(g) f_{0}\right)(r)-f_{0}(r)\right\|_{H_{r}}^{2} .
$$

\footnotetext{
${ }^{6} \mathrm{O}$ коциклах со значениями в унитарных представлениях см. [28], [4], [29], а также [7].
} 
Выражение для $F(r)$ преобразуемо к следующему виду:

$$
F(r)=-2 \operatorname{Re}\left\langle\left(\widetilde{T}(g) f_{0}\right)(r)-f_{0}(r), f_{0}(r)\right\rangle_{H_{r}}+\left\|\left(\widetilde{T}(g) f_{0}\right)(r)\right\|_{H_{r}}^{2}-\left\|f_{0}(r)\right\|_{H_{r}}^{2} .
$$

Следовательно, поскольку $\left\|f_{0}(r)\right\|_{H_{r}}^{2}=e^{-r}$ и $\left\|\left(\widetilde{T}(g) f_{0}\right)(r)\right\|_{H_{r}}^{2}=e^{-r_{0} r}$ при $g=$ $r_{0} g_{0}$, получаем:

$$
\|b(g)\|^{2}=-2 \operatorname{Re}\left\langle b(g), f_{0}\right\rangle+\int_{0}^{\infty}\left(e^{-r_{0} r}-e^{-r}\right) r^{-1} d r .
$$

Так как

$$
\begin{aligned}
\int_{0}^{\infty}\left(e^{-r_{0} r}-e^{-r}\right) r^{-1} d r & =\lim _{\lambda \rightarrow 0} \int_{0}^{\infty}\left(e^{-r_{0} r}-e^{-r}\right) r^{\lambda-1} d r \\
& =\lim _{\lambda \rightarrow 0}\left(r_{0}^{-\lambda}-1\right) \Gamma(\lambda)=-\log r_{0}
\end{aligned}
$$

отсюда следует (4.5).

2) Из равенств $b(g)=\widetilde{T}(g) f_{0}-f_{0}$ и $\widetilde{T}(g) b\left(g^{\prime}\right)=b\left(g g^{\prime}\right)-b(g)$ следует:

$$
\left\langle\widetilde{T}(g) b\left(g^{\prime}\right), b(g)\right\rangle=\left\langle\widetilde{T}(g) b\left(g^{\prime}\right), \widetilde{T}(g) f_{0}\right\rangle-c\left(g g^{\prime}\right)+c(g) .
$$

Для доказательства (4.7) остается убедиться, что

$$
\left\langle\widetilde{T}(g) b\left(g^{\prime}\right), \widetilde{T}(g) f_{0}\right\rangle=\left\langle b\left(g^{\prime}\right), f_{0}\right\rangle=c\left(g^{\prime}\right) .
$$

Имеем при $g=r_{0} g_{0}, g^{\prime}=r_{0}^{\prime} g_{0}^{\prime}$ :

$$
\begin{aligned}
\left.\widetilde{T}(g) b\left(g^{\prime}\right)\right|_{H_{r}} & =\exp \left(-\frac{1}{2} r_{0} r_{0}^{\prime} r\right) T_{r_{0} r}\left(g_{0}\right) T_{r_{0} r_{0}^{\prime} r}\left(g_{0}^{\prime}\right) h_{r}-\exp \left(-\frac{1}{2} r_{0} r\right) T_{r_{0} r}\left(g_{0}\right) h_{r}, \\
\left.\widetilde{T}(g) f_{0}\right|_{H_{r}} & =\exp \left(-\frac{1}{2} r_{0} r\right) T_{r_{0} r}\left(g_{0}\right) h_{r} .
\end{aligned}
$$

В силу унитарности операторов $T(g)$ группы $P_{0}$ и мультипликативной инвариантности меры $d^{*} r$ отсюда следует, что

$$
\left\langle\widetilde{T}(g) b\left(g^{\prime}\right), \widetilde{T}(g) f_{0}\right\rangle=\int_{0}^{\infty}\left\langle e^{-r_{0}^{\prime} r / 2} T\left(r_{0}^{\prime} r\right) h-e^{-r / 2} h, e^{-r / 2} h\right\rangle d^{*} r=\left\langle b\left(g^{\prime}\right), f_{0}\right\rangle .
$$

4.2. Интегральная модель представления группы токов $P^{X}$. Перейдем к основной конструкции этого раздела. Свяжем с каждым каноническим представлением $T$ подгруппы $P_{0}$ в гильбертовом пространстве $H$ с почти инвариантным относительно $T$ вектором $h \in H$ представление $U$ группы $P^{X}$, которое назовем интегральной моделью, ассоциированной с представлением $T$ подгруппы $P_{0}$, и обозначим через INT $T$.

Конструкция представления INT $T$ группы $P^{X}$ параллельна приведенной ранее конструкции представления $\widetilde{T}$ группы $P$ по представлению $T$ подгруппы $P_{0}$. Именно, мы заменяем в этой конструкции пространства $H_{r}, r \in \mathbb{R}_{+}^{*}$, с действующими на них представлениями $T_{r}$ группы $P_{0}$ пространствами $H_{\xi}$, $\xi=\left\{r_{k}, x_{k}\right\} \in l_{+}^{1}(X)$, с действующими на них представлениями $T_{\xi}$ группы $P_{0}^{X}$, 
а прямой интеграл по мере $r^{-1} d r$ на $\mathbb{R}_{+}^{*}$ пространств $H_{r}$ - прямым интегралом по мере $d \mathscr{L}(\xi)$ на конусе $l_{+}^{1}(X)$ пространств $H_{\xi}$. Таким образом, согласно этой конструкции, интегральная модель представления группы $P^{X}$, ассоциированная с каноническим представлением $T$ группы $P_{0}$, реализуется в прямом интеграле по мере $\mathscr{L}$ гильбертовых пространств $H_{\xi}$,

$$
\operatorname{INT} H=\int_{l_{+}^{1}(X)} H_{\xi} d \mathscr{L}(\xi), \quad H_{\xi}=\bigotimes_{k=1}^{\infty} H_{r_{k}},
$$

т. е. в пространстве сечений $F(\xi)=F\left(\left\{r_{k}, x_{k}\right\}\right)$ расслоения над конусом $l_{+}^{1}(X)$, где слоем над каждой точкой $\xi=\left\{r_{k}, x_{k}\right\}$ является счетное тензорное произведение $H_{\xi}=\bigotimes_{k=1}^{\infty} H_{r_{k}}, H_{r_{k}}=H$. (От $\xi$ этот слой как гильбертово пространство не зависит, но само представление зависит.) Действие группы $P_{0}^{X}$ в каждом слое этого расслоения индуцирует представление этой группы на всем пространстве INT $H$ :

$$
U(g)\left(\bigotimes_{k=1}^{\infty} f_{k}\left(r_{k}\right)\right)=\bigotimes_{k=1}^{\infty} T\left(g\left(x_{k}\right)\right) f_{k}\left(r_{k}\right) \quad \text { при } g \in P_{0}^{X} .
$$

Определим операторы $U\left(r_{0}(\cdot)\right)$ представления группы $\mathbb{R}_{+}^{X}$ в пространстве INT $H$ следующей формулой:

$$
\left(U\left(r_{0}(\cdot)\right) F\right)\left(\left\{r_{k}, x_{k}\right\}\right)=\exp \left(\frac{1}{2} \int_{X} \log r_{0}(x) d m(x)\right) F\left(\left\{r_{0}\left(x_{k}\right) r_{k}, x_{k}\right\}\right)
$$

для любого $r(\cdot) \in \mathbb{R}_{+}^{X}$. Таким образом, эти операторы переставляют слои расслоения INT $H$.

ПреДЛОЖенИЕ 4.3. Операторъ $U\left(r_{0}(\cdot)\right), r_{0}(\cdot) \in \mathbb{R}_{+}^{X}$, ортогоналъны (унитарны) и порождают вместе с операторами подгруппы $P_{0}^{X}$ ортогональное (унитарное) представление группы токов $P^{X}$, инвариантное относительно преобразованиц $X$, сохраняющих меру $m$ на $X$ :

$$
U(g)\left(\bigotimes_{k=1}^{\infty} f_{k}\left(r_{k}\right)\right)=\exp \left(\frac{1}{2} \int_{X} \log r_{0}(x) d m(x)\right) \bigotimes_{k=1}^{\infty} \widetilde{T}\left(g\left(x_{k}\right) f_{k}\right)\left(r_{k}\right)
$$

для любого $g=r_{0} g_{0} \in P^{X}\left(r_{0} \in\left(\mathbb{R}_{+}^{*}\right)^{X}, g_{0} \in P_{0}^{X}\right)$, где $\widetilde{T}-$ представление группы $P$, ассоииированное с представлением $T$ подгруппы $P_{0}$.

ДокАЗАТЕЛЬство. Ортогональность (унитарность) операторов $U\left(r_{0}(\cdot)\right)$ следует из проективной инвариантности меры $\mathscr{L}$, см. (2.3). В самом деле,

$$
\begin{aligned}
\left\|U\left(r_{0}(\cdot)\right) F\right\|^{2} & =\exp \left(\int_{X} \log r_{0}(x) d m(x)\right) \int_{l_{+}^{1}}\left\|F\left(r_{0}(\cdot) \xi\right)\right\|^{2} d \mathscr{L}(\xi) \\
& =\exp \left(\int_{X} \log r_{0}(x) d m(x)\right) \int_{l_{+}^{1}}\|F(\xi)\|^{2} d \mathscr{L}\left(r_{0}^{-1}(\cdot) \xi\right)=\|F\|^{2},
\end{aligned}
$$

поскольку $d \mathscr{L}\left(r_{0}^{-1}(\cdot) \xi\right)=\exp \left(-\int_{X} \log r_{0}(x) d m(x)\right) d \mathscr{L}(\xi)$. 
Далее, из формул для операторов следует, что выполняется соотношение:

$$
U^{-1}\left(r_{0}(\cdot)\right) U\left(g_{0}(\cdot)\right) U\left(r_{0}(\cdot)\right)=U\left(r_{0}^{-1}(\cdot) g_{0}(\cdot) r_{0}(\cdot)\right)
$$

для любых $g_{0}(\cdot) \in P_{0}^{X}$ и $r_{0}(\cdot) \in \mathbb{R}_{+}^{X}$. Значит, эти операторы порождают представление группы $P^{X}$.

Поскольку мера $\mathscr{L}$ на $l_{+}^{1}(X)$ сохраняется при любых преобразованиях $X$, сохраняющих меру $m$ на $X$, то полученное представление группы $P^{X}$ также инвариантно относительно этих преобразований. Предложение 4.3 доказано.

На основании (4.5) выражение (4.9) для $U(g), g \in P^{X}$, может быть представлено в виде

$$
U(g)\left(\bigotimes_{k=1}^{\infty} f_{k}\left(r_{k}\right)\right)=\exp \left(-\int_{X} \lambda(g(x)) d m(x)\right) \bigotimes_{k=1}^{\infty} \widetilde{T}\left(g\left(x_{k}\right) f_{k}\right)\left(r_{k}\right)
$$

где

$$
\lambda(g)=\frac{1}{2}\|b(g)\|^{2}+\operatorname{Re} c(g) .
$$

Мы называем построенное представление группы $P^{X}$ интегральной моделью, ассоциированной с каноническим представлением $T$ группы $P_{0}$, и обозначаем его, по аналогии фоковскими представлениями, через INT $T$, а через INT $H$ обозначаем гильбертово пространство, в котором оно реализовано.

Из описания интегральной модели вытекает следующее утверждение.

Теорема 4.2. Если $T_{1}$ и $T_{2}$ - канонические представления группь $P_{0}$ в пространствах $\mathrm{H}_{1}$ и $\mathrm{H}_{2}$, mo

$$
\operatorname{INT}\left(H_{1} \oplus H_{2}\right)=\operatorname{INT} H_{1} \otimes \operatorname{INT} H_{2},
$$

и в этом пространстве действует интегральная модель $\operatorname{INT}\left(T_{1} \oplus T_{2}\right)$ представления группы $G^{X}$, ассочиированная с представлением $T_{1} \oplus T_{2}$ группы $P_{0}$.

Теорема 4.3. Если каноническое представление $T$ группы $P_{0}$ неприводимо, то ассоциированное с $T$ представление $U=\operatorname{INT} T$ группы токов $P^{X}$ такоее неприводимо.

Доказательство. Рассмотрим сначала операторы $U(g(\cdot)), g(\cdot) \in P_{0}^{X}$. Они сохраняют слои $H_{\xi}$ расслоения INT $H$, и, в силу предложения 3.3 , определенные ими на этих слоях представления группы $P_{0}^{X}$ неприводимы и попарно не эквивалентны. Поэтому любой оператор $A$ на INT $H$, перестановочный с этими операторами, кратен единичному оператору на каждом слое расслоения INT $H$, т. е. является оператором умножения на функцию $a(\xi)=a\left(\left\{r_{k}, x_{k}\right\}\right)$. Если этот оператор $A$ перестановочен также с операторами $U\left(r_{0}(\cdot)\right), r_{0}(\cdot) \in \mathbb{R}_{+}^{X}$, то функция $a\left(\left\{r_{k}, x_{k}\right\}\right)$ постоянна на орбитах группы мультипликаторов $\mathscr{M}$, действующей в $l_{+}^{1}(X)$ умножением на положительные функции $r_{0}(\cdot):\left\{r_{k}, x_{k}\right\} \mapsto$ $\left\{r_{0}\left(x_{k}\right) r_{k}, x_{k}\right\}$. Отсюда, в силу эргодичности меры $\mathscr{L}$, следует, что оператор $A$ есть константа. 
4.3. Тотальное подмножество $M \subset$ INT $H$. Обозначим через $\Omega$ и назовем вакуумным вектор в пространстве INT $H$ представления группы $P^{X}$ вида:

$$
\Omega(\xi)=\bigotimes_{k=1}^{\infty} f_{0}\left(r_{k}\right) \quad \text { при } \xi=\left\{r_{k}, x_{k}\right\}, \quad \text { где } f_{0}(r)=e^{-r / 2} h_{r} .
$$

Из формулы для характеристического функционала меры $\mathscr{L}$ следует, что $\|\Omega\|=1$.

ОПРЕДЕЛЕНИЕ 4. Поставим в соответствие каждому элементу $g \in P^{X}$ следующий вектор в пространстве INT $H$ :

$$
F_{g}(\xi)=\exp \left(\int_{X}\left(\frac{1}{2}\|b(g(x))\|^{2}-i \operatorname{Im} c(g(x))\right) d m(x)\right) U(g) \Omega(\xi),
$$

где $c(g)=\left\langle b(g), f_{0}\right\rangle$.

В силу цикличности $\Omega$, множество $M$ векторов $F_{g}, g \in P^{X}$, тотально в пространстве представления.

Поскольку

$$
(U(g) \Omega)(\xi)=\exp \left(-\int_{X} \lambda(g(x)) d m(x)\right) \bigotimes_{k=1}^{\infty}\left(\widetilde{T}\left(g\left(x_{k}\right)\right) f_{0}\right)\left(r_{k}\right) \quad \text { при } \xi=\left\{r_{k}, x_{k}\right\},
$$

где $\lambda(g)=\frac{1}{2}\|b(g)\|^{2}+\operatorname{Re} c(g)$, выражение для $F_{g}$ можно представить в виде

$$
F_{g}(\xi)=\exp \left(-\int_{X} c(g(x)) d m(x)\right) \bigotimes_{k=1}^{\infty}\left(\widetilde{T}\left(g\left(x_{k}\right)\right) f_{0}\right)\left(r_{k}\right) \quad \text { при } \xi=\left\{r_{k}, x_{k}\right\} .
$$

Вектор $\Omega=F_{e}$ и порожденное им множество векторов $F_{g}$ можно рассматривать как аналоги вакуумного вектора $\operatorname{EXP} 0$ и порожденного им множества векторов $\operatorname{EXP} b^{X}(g)$ в пространстве фоковского представления. Приведем основные свойства множества $M$.

Из определения векторов $F_{g}$ вытекает следующее утверждение.

ПреДЛОЖеНИЕ 4.4. Операторы представления действуют на векторы $F_{g}$ по формуле

$$
U(g) F_{g_{1}}=\exp \left(-\int_{X}\left(c\left(g_{1}(x)\right)-c\left(g g_{1}(x)\right)+\lambda(g(x))\right) d m(x)\right) F_{g g_{1}},
$$

где $\lambda(g)=\frac{1}{2}\|b(g)\|^{2}+\operatorname{Re} c(g)$.

В самом деле, имеем:

$$
\begin{aligned}
\left(U(g) F_{g_{1}}\right)(\xi) & =\exp \left(-\int_{X} c\left(g_{1}(x)\right) d m(x)\right) U(g)\left(\bigotimes_{k=1}^{\infty} \widetilde{T}\left(g_{1}\left(x_{k}\right) f_{0}\right)\left(r_{k}\right)\right) \\
& =\exp \left(-\int_{X}\left(c\left(g_{1}(x)\right)+\lambda(g(x))\right) d m(x)\right)\left(\bigotimes_{k=1}^{\infty} \widetilde{T}\left(g g_{1}\left(x_{k}\right) f_{0}\right)\left(r_{k}\right)\right) \\
& =\exp \left(-\int_{X}\left(c\left(g_{1}(x)\right)+\lambda(g(x))-c\left(g g_{1}(x)\right)\right) d m(x)\right) F_{g g_{1} .}
\end{aligned}
$$


ПрЕДЛОжЕНИЕ 4.5. Скалярное произведение на множестве векторов $F_{g}$ задается формулой

$$
\left\langle F_{g_{1}}, F_{g_{2}}\right\rangle=\exp \left(\int_{X}\left\langle b\left(g_{1}(x)\right), b\left(g_{2}(x)\right)\right\rangle d m(x)\right) \quad \text { для всех } g_{1}, g_{2} \in P^{X}
$$

ДокАЗАТЕльство. Из определения векторов $F_{g}$ следует:

$$
\left\langle F_{g_{1}}, F_{g_{2}}\right\rangle=\exp \left(-\int_{X}\left(c\left(g_{1}(x)\right)+\overline{c\left(g_{2}(x)\right)}\right) d m(x)\right) I,
$$

где

$$
I=\int_{l_{+}^{1}(X)} \prod_{k=1}^{\infty}\left\langle\left(\widetilde{T}\left(g_{1}\left(x_{k}\right)\right) f_{0}\right)\left(r_{k}\right),\left(\widetilde{T}\left(g_{2}\left(x_{k}\right)\right) f_{0}\right)\left(r_{k}\right)\right\rangle_{H} d \mathscr{L}(\xi) .
$$

Для вычисления $I$ воспользуемся общей формулой (2.6). Обозначим

$$
\varphi(r, x)=\left\langle\left(\widetilde{T}\left(g_{1}(x)\right) f_{0}\right)(r),\left(\widetilde{T}\left(g_{2}(x)\right) f_{0}\right)(r)\right\rangle_{H} .
$$

Отсюда на основании (2.6) получаем:

$$
I=\exp \left(\int_{X} \int_{0}^{\infty}\left(\varphi(r, x)-e^{-r}\right) d^{*} r d m(x)\right) .
$$

Преобразуем подынтегральное выражение. Поскольку $\widetilde{T}\left(g_{1}(x)\right) f_{0}=b(g(x))+f_{0}$ и $e^{-r}=\left\langle f_{0}(r), f_{0}(r)\right\rangle_{H}$, функцию $\varphi(r, x)$ можно представить в виде

$$
\varphi(r, x)=\left.\left\langle b\left(g_{1}(x), r\right), b\left(g_{2}(x), r\right)\right\rangle\right|_{H}+\left.\left\langle b\left(g_{1}(x), r\right), f_{0}(r)\right\rangle\right|_{H}+\overline{\left\langle b\left(g_{2}(x), r\right), f_{0}(r)\right\rangle_{H}} .
$$

Интегрируя это выражение по $r$, получаем:

$$
I=\exp \left(\int_{X}\left(\left\langle b\left(g_{1}(x)\right), b\left(g_{2}(x)\right)\right\rangle+c\left(g_{1}(x)\right)+\overline{c\left(g_{2}(x)\right)}\right) d m(x)\right) .
$$

Отсюда и из (4.18) получаем (4.17).

ЗАмечАниЕ. Обозначим через $K$ подгруппу элементов $k \in P$ таких, что $b(k)=0$. Очевидно, что два вектора $F_{g_{1}}$ и $F_{g_{2}}$ совпадают тогда и только тогда, когда $g_{2}=g_{1} k$, где $k \in K^{X}$. Поэтому множество попарно различных векторов $F_{g}$ естественно отождествляется с факторпространством $G^{X} / K^{X}$.

\section{4. Сферическая функция представления $U=\operatorname{INT} T$.}

ОПРЕДЕЛЕНИЕ 5. Сферической функцией представления $U=\operatorname{INT} T$ группы $P^{X}$ назовем следующую функцию на $P^{X}$ :

$$
\Psi(g)=\langle U(g) \Omega, \Omega\rangle, \quad \text { где } \Omega(\xi)=\bigotimes_{k=1}^{\infty} f_{0}\left(r_{k}\right) \text { при } \xi=\left\{r_{k}, x_{k}\right\} .
$$

Поскольку представление $U$ неприводимо, эта функция определяет его однозначно, с точностью до эквивалентности. 
Tеорема 4.4. Сферическая функиия $\Psi(g)$ представима в виде:

$$
\Psi(g)=\exp \left(\int_{X}\left(i \operatorname{Im} c(g(x))-\frac{1}{2}\|b(g(x))\|^{2}\right) d m(x)\right),
$$

где $b(g)$ - 1-кочикл представления $\widetilde{T}$ группы $P$, ассочиированного с представлением $T$ подгруппы $P_{0}$ :

$$
b(g)=\left(\widetilde{T}(g) f_{0}\right)(r)-f_{0}(r), \quad f_{0}(r)=e^{-r / 2}
$$

$u c(g)=\left\langle b(g(x)), f_{0}\right\rangle$.

ДокАЗАТЕЛЬство. Формула (4.21) следует из формулы (4.17) для скалярного произведения векторов $F_{g}$ и формулы $(4.13)$, выражающей $F_{g}$ через вектор $U(g) \Omega$. Для полноты приведем ее прямой вывод. Имеем на основании (4.14):

$$
\Psi(g)=\exp \left(-\int_{X} \lambda(g(x)) d m(x)\right) \int_{l_{+}^{1}(X)}\left(\prod_{k=1}^{\infty}\left\langle\widetilde{T}\left(g\left(x_{k}\right) f_{0}\right)\left(r_{k}\right), f_{0}\left(r_{k}\right)\right\rangle\right) d \mathscr{L}(\xi),
$$

где $\lambda(g)=\frac{1}{2}\|b(g)\|^{2}+\operatorname{Re} c(g)$. Для вычисления интеграла

$$
I=\int_{l_{+}^{1}(X)}\left(\prod_{k=1}^{\infty}\left\langle\widetilde{T}\left(g\left(x_{k}\right) f_{0}\right)\left(r_{k}\right), f_{0}\left(r_{k}\right)\right\rangle\right) d \mathscr{L}(\xi)
$$

перейдем к проекциям конуса $l_{+}^{1}(X)$ на конечномерные конусы $\Phi_{\alpha}$, ассоциированные с разбиениями $\alpha: X=\bigcup_{k=1}^{m} X_{k}$ пространства $X$. В проекции на $\Phi_{\alpha}$ выражение для $I$ принимает вид:

$$
I_{\alpha}=\prod_{k=1}^{m} I_{\alpha}^{k}, \quad \text { где } I_{\alpha}^{k}=\int_{0}^{\infty}\left\langle\left(\widetilde{T}\left(g_{k}\right) f_{0}\right)\left(r_{k}\right), f_{0}\left(r_{k}\right)\right\rangle_{H} \frac{r_{k}^{\lambda_{k}-1} d r_{k}}{\Gamma\left(\lambda_{k}\right)} .
$$

Здесь обозначено: $g_{k}=\left.g(x)\right|_{X_{k}}$. Подставим сюда выражение для $\widetilde{T}\left(g_{k}\right) f_{0}$ через нетривиальный 1-коцикл $b(g, r)$ :

$$
\left(\widetilde{T}\left(g_{k}\right) f_{0}\right)\left(r_{k}\right)=b\left(g_{k}, r_{k}\right)+f_{0}\left(r_{k}\right) .
$$

Учитывая, что $\left.\int_{0}^{\infty}\left\|f_{0}(r)\right\|^{2}\right|_{H} \frac{r^{\lambda_{k}-1} d r_{k}}{\Gamma\left(\lambda_{k}\right)}=1$, получаем

$$
I_{\alpha}^{k}=1+\int_{0}^{\infty}\left\langle b\left(g_{k}, r\right), f_{0}(r)\right\rangle_{H} \frac{r^{\lambda_{k}-1} d r}{\Gamma\left(\lambda_{k}\right)} .
$$

Отсюда следует, что

$$
I_{\alpha}^{k}=1+\lambda_{k} \int_{0}^{\infty}\left\langle b\left(g_{k}, r\right), f_{0}(r)\right\rangle r^{-1} d r+O\left(\lambda_{k}^{2}\right)=1+\lambda_{k} c\left(g_{k}\right)+O\left(\lambda_{k}^{2}\right) .
$$

Следовательно, $I_{\alpha}=\prod_{k=1}^{m}\left(1+c\left(g_{k}\right)+O\left(\lambda_{k}^{2}\right)\right)$. Так как

$$
\sum \lambda_{k} c\left(g_{k}\right)=\int_{X} c(g(x)) d m(x)
$$


где $g(x)$ - кусочно постоянная функция со значениями $g_{k}$ на элементах разбиения $\alpha$, полученное выражение, с точностью до членов порядка выше 1 относительно $\lambda_{k}$, можно представить в виде: $I_{\alpha} \sim \exp \left(\int_{X} c(g(x)) d m(x)\right)$. Переходя здесь к индуктивному пределу по множеству конечных разбиений $\alpha$, получаем выражение для $I$ :

$$
I=\exp \left(\int_{X} c(g(x)) d m(x)\right) .
$$

Отсюда и из (4.23) следует формула (4.21) для $\Psi(g)$.

4.5. Связь между интегральной и фоковской моделями представлений группы токов $P^{X}=\left(\mathbb{R}_{+}^{*} \curlywedge P_{0}\right)^{X}$. Фоковская конструкция (см., например, [1], [3], [30], [28], [31]) относит каждой паре $(\widetilde{T}, b)$, где $\widetilde{T}$ - особое ортогональное или унитарное представление произвольной локально компактной группы $G$ в гильбертовом пространстве $\mathscr{H}$, а $b$ - нетривиальный 1-коцикл $b: G \rightarrow \mathscr{H}$, унитарное представление группы токов $G^{X}$ в комплексном гильбертовом пространстве ЕХР $\mathscr{H}^{X}$, где

$$
\mathscr{H}^{X}=\int_{X}^{\oplus} \mathscr{H}_{x} d m(x), \quad \mathscr{H}_{x}=\mathscr{H} .
$$

По определению,

$$
\operatorname{EXP} \mathscr{H}^{X}=\bigoplus_{k=0}^{\infty} S^{k} \mathscr{H}^{X}
$$

$\left(S^{k}-k\right.$-я симметризованная тензорная степень) в случае, когда $\mathscr{H}$ - комплексное гильбертово пространство, а в случае вещественного пространства $\mathscr{H}$ пространство ЕХР $\mathscr{H}^{X}$ является окомплексиванием вещественного пространства $\bigoplus_{k=0}^{\infty} S^{k} \mathscr{H}^{X}$. В последнем случае оно изоморфно фоковскому пространству $\operatorname{EXP}\left(\mathscr{H}_{\mathbb{C}}\right)^{X}$, где $\mathscr{H}_{\mathbb{C}}$ - окомплексивание вещественного пространства $\mathscr{H}$.

В пространстве ЕХР $\mathscr{H}^{X}$ вводится тотальное подмножество векторов $\mathrm{EXP} v$, $v \in \mathscr{H}^{X}$, вида

$$
\operatorname{EXP} v=\mathbb{I} \oplus v \oplus \frac{1}{\sqrt{2 !}} v \otimes v \oplus \frac{1}{\sqrt{3 !}} v \otimes v \otimes v \oplus \cdots
$$

и операторы представления задаются на этом подмножестве векторов следующей формулой:

$$
U(g) \operatorname{EXP} v=\exp \left(-\frac{1}{2}\left\|b^{X}(g)\right\|^{2}-\left\langle\widetilde{T}^{X}(g) v, b^{X}(g)\right\rangle\right) \operatorname{EXP}\left(\widetilde{T}^{X}(g) v+b^{X}(g)\right) .
$$

Здесь через $\widetilde{T}^{X}$ и $b^{X}$ обозначены соответственно представление группы $G^{X}$ в пространстве $\mathscr{H}^{X}$, порожденное представлением $\widetilde{T}$ группы $G$ в пространстве $\mathscr{H}$, и 1 -коцикл $G^{X} \rightarrow \mathscr{H}^{X}$, порожденный 1-коциклом $b: G \rightarrow \mathscr{H}$.

Операторы $U(g)$ связаны соотношением

$$
U\left(g_{1} g_{2}\right)=\exp \left(i \operatorname{Im}\left\langle T^{X}\left(g_{1}\right) b^{X}\left(g_{2}\right), b^{X}\left(g_{1}\right)\right\rangle\right) U\left(g_{1}\right) U\left(g_{2}\right) \quad \text { для любых } g_{1}, g_{2} \in G^{X} .
$$


Таким образом, фоковские представления группы $G^{X}$, связанные с унитарными представлениями группы $G$, проективны, если 2-коцикл

$$
\exp \left(i \operatorname{Im}\left\langle T^{X}\left(g_{1}\right) b^{X}\left(g_{2}\right), b^{X}\left(g_{1}\right)\right\rangle\right)
$$

не равен тождественно нулю, и эквивалентны настоящим тогда и только тогда, когда этот 2-коцикл тривиален.

ТЕорема 4.5. Фоковская модель представления группь $P^{X}=\left(\mathbb{R}_{+}^{*}<P_{0}\right)^{X}$ в пространстве ЕХP $\mathscr{H}^{X}$ проективно эквивалентна ее настоящему представлению $V$ в том же пространстве ЕХР $\mathscr{H}^{X}$, операторы которого связаны с операторами $U$ фоковского представления соотношением

$$
V(g)=\exp \left(i \operatorname{Im} \int_{X}\left\langle b(g(x)), f_{0}\right\rangle d m(x)\right) U(g) .
$$

В самом деле, из (4.7) следует, что 2-коцикл

$$
\lambda\left(g_{1}, g_{2}\right)=\exp \left(i \operatorname{Im}\left\langle T^{X}\left(g_{1}\right) b^{X}\left(g_{2}\right), b^{X}\left(g_{1}\right)\right\rangle\right)
$$

в соотношении (4.24) тривиален, а именно,

$$
\lambda\left(g_{1}, g_{2}\right)=\frac{C\left(g_{1}\right) C\left(g_{2}\right)}{C\left(g_{1} g_{2}\right)}, \quad \text { где } C(g)=\exp \left(i \operatorname{Im} \int_{X}\left\langle b(g(x)), f_{0}\right\rangle d m(x)\right) .
$$

Отсюда следует утверждение.

Определим сферическую функцию представления $V$ группы $P^{X}$ в пространстве EXP $\mathscr{H}^{X}$ равенством

$$
\Phi(g)=\langle V(g) \operatorname{EXP} 0, \operatorname{EXP} 0\rangle .
$$

Из определения фоковского представления $U$ и проективно эквивалентного ему (настоящего) представления $V$ группы $P^{X}$ вытекает следующее утверждение.

ПреДЛОЖЕНИЕ 4.6. Сферическая функиия $\Phi(g)$ представления $V$ группьи $P^{X}$ имеет вид:

$$
\Phi(g)=\exp \left(\int_{X}\left(i \operatorname{Im}\left\langle b(g(x)), f_{0}\right\rangle-\frac{1}{2}\|b(g(x))\|^{2}\right) d m(x)\right) .
$$

ТеОРема 4.6. Пусть $T$ - каноническое неприводимое представление группь $P_{0}$ в пространстве $H, \widetilde{T}$ - ассочиированное с ним представление группы $P=\mathbb{R}_{+}^{*} \curlywedge P_{0}$ в пространстве $\mathscr{H}$ и $b(g, r)=\left(\widetilde{T}(g) f_{0}\right)(r)-f_{0}(r)$, где $f_{0}(r)=$ $e^{-r / 2} h,-$ нетривиальный 1-коцикл $P \rightarrow \mathscr{H}$. Тогда интегральная модель INT T представления группы $P^{X}$ в пространстве INT $H$ проективно эквивалентна ее фоковскому представлению $U$ в пространстве ЕXP $\mathscr{H}^{X}$. Сплетающий оператор этих представлений порожден отображением ииклических векторов $\Omega \rightarrow \operatorname{EXP} 0$.

ДокАЗАТЕЛЬСТВО. Заметим, что формулы (4.21) и (4.26) для сферической функции представления INT $T$ группы $P^{X}$ и сферической функции ее представления $V$ в фоковском пространстве совпадают. Следовательно, эти представления эквивалентны, и их сплетающий оператор порожден отображением циклических векторов $\Omega \rightarrow \operatorname{EXP~0.~Отсюда~и~из~теоремы~} 4.5$ следует утверждение. 
4.6. Продолжение интегральной модели представления группы $P^{X}$ до представления группы $G^{X}$, где $P \subset G$. Рассмотрим произвольную локально компактную группу $G$, содержащую группу $P=\mathbb{R}_{+}^{*} \wedge P_{0}$ в качестве своей подгруппы. Пусть $T$ - неприводимое каноническое представление подгруппы $P_{0}$ в пространстве $H, \widetilde{T}$ - ассоциированное с $T$ особое представление группы $P$ в пространстве $\mathscr{H}=\int_{0}^{\infty} H_{r} d^{*} r, H_{r}=H$ и $b(g): P \rightarrow \mathscr{H}$ - нетривиальный 1-коцикл представления $\widetilde{T}$, заданный равенством:

$$
b(g)=\widetilde{T}(g) f_{0}-f_{0}, \quad \text { где } f_{0}(r)=e^{-r / 2} h_{r} .
$$

ТЕОРема 4.7. Предположим, что существует продолжение представления $\widetilde{T}$ группы $P$ в пространстве $\mathscr{H}$ до представления группы $G$ и определено продолжение 1-коцикла (4.27) группь $P$ до 1-коцикла того же вида группь $G$. Тогда существует ассощиированное с этим продолжением продолжение интегральной модели INT $T$ представления $U$ группы токов $P^{X}$ до представления группь токов $G^{X}$.

Опишем явно это продолжение. Ранее в п. 4.3 в пространстве INT $H$ представления группы $P^{X}$ было введено тотальное множество векторов вида

$$
F_{g}(\xi)=\exp \left(-\int_{X} c(g(x)) d m(x)\right) \bigotimes_{k=1}^{\infty}\left(\widetilde{T}\left(g\left(x_{k}\right)\right) f_{0}\right)\left(r_{k}\right) \quad \text { при } \xi=\left\{r_{k}, x_{k}\right\}
$$

где $f_{0}(r)=\exp (-r / 2) h_{r}, c(g)=\left\langle b(g), f_{0}\right\rangle$.

Было доказано, что

$$
\left\langle F_{g_{1}}, F_{g_{2}}\right\rangle=\exp \left(\int_{X} c\left(g_{1}(x), g_{2}(x)\right) d m(x)\right)
$$

где

$$
c\left(g_{1}, g_{2}\right)=\left\langle b\left(g_{1}\right), b\left(g_{2}\right)\right\rangle,
$$

а операторы представления группы $P^{X}$ действуют на эти векторы по формуле

$$
U(g) F_{g_{1}}=\exp \left(-\int_{X}\left(c\left(g_{1}(x)\right)-c\left(g_{1}(x)\right)+\lambda(g(x))\right) d m(x)\right) F_{g g_{1}},
$$

где $\lambda(g)=\frac{1}{2}\|b(g)\|^{2}+\operatorname{Re} c(g)$.

ОПРЕДЕлЕниЕ 6. Расширим семейство функций $F_{g}, g \in P^{X}$, определив $F_{g}$ при произвольном $g \in G^{X}$ той же формулой (4.28).

Дословно так же, как и в предложении 4.5, устанавливается: скалярное произведение на множестве векторов $F_{g}, g \in G^{X}$, задается той же формулой (4.29). В частности, $\left\langle F_{g}, F_{g}\right\rangle=\exp \left(\int_{X} c(g(x), g(x)) d m(x)\right)<\infty$, а потому векторы $F_{g}, g \in G^{X}$, принадлежат пространству INT $H$. 
ОПредЕЛЕНиЕ 7. Зададим на множестве векторов $F_{g}, g \in G^{X}$, действие операторов $U(g)$ группы $G^{X}$ следующей формулой:

$$
U(g) F_{g_{1}}=\exp \left(-\int_{X} \lambda\left(g(x), g_{1}(x)\right) d m(x)\right) F_{g g_{1}},
$$

где

$$
\lambda\left(g, g_{1}\right)=\frac{1}{2}\|b(g)\|^{2}+\left\langle\widetilde{T}(g) b\left(g_{1}\right), b(g)\right\rangle-i \operatorname{Im} c(g) .
$$

ТеОрема 4.8. Oператоры $U(g)$ группъ $G^{X}$ сохраняют скалярнъе произведения векторов $F_{g}$, m.e.

$$
\left\langle U(g) F_{g_{1}}, U(g) F_{g_{2}}\right\rangle=\left\langle F_{g_{1}}, F_{g_{2}}\right\rangle \quad \text { для любих } g, g_{1}, g_{2} \in G^{X},
$$

а потому продолжаются до ортогоналъных (унитарных) операторов на всем пространстве INT $H$.

ДоКАЗАТЕЛЬСТВо. Имеем:

$$
\left\langle U(g) F_{g_{1}}, U(g) F_{g_{2}}\right\rangle=\exp \left(-\int_{X} u\left(g(x), g_{1}(x), g_{2}(x)\right) d m(x)\right),
$$

где

$$
u\left(g, g_{1}, g_{2}\right)=\lambda\left(g, g_{1}\right)+\overline{\lambda\left(g, g_{2}\right)}-\left\langle b\left(g g_{1}\right), b\left(g g_{2}\right)\right\rangle .
$$

Так как $b\left(g g_{1}\right)=\widetilde{T}(g) b\left(g_{1}\right)+b(g)$, то отсюда и из выражения (4.33) для $\lambda\left(g, g_{i}\right)$ следует, что $u\left(g, g_{1}, g_{2}\right)=\left\langle b\left(g_{1}\right), b\left(g_{2}\right)\right\rangle$. Отсюда следует (4.34).

ТЕорема 4.9. Операторы $U(g)$ задают, вообще говоря, проективное представление группъ $G^{X}$ в пространстве INT $H$ :

$$
U\left(g_{1} g_{2}\right)=\exp \left(i \operatorname{Im} \int_{X} p\left(g_{1}(x), g_{2}(x)\right) d m(x)\right) U\left(g_{1}\right) U\left(g_{2}\right) \text { для любъх } g_{1}, g_{2} \in G^{X},
$$

əde

$$
p\left(g_{1}, g_{2}\right)=\left\langle\widetilde{T}\left(g_{1}\right) b\left(g_{2}\right), b\left(g_{1}\right)\right\rangle-c\left(g_{1}\right)-c\left(g_{2}\right)+c\left(g_{1} g_{2}\right) .
$$

ДокАЗАТЕльство. Имеем для любых $g, g_{1}, g_{2} \in G^{X}$ :

$$
\begin{aligned}
U\left(g_{1}\right) U\left(g_{2}\right) F_{g} & =\exp \left(-\int_{X} a\left(g_{1}(x), g_{2}(x), g(x)\right) d m(x)\right) F_{g_{1} g_{2} g}, \\
U\left(g_{1} g_{2}\right) F_{g} & =\exp \left(-\int_{X} a^{\prime}\left(g_{1}(x), g_{2}(x), g(x)\right) d m(x)\right) F_{g_{1} g_{2} g},
\end{aligned}
$$

где

$$
a\left(g_{1}, g_{2}, g\right)=\lambda\left(g_{2}, g\right)+\lambda\left(g_{1}, g_{2} g\right), \quad a^{\prime}\left(g_{1}, g_{2}, g\right)=\lambda\left(g_{1} g_{2}, g\right) .
$$

Воспользуемся соотношением

$$
l\left(g_{2}, g\right)+l\left(g_{1}, g_{2} g\right)-l\left(g_{1} g_{2}, g\right)=i \operatorname{Im}\left\langle\widetilde{T}\left(g_{1}\right) b\left(g_{2}\right), b\left(g_{1}\right)\right\rangle
$$

при

$$
l\left(g_{1}, g_{2}\right)=\frac{1}{2}\left\|b\left(g_{1}\right)\right\|^{2}+\left\langle\widetilde{T}\left(g_{1}\right) b\left(g_{2}\right), b\left(g_{1}\right)\right\rangle .
$$


Из него следует, что

$$
a\left(g_{1}, g_{2}, g\right)-a^{\prime}\left(g_{1}, g_{2}, g\right)=i \operatorname{Im}\left(\left\langle\widetilde{T}\left(g_{1}\right) b\left(g_{2}\right), b\left(g_{1}\right)\right\rangle-c\left(g_{1}\right)-c\left(g_{2}\right)+c\left(g_{1} g_{2}\right)\right) .
$$

Значит,

$$
\begin{aligned}
& U\left(g_{1}\right) U\left(g_{2}\right) U^{-1}\left(g_{1} g_{2}\right) F_{g} \\
& =\exp \left(-\operatorname{Im} \int_{X} p\left(g_{1}(x), g_{2}(x)\right) d m(x)\right) F_{g} \quad \text { при любом } g \in G^{X},
\end{aligned}
$$

где $p\left(g_{1}, g_{2}\right)$ задается равенством (4.36). Отсюда следует утверждение.

Теорема 4.10. Ограничение представления $U$ группы $G^{X}$ на подгруппу $P^{X}$ совпадает с исходным представлением INT $T$.

ДокАЗАтЕльство. Достаточно убедиться, что на тотальном подмножестве векторов $F_{g_{1}}$ операторы $U(g)$ совпадают при $g \in P^{X}$ с исходными операторами. При $g, g_{1} \in P$ имеем согласно (4.7): $\left\langle\widetilde{T}(g) b\left(g_{1}\right), b(g)\right\rangle=-c\left(g g_{1}\right)+c(g)+c\left(g_{1}\right)$. Значит,

$$
\lambda\left(g, g_{1}\right)=c\left(g_{1}\right)-c\left(g g_{1}\right)+\frac{1}{2}\|b(g)\|^{2}+\operatorname{Re} c(g),
$$

а потому выражение для $U(g) F_{g_{1}}$ совпадает при $g, g_{1} \in P^{X}$ с исходным выражением (4.16).

\section{5. Интегральная модель представления группы токов $\mathrm{O}(n, 1)^{X}, n>2$}

Этот и последующие разделы посвящены описанию интегральных моделей представлений групп токов $P^{X}$, где $P$ - максимальная параболическая подгруппа группы $\mathrm{O}(n, 1), \mathrm{U}(n, 1)$ или $\mathrm{Sp}(n, 1)$, и в двух первых случаях продолжению этих представлений групп $P^{X}$ до представлений соответственно групп $\mathrm{O}(n, 1)^{X}$ и $\mathrm{U}(n, 1)^{X}$. (Для группы $\mathrm{Sp}(n, 1)$ представления ее группы токов не существует.) Отдельный раздел посвящен случаю $\mathrm{SL}(2, \mathbb{R}) \cong \mathrm{SU}(1,1)$, в котором $P$ есть подгруппа треугольных матриц. $\mathrm{Y}$ всех этих групп имеется единственная, с точностью до сопряженности, максимальная параболическая подгруппа $P$, и эта подгруппа представима в виде полупрямого произведения: $P=\mathbb{R}_{+}^{*} \curlywedge P_{0}$. Тем самым, описание интегральных моделей по существу сводится к описанию канонических представлений подгруппы $P_{0}$.

Мы начинаем со случая подгруппы $P \subset \mathrm{O}(n, 1), n>2$, поскольку в этом случае имеется единственное, с точностью до сопряженности, каноническое представление подгруппы $P_{0}$ и соответственно единственная интегральная модель представления группы $P^{X}$.

5.1. Предварительные определения и обозначения. По определению, $\mathrm{O}(n, 1)$ есть группа линейных преобразований в $\mathbb{R}^{n+1}$, сохраняющих невырожденную квадратичную форму сигнатуры $(n, 1)$. Здесь в качестве такой формы выбрана квадратичная форма $2 x_{1} x_{n+1}+x_{2}^{2}+\cdots+x_{n}^{2}$, а элементы группы $\mathrm{O}(n, 1)$ записываются в виде блочных матриц

$$
g=\left\|g_{i j}\right\|_{i, j=1,2,3},
$$

где на диагонали стоят квадратные матрицы соответственно порядка $1, n-1$ и 1. 
Такая матричная реализация группы $\mathrm{O}(n, 1)$ удобна для описания ее максимальной параболической подгруппы $P \subset \mathrm{O}(n, 1)$, являющейся, по определению, подгруппой всех линейных преобразований, сохраняющих изотропное относительно введенной квадратичной формы подпространство $E$. С точностью до сопряженности, группа $\mathrm{O}(n, 1)$ обладает единственной максимальной параболической подгруппой. В принятой реализации изотропным подпространством $E$ является одномерное подпространство векторов $\left(x_{1}, 0, \ldots, 0\right)$, а соответствующая максимальная параболическая подгруппами $P$ группы $\mathrm{O}(n, 1)$ представима как подгруппа всех нижних клеточно-треугольных матриц в виде полупрямого произведения

$$
P=D<N,
$$

где $N \cong \mathbb{R}^{n-1}$ - максимальная нильпотентная подгруппа клеточных матриц вида

$$
h=\left(\begin{array}{ccc}
1 & 0 & 0 \\
-\gamma^{*} & e_{n-1} & 0 \\
-\frac{1}{2} \gamma \gamma^{*} & \gamma & 1
\end{array}\right), \quad \gamma \in \mathbb{R}^{n-1},
$$

а $D \cong \mathbb{R}^{*} \times \mathrm{O}(n-1)$ - подгруппа клеточно-диагональных матриц вида $d=$ $\operatorname{diag}\left(s^{-1}, u, s\right), s \in \mathbb{R}^{*}, u \in \mathrm{O}(n-1)$.

Будем представлять $D$ в виде прямого произведения $D=\mathbb{R}_{+}^{*} \times D_{0}$, где $D_{0}-$ подгруппа матриц $d=\operatorname{diag}( \pm 1, u, \pm 1)$, и обозначим:

$$
P_{0}=D_{0} \curlywedge N \text {. }
$$

Таким образом,

$$
P=\mathbb{R}_{+}^{*} \wedge P_{0}=\left(\mathbb{R}_{+}^{*} \times D_{0}\right) \curlywedge N .
$$

Элементы из $\mathbb{R}_{+}^{*}, D_{0}$ и $N$ будем обозначать соответственно через $r,(\varepsilon, u)$ (где $\varepsilon= \pm 1)$ и $\gamma$ (вектор-строка). В этих обозначениях групповые соотношения имеют вид:

$$
(\varepsilon, u)^{-1} g(\varepsilon, u)=\varepsilon \gamma u, \quad r g r^{-1}=r \gamma \quad \text { при } g=\gamma \in N .
$$

5.2. Описание канонических представлений подгруппы $P_{0}$. С точностью до сопряженности относительно группы автоморфизмов $\mathbb{R}_{+}^{*}$, существует единственное каноническое неприводимое унитарное представление $T$ подгруппы $P_{0}=D_{0} \curlywedge N$. Оно реализуется в гильбертовом пространстве $H$ функций на единичной сфере $S^{n-2} \subset \mathbb{R}^{n-1}$ с нормой

$$
\|f\|^{2}=\int_{S^{n-2}}|f(\omega)|^{2} d \omega
$$
где $d \omega$ - инвариантная мера на $S^{n-2}$, нормированная условием $\int_{S^{n-2}} d \omega=1$.
Операторы представления задаются формулами

$$
\begin{aligned}
(T(\gamma) f)(\omega) & =e^{-i\langle\gamma, \omega\rangle} f(\omega) \quad \text { при } \gamma \in N, N \cong \mathbb{R}^{n-1}, \\
(T(\varepsilon \omega u) f)(\omega) & =f(\varepsilon \omega u) \quad \text { при }(\varepsilon, u) \in D_{0}, D_{0}=\{ \pm 1\} \times \mathrm{O}(n-1) .
\end{aligned}
$$

Операторы $T_{r}, r \in \mathbb{R}_{+}^{*}$, представлений, сопряженных с $T$, действуют в пространствах $H_{r}=H$ и задаются формулами

$$
\left(T_{r}(\gamma) f\right)(\omega)=e^{-i r\langle\gamma, \omega\rangle} f(\omega), \quad T_{r}(g)=T(g) \quad \text { при } g \in D_{0} .
$$


ЗАмечание. Представление $T^{-}$группы $P_{0}$, заданное формулами

$$
\left(T^{-}(\gamma) f\right)(\omega)=e^{i\langle\gamma, \omega\rangle} f(\omega), \quad T^{-}(g)=T(g) \quad \text { при } g \in D_{0},
$$

эквивалентно представлению $T: T^{-}=A^{-1} T A$, где $A f(\omega)=f(-\omega)$.

ПРеДЛОЖЕНИЕ 5.1. Представление $T$ группъ $P_{0}$ канонично.

ДокАЗАТЕльство. Очевидно, что представления $T_{r}$ попарно неэквивалентны. Поэтому достаточно проверить оценку

$$
\left\|T_{r}(g) \mathbb{I}-\mathbb{I}\right\|<c(g) r \quad \text { для любого } g \in P_{0},
$$

где через $\mathbb{I}$ обозначен вектор $f(\omega) \equiv 1$. Поскольку $T_{r}(g) \mathbb{I}=\mathbb{I}$ при $g \in D_{0}$, то достаточно доказать (5.4) только для элементов $g=\gamma \in N$. Для этих элементов оценка следует из очевидного равенства

$$
\left\|T_{r}(g) \mathbb{I}-\mathbb{I}\right\|^{2}=2 \int_{S^{n-2}}(1-\cos (r\langle\gamma, \omega\rangle)) d \omega .
$$

5.3. Особое представление группы $P$. Ассоциированное с $T$ особое неприводимое представление $\widetilde{T}$ группы $P$ действует в прямом интеграле по мере $d^{*} r=r^{-1} d r$ на $\mathbb{R}_{+}^{*}$ гильбертовых пространств $H_{r}=H$,

$$
\mathscr{H}=\int_{0}^{\infty} H_{r} d^{*} r
$$

т. е. в пространстве сечений $f(r)$ расслоения над $\mathbb{R}_{+}^{*}$ со слоем $H_{r}$. Операторы подгруппы $P_{0}$ действуют в слоях этого расслоения, $(\widetilde{T}(g) f)(r)=T_{r}(g f(r))$ при $g \in P_{0}$, а операторы подгруппы $\mathbb{R}_{+}^{*}$ задаются формулой

$$
\left(\widetilde{T}\left(r_{0}\right) f\right)(r)=f\left(r_{0} r\right) .
$$

Нетривиальный 1-коцикл $b: P \rightarrow \mathscr{H}$, связанный с этим представлением, будем задавать в виде

$$
b(g)=\widetilde{T}(g) f_{0}(r, \omega)-f_{0}(r, \omega), \quad \text { где } f_{0}(r, \omega)=e^{-r / 2} .
$$

ПРЕДЛОЖЕНИЕ 5.2. Функиии $\|b(g)\|^{2} u c(g)=\left\langle b(g), f_{0}\right\rangle$ задаются следующими формулами:

$$
\begin{aligned}
\|b(g)\|^{2} & =\log \frac{\left(r_{0}+1\right)^{2}}{4 r_{0}}, \quad c(g)=\log \frac{2}{r_{0}+1} \quad \text { npu } g=r_{0} \in \mathbb{R}_{+}^{*} \\
\|b(g)\| & =2 c(g)=\int_{0}^{\pi / 2}\left(1+|\gamma|^{2} \cos ^{2} t\right) \sin ^{n-3} t d t \quad \text { npu } g=\gamma \in N .
\end{aligned}
$$

ДокАЗАТЕЛЬСтво. Воспользуемся равенством

$$
\int_{0}^{\infty}\left(e^{-a r}-e^{-b r}\right) r^{-1} d r=\log \left(\frac{b}{a}\right) \quad \text { при } \operatorname{Re} a, \operatorname{Re} b>0 .
$$


Имеем:

$$
\begin{aligned}
\left\|b\left(r_{0}\right)\right\|^{2} & =\int_{0}^{\infty} \int_{S^{n-2}}\left(e^{-r_{0} r}-2 e^{-\left(r_{0}+1\right) r / 2}+e^{-r}\right) d \omega d^{*} r \\
c(g) & =\int_{0}^{\infty} \int_{S^{n-2}}\left(e^{-\left(r_{0}+1\right) r / 2}-e^{-r}\right) d \omega d^{*} r .
\end{aligned}
$$

Отсюда на основании (5.8) следует (5.6).

Далее, имеем:

$$
\|b(\gamma)\|^{2}=\int_{0}^{\infty} \int_{S^{n-2}}\left(2 e^{-r}-e^{-(1+i\langle\gamma, \omega\rangle) r}-e^{-(1-i\langle\gamma, \omega\rangle) r}\right) d \omega d^{*} r .
$$

Интегрируя сначала по $r$, получаем на основании (5.8):

$$
\|b(\gamma)\|^{2}=\int_{S^{n-2}} \log \left(1+\langle\gamma, \omega\rangle^{2}\right) d \omega .
$$

Перейдя к сферическим координатам и проинтегрировав по $S^{n-3}$, получаем:

$$
\|b(\gamma)\|^{2}=\int_{0}^{\pi} \log \left(1+|\gamma|^{2} \cos ^{2} t\right) \sin ^{n-3} t d t .
$$

Аналогичным вычислением получаем выражение для $C(g)$.

5.4. Продолжение особого представления группы $P$ до представления группы $\mathrm{O}(n, 1)$. Для построения этого продолжения сначала опишем реализацию особого представления группы $\mathrm{O}(n, 1)$ в пространстве функций на $N \cong \mathbb{R}^{n-1}$; в дальнейшем элементы из $N$ будем отождествлять с точками $x \in \mathbb{R}^{n-1}$. Воспользовавшись разложением $\mathrm{O}(n, 1)=P^{+} N$, где $P^{+} \cong P$ подгруппа верхних клеточно-треугольных матриц, можно интерпретировать $N$ как сечение расслоения $\mathrm{O}(n, 1) \rightarrow P^{+} \backslash \mathrm{O}(n, 1)$. Тем самым, на $N$ определено действие $x \mapsto x g$ группы $\mathrm{O}(n, 1)$ :

$$
x g=\left(-\frac{|x|^{2}}{2} g_{13}+x g_{23}+g_{33}\right)^{-1}\left(-\frac{|x|^{2}}{2} g_{12}+x g_{22}+g_{32}\right),
$$

где $g_{i j}$ - элементы клеточной матрицы $g \in \mathrm{O}(n, 1)$. В частности,

$$
\begin{gathered}
x g=x+x_{0} \quad \text { при } g=x_{0} \in N ; \quad x g=\varepsilon^{-1} \gamma u \quad \text { при } g=\operatorname{diag}\left(\varepsilon^{-1}, u, \varepsilon\right) ; \\
x g=-\frac{2 x}{|x|^{2}} \quad \text { при } g=s=\left(\begin{array}{ccc}
0 & 0 & 1 \\
0 & e_{n-1} & 0 \\
1 & 0 & 0
\end{array}\right) .
\end{gathered}
$$

Определим далее функцию $\beta(x, g)$ формулой

$$
\beta(x, g)=\left|-\frac{|x|^{2}}{2} g_{13}+x g_{23}+g_{33}\right|, \quad x \in \mathbb{R}^{n-1}, \quad g \in \mathrm{O}(n, 1) .
$$

В частности, $\beta(x, g)=1$ при $g \in N ; \beta(x, g)=|\varepsilon|$ при $g=\operatorname{diag}\left(\varepsilon^{-1}, u, \varepsilon\right)$; $\beta(x, s)=|x|^{2} / 2$. 
ОПРЕДЕЛЕНИЕ 8 (см. [24]). Особое представление группы $\mathrm{O}(n, 1)$ реализуется в гильбертовом пространстве $\widetilde{\mathscr{H}}$ функций $\varphi(x)$ на $\mathbb{R}^{n-1}$, удовлетворяющих условию

$$
\int_{\mathbb{R}^{n-1}} \varphi(x) d x=0
$$

$\left(d x\right.$ - лебегова мера на $\left.\mathbb{R}^{n-1}\right)$, со скалярным произведением

$$
\left\langle\varphi_{1}, \varphi_{2}\right\rangle=-\int_{\mathbb{R}^{n-1} \times \mathbb{R}^{n-1}} \log \left|x^{\prime}-x^{\prime \prime}\right| \varphi_{1}\left(x^{\prime}\right) \overline{\varphi_{2}\left(x^{\prime \prime}\right)} d x^{\prime} d x^{\prime \prime} .
$$

Операторы представления имеют вид:

$$
(T(g) \varphi)(x)=\varphi(x g) \beta^{1-n}(x, g) .
$$

В частности,

$$
\begin{aligned}
& (T(g) \varphi)(x)=\varphi\left(x+x_{0}\right) \quad \text { при } g=x_{0} \in N \\
& (T(g) \varphi)(x)=|\varepsilon|^{1-n} \varphi\left(\varepsilon^{-1} \gamma u\right) \quad \text { при } g=\operatorname{diag}\left(\varepsilon^{-1}, u, \varepsilon\right) ; \\
& (T(g) \varphi)(x)=\varphi\left(-\frac{2 x}{|x|^{2}}\right)\left(\frac{|x|^{2}}{2}\right)^{1-n} \quad \text { при } g=s=\left(\begin{array}{ccc}
0 & 0 & 1 \\
0 & e_{n-1} & 0 \\
1 & 0 & 0
\end{array}\right) .
\end{aligned}
$$

Унитарность и групповое свойство этих операторов следует из соотношений:

$$
\beta\left(x, g_{1} g_{2}\right)=\beta\left(x, g_{1}\right) \beta\left(x g_{1}, g_{2}\right)
$$

для любых $x \in \mathbb{R}^{n-1}$ и $g_{1}, g_{2} \in \mathrm{O}(n, 1)$,

$$
d(x g)=\beta^{1-n}(x, g) d x
$$

для любого $g \in \mathrm{O}(n, 1)$ и

$$
\left|x^{\prime}-x^{\prime \prime}\right|^{2}=\left|x^{\prime} g-x^{\prime \prime} g\right|^{2} \beta\left(x^{\prime}, g\right) \beta\left(x^{\prime \prime}, g\right)
$$

для любых $x^{\prime}, x^{\prime \prime} \in \mathbb{R}^{n-1}$ и $g \in \mathrm{O}(n, 1)$. Нетривиальный 1-коцикл этого представления удобно задавать формулой

$$
b(g, x)=T(g) \varphi_{0}-\varphi_{0},
$$

где $\varphi_{0}(x)$ - преобразование Фурье функции $e^{-|\gamma| / 2}$ на $\mathbb{R}^{n-1} ;$ основание для такого выбора функции $\varphi_{0}(x)$ будет пояснено ниже.

Искомая реализация особого представления группы $\mathrm{O}(n, 1)$ получается переходом от функций $\varphi(x)$ к их преобразованию Фурье

$$
f(\gamma)=\int_{\mathbb{R}^{n-1}} \varphi(x) e^{i\langle\gamma, x\rangle} \varphi(x) d x .
$$

Нетрудно убедиться, что при этом преобразовании пространство $\widetilde{\mathscr{H}}$ преобразуется в гильбертово пространство функций на $\mathbb{R}^{n-1}$, где норма в сферических координатах на $\mathbb{R}^{n-1}$ имеет вид

$$
\|f\|^{2}=\int_{0}^{\infty} \int_{S^{n-2}}|f(r, \omega)|^{2} d \omega d^{*} r
$$


т. е. в пространство $\mathscr{H}$ особого представления группы $P$. Очевидно, далее, что в этой новой реализации операторы представления подгруппы $P$ имеют вид

$$
\begin{aligned}
& (\widetilde{T}(g) \varphi)(\gamma)=e^{-i\left\langle\gamma, \gamma_{0}\right\rangle} f(\gamma) \quad \text { при } g=\gamma_{0} \in N, \\
& (\widetilde{T}(g) \varphi)(\gamma)=f(\varepsilon \gamma u) \quad \text { при } g=\operatorname{diag}\left(\varepsilon^{-1}, u, \varepsilon\right),
\end{aligned}
$$

т. е. они совпадают с операторами исходного особого представления группы $P$. Таким образом, полученное представление группы $\mathrm{O}(n, 1)$ является искомым продолжением на $\mathrm{O}(n, 1)$ исходного особого представления группы $P$.

Очевидно, далее, что 1-коцикл в пространстве функций $\varphi(x)$, заданный формулой (5.19), преобразуется в 1-коцикл исходного представления $b(g)=$ $\widetilde{T}(g) f_{0}-f_{0}$, где $f_{0}(r)=e^{-r / 2}$.

Операторы полученного продолжения представления подгруппы $P$ на группу $\mathrm{O}(n, 1)$ представимы в интегральной форме:

$$
(\widetilde{T}(g) \varphi)(\gamma)=\int_{\mathbb{R}^{n-1}} A\left(\gamma, \gamma^{\prime}, g\right) f\left(\gamma^{\prime}\right) d \gamma^{\prime},
$$

где

$$
A\left(\gamma, \gamma^{\prime}, g\right)=\int_{\mathbb{R}^{n-1}} \exp \left(i\left(\langle\gamma, x\rangle-\left\langle\gamma^{\prime}, x g\right\rangle\right)\right) \beta^{1-n}(x, g) d x
$$

Эти выражения упрощаются только для элементов подгруппы $P$.

5.5. Описание интегральной модели INT $T$ представления группы токов $P^{X}$, ассоциированного с представлением $T$ группы $P_{0}$. В соответствии с общей конструкцией, представление INT $T$ группы $P^{X}$ реализуется в прямом интеграле по мере $\mathscr{L}$ гильбертовых пространств $\mathscr{H}_{\xi}$,

$$
\operatorname{INT} H=\int_{l_{+}^{1}(X)}^{\oplus} \mathscr{H}_{\xi} d \mathscr{L}(\xi)
$$

где $\mathscr{H}_{\xi}, \xi=\left\{r_{k}, x_{k}\right\},-$ счетные тензорные степени гильбертова пространства $H_{r}=H$ функций $f(\omega)$ на сфере $S^{n-2}$ со стабилизирующим вектором $f(\omega) \equiv 1$ :

$$
\mathscr{H}_{\xi}=\bigotimes_{k=1}^{\infty} H_{r_{k}}, \quad H_{r_{k}}=H
$$

Таким образом, элементами пространства INT $H$ являются сечения $F(\xi)$ расслоения над $l_{+}^{1}(X)$ со слоем $\mathscr{H}_{\xi}$.

Операторы $U(g), g \in P_{0}^{X}$, действуют в слоях $\mathscr{H}_{\xi}$ расслоения по формуле

$$
U(g(\cdot))=\bigotimes_{k=1}^{\infty} T_{r_{k}}\left(g\left(x_{k}\right)\right)
$$

Операторы $U\left(r_{0}(\cdot)\right), r_{0} \in \mathbb{R}^{X}$, задаются формулой

$$
\left(U\left(r_{0}(\cdot)\right) F\right)(\xi)=\exp \left(\frac{1}{2} \int_{X} \log r_{0}(x) d m(x)\right) F\left(r_{0}(\cdot) \xi\right) .
$$


5.6. Формула для сферической функции и связь представления INT $T$ группы $P^{X}$ с ее фоковским представлением. В соответствии с разделом 4, сферическая функция представления INT $T$ группы $P^{X}$ задается равенством

$$
\Psi(g)=\langle U(g) \Omega, \Omega\rangle, \quad \text { где } \Omega(\xi)=\bigotimes_{k=1}^{\infty}\left(e^{-r_{k} / 2} h_{r_{k}}\right) \quad \text { при } \xi=\left\{r_{k}, x_{k}\right\} .
$$

Теорема 5.1. Сферическая функиия представления INT T имеет вид:

$$
\Psi(g)=\exp \left(-\frac{1}{2} \int_{X}\|b(g(x))\|^{2} d m(x)\right),
$$

где $b(g)$ - 1-коиикл особого представления группы $\mathrm{O}(n, 1)$.

В самом деле, в случае $\mathrm{O}(n, 1)$ мы имеем $\operatorname{Im} c(g)=0$, а потому равенство (5.26) следует непосредственно из общей формулы (4.21) для сферической функции интегральной модели.

Согласно общей конструкции фоковских моделей, сферическая функция $\Phi(g)=\langle U(g) \operatorname{EXP} 0, \operatorname{EXP} 0\rangle$ фоковского представления группы $P^{X}$, ассоциированная с представлением $\widetilde{T}$ группы $P$ и 1 -коциклом $b$, задается той же формулой (5.26). Поэтому из теоремы 5.1 вытекает следующий результат.

СледствиЕ. Интегралъная модель INT T представления группь $P^{X}$ эквивалентна ее фоковскому представлению, ассочиированному с представлением $\widetilde{T}$ группы $P$ и 1-коциклом b. Сплетающий оператор этих представлений порожден отображением ииклических векторов $\Omega \mapsto \operatorname{EXP} 0$.

5.7. Продолжение интегральной модели представления группы $P^{X}$ до представления группы $\mathrm{O}(n, 1)^{X}$. Пусть $\widetilde{T}$ - описанное в п. 5.4 продолжение на $\mathrm{O}(n, 1)$ особого представления $\widetilde{T}$ группы $P$, и пусть $b(g)=\widetilde{T}(g) f_{0}-f_{0}$, где $f_{0}(r)=e^{-r / 2},-$ его нетривиальный 1-коцикл.

Согласно п. 4.6, продолжение представления INT $T$ группы $P^{X}$ в пространстве INT $H$ до представления группы $\mathrm{O}(n, 1)^{X}$ строится так. В пространстве INT $H$ вводится тотальное множество векторов $F_{g}, g \in \mathrm{O}(n, 1)^{X}$, вида

$$
F_{g}(\xi)=\exp \left(-\int_{X} c(g(x)) d m(x)\right) \bigotimes_{k=1}^{\infty}\left(\widetilde{T}\left(g\left(x_{k}\right)\right) f_{0}\right)\left(r_{k}\right) \quad \text { при } \xi=\left\{r_{k}, x_{k}\right\}
$$

где $c(g)=\left\langle b(g), f_{0}\right\rangle$. Отметим, что $\operatorname{Im} c(g)=0$.

Векторы $F_{g}$ принадлежат пространству INT $H$, причем

$$
\left\langle F_{g_{1}}, F_{g_{2}}\right\rangle=\exp \left(\int_{X} c\left(g_{1}(x), g_{2}(x)\right) d m(x)\right), \quad \text { где } c\left(g_{1}, g_{2}\right)=\left\langle b\left(g_{1}\right), b\left(g_{2}\right)\right\rangle \text {. }
$$

Определим действие операторов $U(g), g \in \mathrm{O}(n, 1)^{X}$, на множестве векторов $F_{g}$ следующим равенством:

$$
U(g) F_{g_{1}}=\exp \left(-\int_{X} \lambda\left(g(x), g_{1}(x)\right) d m(x)\right) F_{g g_{1}},
$$

где

$$
\lambda\left(g, g_{1}\right)=\frac{1}{2}\|b(g)\|^{2}+\left\langle\widetilde{T}(g) b\left(g_{1}\right), b(g)\right\rangle .
$$


Согласно п. 4.6, эти операторы сохраняют скалярные произведения $\left\langle F_{g_{1}}, F_{g_{2}}\right\rangle$ и порождают представление группы $\mathrm{O}(n, 1)^{X}$ в пространстве INT $H$, являющееся продолжением исходного представления группы $P^{X}$.

Поскольку $\operatorname{Im} c(g)=0$, это представление группы $\mathrm{O}(n, 1)^{X}$ является настоящим (непроективным).

\section{6. Интегральные модели представлений группы токов $\operatorname{SL}(2, \mathbb{R})^{X}$}

Рассматривается подгруппа $P \subset \mathrm{SL}(2, \mathbb{R})$ вещественных матриц $g=\left(\begin{array}{cc}\alpha^{-1} & 0 \\ \gamma & \alpha\end{array}\right)$. Ее элементы условимся задавать в форме $g=\varepsilon(r, \gamma)$, где $\varepsilon= \pm 1$ и $(r, \gamma)=$ $\left(\begin{array}{cc}r^{-1 / 2} & 0 \\ r^{1 / 2} \gamma & r^{1 / 2}\end{array}\right), r>0$. В этих обозначениях групповая операция на $P$ имеет вид:

$$
\left(r_{1}, \gamma_{1}\right)\left(r_{2}, \gamma_{2}\right)=\left(r_{1} r_{2}, r_{2}^{-1} \gamma_{1}+\gamma_{2}\right)
$$

Группа $P$ представима как полупрямое произведение коммутативных групп: $P=\mathbb{R}_{+}^{*} \wedge P_{0}$, где $P_{0}=\{ \pm 1\} \times \mathbb{R}$ - подгруппа элементов $\varepsilon(1, \gamma)$, a $\mathbb{R}_{+}^{*}$ - подгруппа пар $(r, 0), r>0$. Группа $\mathbb{R}_{+}^{*}$ действует на $P_{0}$ преобразованиями $\varepsilon(1, \gamma) \rightarrow$ $\varepsilon(r, 0)(1, \gamma)(r, 0)^{-1}=\varepsilon(1, r \gamma)$.

6.1. Канонические представления подгруппы $P_{0}$ и ассоциированные с ними представления группы $P$. С точностью до перехода к сопряженным представлениям, группа $P_{0}$ обладает одним каноническим неприводимым ортогональным представлением $T^{0}$ в двумерном пространстве $H^{0}$. При окомплексивании оно распадается в прямую сумму двух канонических унитарных представлений $T^{ \pm}$в пространствах $H^{ \pm} \cong \mathbb{C}$. При этом $H^{0}$ есть подпространство в $H^{+} \oplus H^{-}$векторов $(x, \bar{x}) \in \mathbb{C}^{2}$.

Операторы $T^{ \pm}(\varepsilon(1, \gamma))$ действуют умножением на $e^{ \pm i \gamma}$. Соответственно операторы $T_{r}^{ \pm}(\varepsilon(1, \gamma)), r \in \mathbb{R}_{+}^{*}$, сопряженных представлений действуют умножением на $e^{ \pm i r \gamma}$. Каноничность представлений $T^{ \pm}$следует из оценки $\left|e^{ \pm i r \gamma}-1\right| \sim$ $|\gamma| r$ при $r \rightarrow 0$.

С представлениями $T^{ \pm}$подгруппы $P_{0}$ ассоциированы особые неприводимые унитарные представления $\widetilde{T}^{ \pm}$группы $P$. Они действуют в комплексном гильбертовом пространстве $\mathscr{H}=\mathscr{H}^{ \pm}$функций $f(r)$ на полуоси $r>0$ с нормой

$$
\|f\|^{2}=\int_{0}^{\infty}|f(r)|^{2} d^{*} r, \quad d^{*} r=r^{-1} d r
$$

и задаются формулами: $\widetilde{T}^{ \pm}(\varepsilon)=\mathrm{id}$ (тривиальность операторов представления на центре группы),

$$
\left(\widetilde{T}^{ \pm}\left(r_{0}, r_{0} \gamma\right) f\right)(r)=e^{ \pm i r_{0} r \gamma} f\left(r_{0} r\right)
$$

В частности,

$$
\begin{aligned}
\left(\widetilde{T}^{ \pm}(1, \gamma) f\right)(r) & =e^{ \pm i r \gamma} f(r), \\
\left(\widetilde{T}^{ \pm}\left(r_{0}, 0\right) f\right)(r) & =f\left(r_{0} r\right), \quad r_{0} \in \mathbb{R}_{+}^{*} .
\end{aligned}
$$

Эти представления обладают нетривиальными 1 -коциклами $b^{ \pm}: P \rightarrow \mathscr{H}$, структуру которых мы обсудим в следующем пункте. 
С ортогональным каноническим представлением $T^{0}$ группы $P_{0}$ ассоциировано особое ортогональное представление $\widetilde{T}^{0}$ группы $P$ в пространстве $\mathscr{H}^{0} \subset$ $\mathscr{H}^{+} \oplus \mathscr{H}^{-}$функций $f(r): \mathbb{R}_{+}^{*} \rightarrow H^{0}$ с нормой

$$
\|f\|^{2}=\int_{0}^{\infty}\|f(r)\|^{2} d^{*} r .
$$

Операторы представления $\widetilde{T}^{0}(g)$ получаются ограничением на $\mathscr{H}^{0}$ операторов $\widetilde{T}^{+}(g) \oplus \widetilde{T}^{-}(g)$ в пространстве $\mathscr{H}^{+} \oplus \mathscr{H}^{-}$.

6.2. Продолжение представлений $\widetilde{T}^{ \pm}$и $\widetilde{T}^{0}$ группы $P$ до представлений группы $\mathrm{SL}(2, \mathbb{R})$. Введем всюду плотные инвариантные подпространства в пространствах особых представлений группы $P$. Конструкция искомых продолжений сведется тем самым к конструкции продолжений только на этих подпространствах.

Начнем со случая представления $\widetilde{T}^{+}$группы $P$ в пространстве $\mathscr{H}^{+}$.

Обозначим через $L$ верхнюю комплексную полуплоскость $(\operatorname{Im} z>0)$ с заданным на ней действием группы $\mathrm{SL}(2, \mathbb{R})$ :

$$
z \mapsto g z=\frac{\delta z+\gamma}{\beta z+\alpha} \quad \text { при } g=\left(\begin{array}{ll}
\alpha & \beta \\
\gamma & \delta
\end{array}\right)
$$

(плоскость Лобачевского); в частности, $g z=z$ при $g= \pm e$ (где $e-$ единица группы) и

$$
g z=r(z+\gamma) \quad \text { при } g=(r, \gamma) \in P .
$$

Поставим в соответствие каждой точке $z=u+i v \in L$ функцию $f_{z}(r)$ на правой полуоси:

$$
f_{z}(r)=e^{i r z}=e^{-r(v-i u)}, \quad \text { где } z=u+i v, v>0 .
$$

В частности,

$$
f_{z_{0}}(r)=e^{-r} \quad \text { при } z_{0}=i .
$$

ПРЕДЛОжЕниЕ 6.1. Функиии $f_{z_{1}}-f_{z_{2}}$ принадлежат пространству $\mathscr{H}^{+}$, причем скалярное произведение любой пары этих функиий задается следующей формулой:

$$
\left\langle f_{z_{1}}-f_{z_{2}}, f_{z_{1}^{\prime}}-f_{z_{2}^{\prime}}\right\rangle=\sum_{j, k=1,2}(-1)^{j+k-1} c\left(z_{j}, z_{k}^{\prime}\right),
$$

гдe

$$
c\left(z_{1}, z_{2}\right)=\log \left(-i\left(z_{1}-\bar{z}_{2}\right)\right)=\log \left(\left(v_{1}+v_{2}\right)+i\left(u_{1}-u_{2}\right)\right) \quad n p u z_{k}=u_{k}+i v_{k} .
$$

В частности,

$$
\left\|f_{z_{1}}-f_{z_{2}}\right\|^{2}=\log \frac{\left|z_{1}-\bar{z}_{2}\right|^{2}}{4 \operatorname{Im} z_{1} \operatorname{Im} z_{2}} .
$$

Здесь и в дальнейшем через $\log$ обозначена главная ветвь логарифма на плоскости с разрезом вдоль вещественной отрицательной полуоси такая, что $\log 1=0$. 
ДокАЗАТЕЛЬСтво. Из определения скалярного произведения в пространстве $\mathscr{H}^{+}$следует: если $z_{j}=u_{j}+i v_{j}, z_{j}^{\prime}=u_{j}^{\prime}+i v_{j}^{\prime}, j=1,2$, то

$\left\langle f_{z_{1}}-f_{z_{2}}, f_{z_{1}^{\prime}}-f_{z_{2}^{\prime}}\right\rangle=\int_{0}^{\infty}\left(\sum_{j, k=1,2}(-1)^{j+k} \exp \left(-r\left(\left(v_{1}+v_{2}\right)-i\left(u_{1}-u_{2}\right)\right)\right)\right) r^{-1} d r$.

Сходимость этого интеграла и формула (6.5) следуют из соотношения

$$
\int_{0}^{\infty}\left(e^{-a r}-e^{-b r}\right) r^{-1} d r=\log b-\log a \quad \text { при } \operatorname{Re} a, \operatorname{Re} b>0 .
$$

СлеДСТВИЕ. Имеет место равенство:

$$
\left\langle f_{z_{1}}-f_{z_{0}}, f_{z_{2}}-f_{z_{0}}\right\rangle=c\left(z_{1}\right)+\overline{c\left(z_{2}\right)}-c\left(z_{1}, z_{2}\right)-c\left(z_{0}\right),
$$

¿əe

$$
c(z)=c\left(z, z_{0}\right), \quad z_{0}=i .
$$

В частности,

$$
\left\|f_{z}-f_{z_{0}}\right\|^{2}=2 \operatorname{Re} c(g)-c(z, z)-c\left(z_{0}\right) .
$$

ЗАмечАниЕ. Выражение для $\left\|f_{z_{1}}-f_{z_{2}}\right\|^{2}$ представимо в виде

$$
\left\|f_{z_{1}}-f_{z_{2}}\right\|^{2}=2 \log \left[\cosh \frac{d\left(z_{1}, z_{2}\right)}{2}\right]
$$

где $d\left(z_{1}, z_{2}\right)$ - расстояние Лобачевского между $z_{1}$ и $z_{2}$.

В самом деле, преобразуем выражение $I=\frac{4 \operatorname{Im} z_{1} \operatorname{Im} z_{2}}{\left|z_{1}-\bar{z}_{2}\right|^{2}}$, воспользовавшись формулой $\tanh \frac{d\left(z_{1}, z_{2}\right)}{2}=\frac{\left|z_{1}-z_{2}\right|}{\left|z_{1}-\bar{z}_{2}\right|}$. Имеем:

$$
I=\frac{\left|z_{1}-\bar{z}_{2}\right|^{2}-\left|z_{1}-z_{2}\right|^{2}}{\left|z_{1}-\bar{z}_{2}\right|^{2}}=1-\tanh ^{2} \frac{d\left(z_{1}, z_{2}\right)}{2}=\cosh ^{-2} \frac{d\left(z_{1}, z_{2}\right)}{2}
$$

Отсюда следует (6.11).

ОПРЕДЕЛЕНИЕ 9. Обозначим через $M^{+}$предгильбертово подпространство в $\mathscr{H}^{+}$, линейно порожденное функциями $f_{z_{1}}-f_{z_{2}}$ или, что эквивалентно, функциями $f_{z}-f_{z_{0}}$.

Из (6.4) вытекает следующее утверждение.

ПРЕДЛОЖЕНИЕ 6.2. Подпространство $M^{+}$инвариантно относительно действия операторов группы $P$, а именно, для любых $z_{1}, z_{2} \in L$ имеем:

$$
\widetilde{T}^{+}(g)\left(f_{z_{1}}-f_{z_{2}}\right)=f_{g z_{1}}-f_{g z_{2}} .
$$

Очевидно также, что подпространство $M^{+}$всюду плотно в $\mathscr{H}^{+}$. Поэтому для продолжения представления $T^{+}$группы $P$ до представления группы $\mathrm{SL}(2, \mathbb{R})^{X}$ достаточно определить действие операторов представления только на этом подпространстве. 
ОпредЕлЕниЕ 10. Определим операторы $\widetilde{T}^{+}$представления всей группы $\mathrm{SL}(2, \mathbb{R})$ на элементах пространства $M^{+}$той же формулой $(6.12)$.

Теорема 6.1. Операторы $\widetilde{T}^{+}(g), g \in \mathrm{SL}(2, \mathbb{R})$, сохраняют скалярное произведение на $M^{+}$и удовлетворяют групповому соотношению. Тем самым, они порождают продолжение представления $\widetilde{T}^{+}$группы $P$ до унитарного представления группы $\mathrm{SL}(2, \mathbb{R})$.

ДокАЗАТЕЛЬСтво. Групповое соотношение для этих операторов очевидно. Далее, из соотношения

$$
g z_{1}-g \bar{z}_{2}=\left(z_{1}-\bar{z}_{2}\right)\left(\beta z_{1}+\alpha\right)^{-1}\left(\beta \bar{z}_{2}+\alpha\right)^{-1} \quad \text { для любого } g=\left(\begin{array}{ll}
\alpha & \beta \\
\gamma & \delta
\end{array}\right)
$$

следует, что

$$
\sum_{j, k=1,2}(-1)^{j+k-1} c\left(g z_{j}, g z_{k}^{\prime}\right)=\sum_{j, k=1,2}(-1)^{j+k-1} c\left(z_{j}, z_{k}^{\prime}\right) \quad \text { для любого } g \in \operatorname{SL}(2, \mathbb{R}),
$$

т. е. скалярное произведение на $M^{+}$инвариантно относительно операторов $\widetilde{T}^{+}(g)$ группы $\mathrm{SL}(2, \mathbb{R})$.

ПредлОЖЕНИЕ 6.3. Представление $\widetilde{T}^{+}$группљ $\mathrm{SL}(2, \mathbb{R})$ обладает нетривиальным 1-коциклом $b^{+}: \mathrm{SL}(2, \mathbb{R}) \rightarrow \mathscr{H}^{+}$вида

$$
b^{+}(g)=f_{g z_{0}}-f_{z_{0}}, \quad \text { əде } z_{0}=i .
$$

В самом деле, $b^{+}(g) \in \mathscr{H}^{+}$при любом $g \in \mathrm{SL}(2, \mathbb{R})$. Далее, поскольку $f_{g z_{0}}=$ $\widetilde{T}^{+}(g) f_{z_{0}}$, то $b^{+}(g)=\widetilde{T}^{+}(g) f_{z_{0}}-f_{z_{0}}$, а потому $b^{+}(g)$ есть 1-коцикл. Так как $f_{z_{0}} \notin \mathscr{H}^{0}$, то этот 1-коцикл нетривиален.

Из формулы для скалярного произведения в $H$ вытекает следующее утверждение.

ПреДЛОЖЕНИЕ 6.4. Для любых $g_{1}, g_{2} \in \mathrm{SL}(2, \mathbb{R})$ справедливо равенство

$$
\left\langle b\left(g_{1}\right), b\left(g_{2}\right)\right\rangle=c\left(g_{1} z_{0}\right)+\overline{c\left(g_{2} z_{0}\right)}-c\left(g_{1} z_{0}, g_{2} z_{0}\right)-c\left(z_{0}, z_{0}\right) ;
$$

в частности,

$$
\|b(g)\|^{2}=2 \operatorname{Re} c\left(g z_{0}\right)-c\left(g z_{0}, g z_{0}\right)-c\left(z_{0}, z_{0}\right) .
$$

СлЕДСТВИЕ. Имеет место соотношение:

$$
\begin{gathered}
-\frac{1}{2}\|b(g)\|^{2}-\left\langle\widetilde{T}^{+}(g) b\left(g_{1}\right), b(g)\right\rangle=i \operatorname{Im} c\left(g z_{0}\right)+\left(c\left(g g_{1} z_{0}, g z_{0}\right)-c\left(g g_{1} z_{0}, z_{0}\right)\right) \\
-\frac{1}{2}\left(c\left(g z_{0}, g z_{0}\right)-c\left(z_{0}, z_{0}\right)\right) .
\end{gathered}
$$

Аналогично строится продолжение представления $\widetilde{T}^{-}$группы $P$ до унитарного представления группы $\operatorname{SL}(2, \mathbb{R})$. Именно, мы заменяем пространство $M^{+}$ функций $f_{z_{1}}-f_{z_{2}}$ на пространство $M^{-}$функций $\overline{f_{z_{1}}}-\overline{f_{z_{2}}}$. Очевидно, что $M^{-}$ тотально в $\mathscr{H}^{-}$и инвариантно относительно действия операторов группы $P$ :

$$
\widetilde{T}^{-}(g)\left(\overline{f_{z_{1}}}-\overline{f_{z_{2}}}\right)=\overline{f_{g z_{1}}}-\overline{f_{g z_{2}}} .
$$


Той же формулой (6.18) определяется продолжение представления $\widetilde{T}^{-}$подгруппы $P$ на всю группу $\mathrm{SL}(2, \mathbb{R})$. Нетривиальный 1 -коцикл $b^{-}$полученного представления связан с 1-коциклом $b^{+}$соотношением $b^{-}(g)=\overline{b^{+}(g)}$.

Определенные так продолжения представлений $\widetilde{T}^{ \pm}$подгруппы $P$ до унитарных представлений группы $\mathrm{SL}(2, \mathbb{R})$ индуцируют продолжение ортогонального представления подгруппы $P$ в пространстве $\mathscr{H}^{0} \subset \mathscr{H}^{+} \oplus \mathscr{H}^{-}$до ортогонального представления группы $\mathrm{SL}(2, \mathbb{R})$. Нетривиальный 1-коцикл, связанный с этим представлением, имеет вид:

$$
b^{0}(g)=\left(b^{+}(g), b^{-}(g)\right)
$$

6.3. Интегральные модели представлений группы токов $P^{X}$, ассоциированные с каноническими представлениями группы $P_{0}$. Поскольку пространства $H_{r}^{ \pm}$представлений $T_{r}^{ \pm}$группы $P_{0}$ одномерны, их счетные тензорные произведения $H_{\xi}^{ \pm}, \xi=\left\{r_{k}, x_{k}\right\}$, также одномерны. Таким образом, представления INT $T^{ \pm}$группы $P^{X}$ действуют в прямых интегралах по мере $\mathscr{L}$ одномерных пространств $H_{\xi}^{ \pm}$, т. е. в гильбертовых пространствах INT $H^{ \pm}$комплекснозначных функционалов $F^{ \pm}(\xi)=F\left(\left\{r_{k}, x_{k}\right\}\right)$ на $l_{+}^{1}(X)$ с нормой

$$
\|F\|^{2}=\int_{l_{+}^{1}(X)}|F(\xi)|^{2} d \mathscr{L}(\xi) .
$$

Операторы представления группы $P^{X}$ тождественны на ее центре и однозначно определяются формулами:

$$
\begin{aligned}
\left(U^{ \pm}(1, \gamma(\cdot)) F^{ \pm}\right)(\xi) & =\exp \left( \pm i \sum r_{k} \gamma\left(x_{k}\right)\right) F^{ \pm}(\xi) \\
\left(U^{ \pm}\left(r_{0}(\cdot), 0\right) F\right)(\xi) & =\exp \left(\frac{1}{2} \int_{X} \log r_{0}(x) d m(x)\right) F^{ \pm}\left(r_{0}(\cdot) \xi\right)
\end{aligned}
$$

при $\xi=\left\{r_{k}, x_{k}\right\}$.

Перейдем к описанию ортогонального представления INT $T^{0}$ группы $P^{X}$, acсоциированного с каноническим представлением $T^{0}$ подгруппы $P_{0}$. Здесь элементы пространств $H_{r}^{0}=H^{0}$ представлений $T_{r}^{0}$ - векторы $(s, \bar{s}), s \in \mathbb{C}$, а потому пространства $H_{\xi}^{0}$ представлений группы $P_{0}^{X}$ являются счетными тензорными произведениями двумерных вещественных пространств со стабилизирующим вектором $2^{-1 / 2}(1,1)$. Очевидно, что $H_{\xi}^{0} \subset H_{\xi}^{0} \otimes H_{\xi}^{0}$.

Таким образом, представление INT $T^{0}$, ассоциированное с каноническим представлением $T^{0}$ подгруппы $P_{0}$, реализуется в вещественном ортогональном пространстве INT $H^{0}$ функционалов $F(\xi)$ на $l_{+}^{1}(X)$ со значениями в пространствах $H_{\xi}^{0}$ с нормой

$$
\|F\|^{2}=\int_{l_{+}^{1}(X)}\|F(\xi)\|^{2} d \mathscr{L}(\xi) .
$$

Операторы $U^{0}(1, \gamma(\cdot))$ задаются равенством

$$
U^{0}(1, \gamma(\cdot))\left(\bigotimes_{k=1}^{\infty}\left(s_{k}, \overline{s_{k}}\right)\right)=\bigotimes_{k=1}^{\infty}\left(e^{i r_{k}^{2} \gamma\left(x_{k}\right)} s_{k}, e^{-i r_{k}^{2} \gamma\left(x_{k}\right)} \overline{s_{k}}\right)
$$


а операторы $U^{0}\left(r_{0}(\cdot), 0\right)$ задаются, как и в случае представлений $U^{ \pm}$, равенствами (6.20).

Заметим, что при естественном вложении

$$
\mathrm{INT} H^{0} \subset \operatorname{INT} H^{+} \otimes \operatorname{INT} H^{-}
$$

операторы $U^{0}(g)$ являются ограничениями на INT $H^{0}$ операторов $U^{+}(g) \otimes U^{-}(g)$ в пространстве INT $H^{+} \otimes \mathrm{INT} H^{-}$.

6.4. Продолжение унитарных представлений $U^{ \pm}=\operatorname{INT} T^{ \pm}$группы токов $P^{X}$ до проективных унитарных представлений группы $\mathrm{SL}(2, \mathbb{R})^{X}$. Построим продолжение унитарного представления $U^{+}=\operatorname{INT} T^{+}$группы токов $P^{X}$ в пространстве $U^{+}=\mathrm{INT} H^{+}$до унитарного проективного представления группы $\mathrm{SL}(2, \mathbb{R})^{X}$. Подобно случаю группы коэффициентов $\operatorname{SL}(2, \mathbb{R})$, действие операторов группы токов $\mathrm{SL}(2, \mathbb{R})^{X}$ будет определено на некотором тотальном подмножестве $\widetilde{M}^{+} \subset \mathrm{INT} H^{+}$.

Обозначим через $L^{X}$, где $L$ - верхняя комплексная полуплоскость, пространство ограниченных функций $z: X \rightarrow L, z(x)=u(x)+i v(x), v(x)>0$. Действие группы $\mathrm{SL}(2, \mathbb{R})$ на $L$ индуцирует поточечное действие на $L^{X}$ ее группы токов $\mathrm{SL}(2, \mathbb{R})^{X}$.

Поставим в соответствие каждой функции $z \in L^{X}$ следующий функционал $F_{z}(\xi)=F_{z}\left(\left\{r_{k}, x_{k}\right\}\right)$ на $l_{+}^{1}(X)$ :

$$
F_{z}^{+}(\xi)=\exp \left(i \sum r_{k} z\left(x_{k}\right)\right)=\exp \left(-\sum r_{k}\left(v\left(x_{k}\right)-i u\left(x_{k}\right)\right)\right) \quad \text { при } z=u+i v .
$$

Из определения характеристического функционала меры $\mathscr{L}$ следует, что

$$
\left\langle F_{z_{1}}^{+}, F_{z_{2}}^{+}\right\rangle=\exp \left(-\int_{X} c\left(z_{1}(x), z_{2}(x)\right) d m(x)\right)
$$

где функция $c\left(z_{1}, z_{2}\right)$ задается равенством (6.6). В частности,

$$
\left\|F_{z}^{+}\right\|^{2}=\exp \left(-\int_{X} \log (2 v(x)) d m(x)\right) \quad \text { при } z=u+i v .
$$

В силу условия ограниченности функций $z \in L^{X}$, функционалы $F_{z}^{+}$принадлежат пространству INT $H^{+}$, и легко убедиться, что множество этих функционалов тотально в INT $H^{+}$.

ОПРЕДЕЛЕНИЕ 11. Определим действие операторов $U^{+}(g)$ группы токов $\mathrm{SL}(2, \mathbb{R})^{X}$ на множестве функционалов $F_{z}^{+}$следующей формулой:

$$
U^{+}(g) F_{z}^{+}=\exp \left(\int_{X} \varphi(g(x), z(x)) d m(x)\right) F_{g z}^{+},
$$

где

$$
\varphi(g, z)=c\left(g z, g z_{0}\right)-c\left(z, z_{0}\right)-\frac{1}{2}\left(c\left(g z_{0}, g z_{0}\right)-c\left(z_{0}, z_{0}\right)\right), \quad z_{0}=i .
$$


ПРедЛОЖЕниЕ 6.5. На элементах подгруппъ $P^{X}$ операторъ $U^{+}(g)$ совпадают с операторами исходного представления группы $P^{X}$.

ДоказАтельство. При $g=\left(r_{0}, \gamma\right) \in P$ имеем: $c\left(g z_{1}, g z_{2}\right)=\log r_{0}+c\left(z_{1}, z_{2}\right)$. Отсюда следует, что множитель в формуле (6.24) для $U^{+}(g)$ равен единице при $g \in P_{0}$ и равен $\exp \left(\frac{1}{2} \int_{X} \log r_{0}(x) d m(x)\right)$ при $g=\left(r_{0}(\cdot), \gamma(\cdot)\right) \in P^{X}$. Далее, имеем: $F_{g z}^{+}(\xi)=\exp \left(i \sum r_{k} \gamma\left(x_{k}\right)\right) F_{z}^{+}(\xi)$ при $g=(1, \gamma(\cdot))$ и $F_{g z}^{+}(\xi)=F_{z}^{+}\left(r_{0}(\cdot) \xi\right)$ при $g=\left(r_{0}(\cdot), 0\right)$. Следовательно,

$$
\begin{aligned}
U^{+}(g) F_{z}^{+}(\xi) & =\exp \left(i \sum r_{k} \gamma\left(x_{k}\right)\right) F_{z}^{+}(\xi) \quad \text { при } g=(1, \gamma(\cdot)), \\
U^{+}(g) F_{z}^{+}(\xi) & =\exp \left(\frac{1}{2} \int_{X} \log r_{0}(x) d m(x)\right) F_{z}^{+}\left(r_{0}(\cdot) \xi\right) \quad \text { при } g=\left(r_{0}(\cdot), 0\right)
\end{aligned}
$$

для любого $z \in L^{X}$. Отсюда следует утверждение.

Убедимся, что операторы $U^{+}$задают продолжение представления группы $P^{X}$ на всю группу $\mathrm{SL}(2, \mathbb{R})^{X}$. Для этого заменим множество функционалов $F_{z}^{+}$на множество $\widetilde{M}^{+}$функционалов вида

$$
\Psi_{g}^{+}=2^{-1 / 2} \exp \left(\int_{X} c(z(x)) d m(x)\right) F_{z}, \quad g \in \mathrm{SL}(2, \mathbb{R})^{X}, \quad \text { где } z=g z_{0} .
$$

ПреДложениЕ 6.6. На множестве $\widetilde{M}^{+}$скалярное произведение и операторы представления задаются формулами:

$$
\left\langle\Psi_{g_{1}}^{+}, \Psi_{g_{2}}^{+}\right\rangle=\exp \left(\int_{X}\left\langle b\left(g_{1}(x)\right), b\left(g_{2}(x)\right)\right\rangle d m(x)\right),
$$

где $b(g)-1$-коцикл $P \rightarrow \mathscr{H}^{+}$, заданный равенством (6.14);

$$
U^{+}\left(g_{1}\right) \Psi_{g}^{+}=\exp \left(-\int_{X} u\left(g_{1}(x), g(x)\right) d m(x)\right) \Psi_{g_{1} g}^{+},
$$

где

$$
u\left(g_{1}, g\right)=i \operatorname{Im} c\left(g_{1} z_{0}\right)+\frac{1}{2}\left\|b\left(g_{1}\right)\right\|^{2}+\left\langle\widetilde{T}\left(g_{1}\right) b(g), b\left(g_{1}\right)\right\rangle .
$$

ДоказАтельство. Равенство (6.26) следует из соотношения (6.15). Равенство (6.27) следует из соотношения (6.17), в котором следует поменять местами $g$ и $g_{1}$.

ТеОрема 6.2. Операторы $U^{+}(g)$ сохраняют скалярные произведения $\left\langle\Psi_{g_{1}}^{+}, \Psi_{g_{2}}^{+}\right\rangle$, а потому продолжаются до унитарных операторов на всем пространстве INT $H^{+}$.

ДоказАтельство. Имеем:

$$
\left\langle U^{+}(g) \Psi_{g_{1}}^{+}, U^{+}(g) \Psi_{g_{2}}^{+}\right\rangle=\exp \left(\int_{X} v\left(g(x), g_{1}(x), g_{2}(x)\right) d m(x)\right),
$$


где

$$
v\left(g, g_{1}, g_{2}\right)=-\left(u\left(g, g_{1}\right)+\overline{u\left(g, g_{2}\right)}\right)+\left\langle b\left(g g_{1}\right), b\left(g g_{2}\right)\right\rangle .
$$

Отсюда и из равенства

$\left\langle b\left(g g_{1}\right), b\left(g g_{2}\right)\right\rangle=\|b(g)\|^{2}+\left\langle\widetilde{T}^{+}(g) b\left(g_{1}\right), b(g)\right\rangle+\left\langle b(g), \widetilde{T}^{+}(g) b\left(g_{2}\right)\right\rangle+\left\langle b\left(g_{1}\right), b\left(g_{2}\right)\right\rangle$ следует, что $\left\langle U^{+}(g) \Psi_{g_{1}}^{+}, U^{+}(g) \Psi_{g_{2}}^{+}\right\rangle=\exp \left(\int_{X}\left\langle b\left(g_{1}(x)\right), b\left(g_{2}(x)\right)\right\rangle d m(x)\right)$.

Теорема 6.3. Операторы $U^{+}$образуют проективное представление группъ $\mathrm{SL}(2, \mathbb{R})^{X} ;$ именно, для любых $g_{1}, g_{2} \in \mathrm{SL}(2, \mathbb{R})^{X}$ имеем:

$$
U^{+}\left(g_{1} g_{2}\right)=\exp \left(i \operatorname{Im} \int_{X} a\left(g_{1}(x), g_{2}(x)\right) d m(x)\right) U^{+}\left(g_{1}\right) U^{+}\left(g_{2}\right),
$$

где

$$
a\left(g_{1}, g_{2}\right)=c\left(g_{1} z_{0}\right)+c\left(g_{2} z_{0}\right)-c\left(g_{1} g_{2} z_{0}\right)+\left\langle T\left(g_{1}\right) b\left(g_{2}\right), b\left(g_{1}\right)\right\rangle .
$$

ДокАЗАТЕЛЬСтво. Согласно (6.27), имеем:

$$
\begin{aligned}
U^{+}\left(g_{1}\right) U^{+}\left(g_{2}\right) \Psi_{g}^{+} & =\exp \left(-\int_{X} a_{1}\left(g_{1}(x), g_{2}(x)\right) d m(x)\right) \Psi_{g_{1} g_{2} g}^{+}, \\
U^{+}\left(g_{1} g_{2}\right) \Psi_{g}^{+} & =\exp \left(-\int_{X} a_{2}\left(g_{1} g_{2}(x)\right) d m(x)\right) F_{g_{1} g_{2} g}^{+}
\end{aligned}
$$

где

$$
a_{1}\left(g_{1}, g_{2}\right)=u\left(g_{2}, g\right)+u\left(g_{1}, g_{2} g\right), \quad a_{2}\left(g_{1} g_{2}\right)=u\left(g_{1} g_{2}, g\right) .
$$

Следовательно,

$$
U^{+}\left(g_{1}\right) U^{+}\left(g_{2}\right) \Psi_{g}=\exp \left(-\int_{X} a^{\prime}\left(g_{1}(x), g_{2}(x)\right) d m(x)\right) U^{+}\left(g_{1} g_{2}\right) \Psi_{g},
$$

где

$$
a^{\prime}\left(g_{1}, g_{2}\right)=u\left(g_{2}, g\right)+u\left(g_{1}, g_{2} g\right)-u\left(g_{1} g_{2}, g\right),
$$

т. е., согласно (6.28),

$a^{\prime}\left(g_{1}, g_{2}\right)=i \operatorname{Im}\left(c\left(g_{1} z_{0}\right)+c\left(g_{2} z_{0}\right)-c\left(g_{1} g_{2} z_{0}\right)\right)+v\left(g_{2}, g\right)+v\left(g_{1}, g_{2} g\right)-v\left(g_{1} g_{2}, g\right)$, где $v\left(g_{1}, g\right)=\frac{1}{2}\left\|b\left(g_{1}\right)\right\|^{2}+\left\langle\widetilde{T}^{+}\left(g_{1}\right) b(g), b\left(g_{1}\right)\right\rangle$. Отсюда и из соотношения

$$
v\left(g_{2}, g\right)+v\left(g_{1}, g_{2} g\right)-v\left(g_{1} g_{2}, g\right)=i \operatorname{Im}\left\langle\widetilde{T}^{+}\left(g_{1}\right) b\left(g_{2}\right), b\left(g_{1}\right)\right\rangle
$$

следует, что

$$
a^{\prime}\left(g_{1}, g_{2}\right)=i \operatorname{Im}\left(c\left(g_{1} z_{0}\right)+c\left(g_{2} z_{0}\right)-c\left(g_{1} g_{2} z_{0}\right)+\left\langle\widetilde{T}^{+}\left(g_{1}\right) b\left(g_{2}\right), b\left(g_{1}\right)\right\rangle\right) .
$$

Таким образом,

$$
\begin{aligned}
& U^{+}\left(g_{1} g_{2}\right) \Psi_{g} \\
& \quad=\exp \left(i \operatorname{Im} \int_{X} a\left(g_{1}(x), g_{2}(x)\right) d m(x)\right) U^{+}\left(g_{1}\right) U^{+}\left(g_{2}\right) \Psi_{g} \text { для любого } g \in P^{X},
\end{aligned}
$$

где $a\left(g_{1}, g_{2}\right)$ задается равенством (6.30) и не зависит от $g$. Отсюда следует (6.29). Теорема 6.3 доказана. 
Продолжение второго представления $U^{-}$группы $P^{X}$ до представления группы $\mathrm{SL}(2, \mathbb{R})^{X}$ получается заменой тотального множества $\widetilde{M}^{+} \subset \widetilde{H}^{+}$тотальным множеством $\widetilde{M}^{-} \subset \widetilde{H}^{-}$функционалов $\Psi_{g}^{-}=\overline{\Psi_{g}^{+}}$. При этом формулы для скалярных произведений $\left\langle\Psi_{g_{1}}^{-}, \Psi_{g_{2}}^{-}\right\rangle$и для операторов $U^{-}(g)$, а также соотношение между операторами $U^{-}\left(g_{1} g_{2}\right)$ и $U^{-}\left(g_{1}\right) U^{-}\left(g_{2}\right)$ получаются из соответствующих формул $(6.26),(6.27)$ и $(6.29)$ для случая $U^{+}$комплексным сопряжением:

$$
\begin{aligned}
\left\langle\Psi_{g_{1}}^{-}, \Psi_{g_{2}}^{-}\right\rangle & =\exp \left(\int_{X}\left\langle b\left(g_{2}(x)\right), b\left(g_{1}(x)\right)\right\rangle d m(x)\right) \\
U^{-}\left(g_{1}\right) \Psi_{g}^{-} & =\exp \left(-\int_{X} \overline{u\left(g_{1}(x), g(x)\right)} d m(x)\right) \Psi_{g_{1} g}^{-}, \\
U^{-}\left(g_{1} g_{2}\right) & =\exp \left(-i \operatorname{Im} \int_{X} a\left(g_{1}(x), g_{2}(x)\right) d m(x)\right) U^{-}\left(g_{1}\right) U^{-}\left(g_{2}\right) .
\end{aligned}
$$

6.5. Продолжение ортогонального представления $U^{0}=\operatorname{INT} T^{0}$ группы токов $P^{X}$ до ортогонального представления группы $\operatorname{SL}(2, \mathbb{R})^{X}$. Kaк отмечалось, при естественном вложении INT $H^{0} \subset \mathrm{INT} H^{+} \otimes \mathrm{INT} H^{-}$операторы $U^{0}(g)$ группы $P^{X}$ получаются ограничением на INT $H^{0}$ операторов $U^{+}(g) \otimes$ $U^{-}(g)$ в пространстве INT $H^{+} \otimes \mathrm{INT} H^{-}$. Тем самым, построенные продолжения представлений $U^{ \pm}$группы $P^{X}$ до представлений группы $\operatorname{SL}(2, \mathbb{R})^{X}$ индуцируют продолжение представления $U^{0}$ группы $P^{X}$ в пространстве INT $H^{+}$до ортогонального представления группы $\operatorname{SL}(2, \mathbb{R})^{X}$. Его окомплексивание есть унитарное непроективное представление $\operatorname{INT}\left(T^{+} \oplus T^{-}\right)=\operatorname{INT} T^{+} \otimes \operatorname{INT} T^{-}$, эквивалентное представлениям группы $\mathrm{SL}(2, \mathbb{R})$, построенного ранее в [1].

Приведем независимое описание продолжения представления $U^{0}(g)$ группы $P^{X}$ до представления группы $\mathrm{SL}(2, \mathbb{R})^{X}$.

Отнесем каждой паре $z \in L^{X}$ и $(r, x) \in \mathbb{R}_{+}^{*} \times X$ вектор $f_{z, r, x}^{0} \in H_{r}^{0}$,

$$
f_{z, r, x}^{0}=2^{-1 / 2}\left(e^{i r_{k} z\left(x_{k}\right)}, e^{-i r_{k} \overline{z\left(x_{k}\right)}}\right),
$$

и определим функционалы $F_{z}^{0}(\xi), z \in L^{X}$, на $l_{+}^{1}(X)$ следующим равенством:

$$
F_{z}^{0}(\xi)=\bigotimes_{k=1}^{\infty} f_{z, r_{k}, x_{k}}^{0}
$$

Теорема 6.4. Для любъх $z_{1}, z_{2} \in L^{X}$ имеем

$$
\begin{aligned}
\left\langle F_{z_{1}}^{0}, F_{z_{2}}^{0}\right\rangle & =\exp \left(-\int_{X} \log \left|z_{1}(x)-\overline{z_{2}(x)}\right| d m(x)\right) \\
& =\exp \left(-\int_{X} \operatorname{Re} c\left(z_{1}(x), z_{2}(x)\right) d m(x)\right) .
\end{aligned}
$$

В частности,

$$
\left\|F_{z}^{0}\right\|^{2}=\exp \left(-\int_{X} \log (2 \operatorname{Im} z(x)) d m(x)\right) .
$$

ДокАЗАТЕЛьство. Заметим, что для

$$
\left\langle f_{z_{1}, r, x}^{0}, f_{z_{2}, r, x}^{0}\right\rangle=\frac{1}{2}\left(e^{i r z(x)}, e^{-i r \overline{z(x)}}\right), \quad \text { где } z=z_{1}-\overline{z_{2}} \quad \text { для любых } z_{1}, z_{2} \in L^{X} .
$$


Следовательно,

$$
\left\langle F_{z_{1}}^{0}, F_{z_{2}}^{0}\right\rangle=\int_{l_{+}^{1}(X)} \prod_{k=1}^{\infty}\left(\frac{1}{2} e^{i r_{k} z\left(x_{k}\right)}+\frac{1}{2} e^{-i r_{k} \bar{z}\left(x_{k}\right)}\right) d \mathscr{L}(\xi), \quad \text { где } z=z_{1}-z_{2} .
$$

Для вычисления интеграла воспользуемся теоремой 2.3. Имеем на основании этой теоремы:

$$
\left\langle F_{z_{1}}^{0}, F_{z_{2}}^{0}\right\rangle=\exp \left(\int_{X} \int_{0}^{\infty}\left(\frac{1}{2} e^{i r z(x)}+\frac{1}{2} e^{-i r \bar{z}(x)}-e^{-r}\right) r^{-1} d r d m(x)\right),
$$

т. e.

$$
\left\langle F_{z_{1}}^{0}, F_{z_{2}}^{0}\right\rangle=\exp \left(\frac{1}{2} \int_{X}(a(x)+\overline{a(x)}) d m(x)\right)
$$

где

$$
a(x)=\int_{0}^{\infty}\left(e^{i r z(x)}-e^{-r}\right) r^{-1} d r .
$$

Поскольку $e^{i r z(x)}=e^{-r(v(x)-i u(x))}$ при $z=u+i v$, где $v>0$, то

$$
a(x)=-\log (v(x)-i u(x))
$$

Отсюда следует (6.35).

СлЕДСТВиЕ. Функиионалы $F_{z}^{0}$ принадлежат пространству INT $H^{0}$.

Легко убедиться, что множество этих функционалов тотально в INT $H^{0}$.

ОПРЕДЕлЕниЕ 12. Зададим операторы $U^{0}(g), g \in \mathrm{SL}(2, \mathbb{R})^{X}$, на $\widetilde{M}^{0}$ следующим равенством:

$$
U^{0}(g) F_{z}^{0}=\exp \left(\operatorname{Re} \int_{X} \varphi(g(x), z(x)) d m(x)\right) F_{g z}^{0}
$$

где $\varphi(g, z)$ задается равенством (6.25). Другими словами, формула для $U^{0}(g)$ получается из формулы для $U^{ \pm}(g)$ заменой функции $\varphi(g, z)$ ее вещественной частью.

Подобно операторам $U^{ \pm}$, на элементах подгруппы $P^{X}$ эти операторы совпадают с операторами исходного представления группы $P^{X}$.

Введем, по аналогии со случаем $U^{ \pm}$, тотальное множество $\widetilde{M}^{0} \subset \mathrm{INT} H^{0}$ функционалов вида

$$
\Psi_{g}^{0}=2^{-1 / 2} \exp \left(\operatorname{Re} \int_{X} c(z(x)) d m(x)\right) F_{z}^{0}, \quad g \in \operatorname{SL}(2, \mathbb{R})^{X}, \quad \text { где } z=g z_{0} .
$$

ПРЕДЛОЖЕНИЕ 6.7. На множестве $\widetilde{M}^{0}$ скалярное произведение и операторы представления задаются формулами:

$$
\left\langle\Psi_{g_{1}}^{0}, \Psi_{g_{2}}^{0}\right\rangle=\exp \left(\int_{X}\left\langle b^{0}\left(g_{1}(x)\right), b^{0}\left(g_{2}(x)\right)\right\rangle d m(x)\right),
$$


где $b^{0}(g)-1-к о ц и к л P \rightarrow \mathscr{H}^{0}$, заданный равенством

$$
\begin{gathered}
b^{0}(g)=2^{-1 / 2}(b(g), \overline{b(g)}) ; \\
U^{0}\left(g_{1}\right) \Psi_{g}^{0}=\exp \left(-\int_{X} \widetilde{u}\left(g_{1}(x), g(x)\right) d m(x)\right) \Psi_{g_{1} g}^{0},
\end{gathered}
$$

где

$$
\widetilde{u}\left(g_{1}, g\right)=\operatorname{Re} u\left(g_{1}, z\right)=\frac{1}{2}\left\|b\left(g_{1}\right)\right\|^{2}+\left\langle\widetilde{T}^{0}\left(g_{1}\right) b^{0}(g), b^{0}\left(g_{1}\right)\right\rangle .
$$

ДокАЗАТЕЛЬство. Из определения функционалов $\Psi^{0}$ следует, что

$$
\begin{aligned}
\left\langle\Psi_{g_{1}}^{0}, \Psi_{g_{2}}^{0}\right\rangle & =\exp \left(\operatorname{Re} \int_{X}\left\langle b\left(g_{1}(x)\right), b\left(g_{2}(x)\right)\right\rangle d m(x)\right) \\
U^{0}\left(g_{1}\right) \Psi_{g}^{0} & =\exp \left(-\operatorname{Re} \int_{X} u\left(g_{1}(x), g(x)\right) d m(x)\right) \Psi_{g_{1} g}^{0},
\end{aligned}
$$

где $u\left(g_{1}, g\right)$ задается равенством (6.28). Остается заметить, что $\|b(g)\|=\left\|b^{0}(g)\right\|$ и $\operatorname{Re}\left\langle\widetilde{T}^{+}\left(g_{1}\right) b(g), b\left(g_{1}\right)\right\rangle=\left\langle\widetilde{T}^{0}\left(g_{1}\right) b^{0}(g), b^{0}\left(g_{1}\right)\right\rangle$.

По аналогии с теоремами 6.2 и 6.3 получаем следующее утверждение.

Теорема 6.5. Операторь $U^{0}(g)$ сохраняют скалярные произведения $\left\langle\Psi_{g_{1}}^{0}, \Psi_{g_{2}}^{0}\right\rangle$ и продолжаются до ортогональных (не проективных) операторов на всем пространстве INT $H^{0}$.

6.6. Связь с фоковскими представлениями группы $\mathrm{SL}(2, \mathbb{R})^{X}$. $\mathrm{Y}_{\mathrm{c}-}$ тановим связь интегральной модели $U^{+}$представления группы $\operatorname{SL}(2, \mathbb{R})^{X}$ с фоковским представлением этой группы.

По определению, фоковское представление $V^{+}$группы $\operatorname{SL}(2, \mathbb{R})^{X}$, ассоциированное с унитарным представлением $\widetilde{T}^{+}$группы $\mathrm{SL}(2, \mathbb{R})$ в пространстве $\mathscr{H}^{+}$ и 1 -коциклом $b: \operatorname{SL}(2, \mathbb{R}) \rightarrow \mathscr{H}^{+}$, действует в гильбертовом пространстве $\widetilde{H}=$ EXP $\mathscr{H}^{X}$, где

$$
\operatorname{EXP} \mathscr{H}^{X}=\bigoplus_{k=0}^{\infty} S^{k} \mathscr{H}^{X}
$$

и

$$
\mathscr{H}^{X}=\int_{X}^{\oplus} \mathscr{H}_{x}^{+} d m(x), \quad \mathscr{H}_{x}^{+}=\mathscr{H}^{+} .
$$

Рассмотрим тотальное подмножество $\mathscr{M}^{+} \subset \operatorname{EXP} \mathscr{H}^{X}$ векторов вида $\Phi_{g_{1}}^{+}=$ $\operatorname{EXP} b^{X}\left(g_{1}\right), g_{1} \in \mathrm{SL}(2, \mathbb{R})^{X}$, где $b^{X}(g)-1$-коцикл $\mathrm{SL}(2, \mathbb{R})^{X} \rightarrow \mathscr{H}^{X}$, порожденный 1-коциклом $b^{+}: \mathrm{SL}(2, \mathbb{R}) \rightarrow \mathscr{H}^{+}$.

На этом подмножестве скалярные произведения и операторы фоковского представления задаются следующими формулами:

$$
\begin{aligned}
& \left\langle\Phi_{g_{1}}^{+}, \Phi_{g_{2}}^{+}\right\rangle=\exp \left(\int_{X}\left\langle b\left(g_{1}(x)\right), b\left(g_{2}(x)\right)\right\rangle d m(x)\right), \\
& V^{+}(g) \Phi_{g_{1}}^{+}=\exp \left(\int_{X} v\left(g(x), g_{1}(x)\right) d m(x)\right) \Phi_{g g_{1}}^{+},
\end{aligned}
$$

где

$$
v\left(g, g_{1}\right)=-\frac{1}{2}\|b(g)\|^{2}-\left\langle T(g) b\left(g_{1}\right), b(g)\right\rangle \quad \text { для любых } g, g_{1} \in \operatorname{SL}(2, \mathbb{R}) .
$$


Теорема 6.6. Продолжение на $\mathrm{SL}(2, \mathbb{R})^{X}$ интегральной модели $U^{+}$представления группы $P^{X}$ в пространстве INT $H^{+}$проективно эквивалентно фоковской модели $V^{+}$представления группъ $\mathrm{SL}(2, \mathbb{R})^{X}$.

В самом деле, при биекции $\Psi_{g}^{+} \mapsto \Phi_{g}^{+}$тотальных подмножеств $\widetilde{M}^{+}$и $\mathscr{M}^{+}$ в пространствах этих представлений скалярные произведения сохраняются, а формулы для соответствующих операторов $U^{+}(g)$ и $V^{+}(g)$ различаются лишь множителем:

$$
U^{+}(g)=\exp \left(-i \int_{X} c\left(g(x) z_{0}\right) d m(x)\right) V^{+}(g) .
$$

Аналогичное утверждение справедливо для интегральной модели представления $U^{-}$.

Сравним теперь интегральную модель $U^{0}$ представления группы $\operatorname{SL}(2, \mathbb{R})^{X}$ и фоковское представление $V^{0}$ этой группы, ассоциированное с ортогональным представлением $\widetilde{T}^{0}$ группы $\mathrm{SL}(2, \mathbb{R})$ в пространстве $\mathscr{H}^{0}$ и 1-коциклом $b^{0}: \operatorname{SL}(2, \mathbb{R}) \rightarrow \mathscr{H}^{0}$.

В этом случае фоковское представление $V^{0}$ является настоящим (непроективным), и на соответствующих тотальных подмножествах $M^{0}$ и $\mathscr{M}^{0}$ в пространствах представлений скалярные произведения и формулы для операторов представления совпадают. Отсюда вытекает следующая теорема.

Теорема 6.7. Продолжение на $\mathrm{SL}(2, \mathbb{R})^{X}$ интегралъной модели $U^{0}$ ортогонального представления группы $P^{X}$ в пространстве $\widetilde{H}^{0}$ эквивалентно фоковской модели $V^{0}$ представления группь $\mathrm{SL}(2, \mathbb{R})^{X}$. Сплетающий оператор этих представлений порожден отображением ииклических векторов: $\Psi_{e}^{0} \mapsto$ $\Phi_{e}^{0}=\operatorname{EXP} 0$

6.7. Добавление: унитарные представления группы $\widetilde{G}^{X}$, где $\widetilde{G}-$ универсальная накрывающая группы $G=\mathrm{SL}(2, \mathbb{R})$. В этом пункте через $G$ обозначена группа $\mathrm{SL}(2, \mathbb{R})$, а через $\widetilde{G}$ - ее универсальная накрывающая, т. е. накрытие над $G$, где слой над $g \in G$ есть множество $\mathbb{Z}$ классов гомотопически эквивалентных путей на $G$ из единичного элемента $e$ в $g$. Элементы из $\widetilde{G}$ условимся обозначать через $\tilde{g}$, а их образы в $G$ - через $g$.

Поскольку $G$ является факторгруппой группы $\widetilde{G}$, интегральные модели проективных представлений $U^{ \pm}$ее группы токов индуцируют проективные представления $\widetilde{U}^{ \pm}$группы $\widetilde{G}^{X}$ в тех же гильбертовых пространствах INT $H^{ \pm}$. Будет показано, что определенные так проективные представления группы $\widetilde{G}^{X}$ проективно эквивалентны ее унитарным непроективным представлениям $V^{ \pm}$в тех же пространствах $\widetilde{H}^{ \pm}$. Приведем описание этих представлений $V^{ \pm}$, ограничившись для определенности случаем $V^{+}=V$. Для описания представления $V$ достаточно определить действие операторов представления на элементах тотального подмножества функционалов $F_{z}$.

Введем функцию $\psi(\tilde{g}, z)$ на $\widetilde{G} \times L$, где $L$ - верхняя полуплоскость. Положим

$$
\varphi(g, z)=-\log (\beta z+\alpha) \quad \text { при } g=\left(\begin{array}{ll}
\alpha & \beta \\
\gamma & \delta
\end{array}\right) \in G \text { и } z \in L,
$$

где, как и прежде, $\log$ - ветвь логарифма на плоскости с разрезом вдоль вещественной отрицательной полуоси такая, что $\log 1=0$. Функция $\varphi$ всюду 
конечна, и при любом фиксированном $z \in L$ она является однозначной аналитической функцией от $g \in G$ в достаточно малой окрестности единичного элемента $e$. Поэтому для любого $g \in G$ и любого пути $\tilde{g}$ на $G$ из $e$ в $g$ определено ее аналитическое продолжение вдоль этого пути из точки $e$ в точку $g$. Обозначим это аналитическое продолжение через $\psi(\tilde{g}, z)$. Определенная так функция $\psi(\tilde{g}, z)$ зависит только от гомотопического класса пути $\tilde{g}$, а потому является функцией на $\widetilde{G} \times L$.

Из определения следует:

$$
\psi(\tilde{g}, z)=-\log (\beta z+\alpha) \quad \text { при } g=\left(\begin{array}{ll}
\alpha & \beta \\
\gamma & \delta
\end{array}\right),
$$

если $g \in G$ и путь $\tilde{g}$ из $e$ в $g$ принадлежит достаточно малой окрестности единичного элемента $e$.

ПреДЛОжеНИЕ 6.8. При любъх $\tilde{g}_{1}, \tilde{g}_{2} \in \widetilde{G} u z \in L^{+}$имеет место равенство

$$
\psi\left(\tilde{g}_{1} \tilde{g}_{2}, z\right)=\psi\left(\tilde{g}_{1}, g_{2} z\right)+\psi\left(\tilde{g}_{2}, z\right) .
$$

ДокАЗАТЕЛЬСтво. Воспользуемся равенством

$$
\beta z+\alpha=\left(\beta_{1}\left(g_{2} z\right)+\alpha_{1}\right)\left(\beta_{2} z+\alpha_{2}\right),
$$

где $\left(\alpha_{1}, \beta_{1}\right),\left(\alpha_{2}, \beta_{2}\right)$ и $(\alpha, \beta)$ - элементы матриц $g_{1}, g_{2}$ и $g_{1} g_{2}$ соответственно. Из него при $g_{1}, g_{2}$, достаточно близких к единичному элементу, следует:

$$
\log (\beta z+\alpha)=\log \left(\beta_{i}\left(g_{2} z\right)+\alpha_{1}\right)+\log \left(\beta_{2} z+\alpha_{2}\right) .
$$

Таким образом, в силу (6.45), равенство (6.46) справедливо для элементов $g_{1}, g_{2}$ и путей $\tilde{g}_{1}, \tilde{g}_{2}$, достаточно близких к единице. Поэтому оно сохраняется и при аналитическом продолжении по $g$, т. е. остается справедливым для любых $\tilde{g}_{1}, \tilde{g}_{2}$. Предложение 6.8 доказано.

Поставим в соответствие каждой паре $\tilde{g} \in \widetilde{G}^{X}$ и $z \in L^{X}$ следующую функцию на $X$ :

$$
\Psi_{\tilde{g}, z}(x)=\psi(\tilde{g}(x), z(x)) .
$$

Из предложения 6.8 вытекает следующее утверждение.

ПРЕДЛОЖЕНИЕ 6.9. Функиии $\Psi_{\tilde{g}, z}$ связаны соотношением:

$$
\Psi_{\tilde{g}_{1} \tilde{g}_{2}, z}=\Psi_{\tilde{g}_{1}, g_{2} z}+\Psi_{\tilde{g}_{2}, z} .
$$

ОПРедЕлЕниЕ 13. Определим действие операторов $V(\tilde{g}), \tilde{g} \in \widetilde{G}^{X}$, на функции $F_{z}$ тотального множества $M$ следующей формулой:

$$
V(\tilde{g}) F_{z}=\exp \left(\int_{X} \Psi_{\tilde{g}, z}(x) d m(x)\right) F_{g z} .
$$

Tеорема 6.8. Операторы $V(\tilde{g})$ унитарны на $M$, m.e.

$$
\left\langle V(\tilde{g}) F_{z_{1}}, V(\tilde{g}) F_{z_{2}}\right\rangle=\left\langle F_{z_{1}}, F_{z_{2}}\right\rangle \quad \text { для любых } z_{1}, z_{2} \in L^{X} \text { u } \tilde{g} \in \widetilde{G}^{X}
$$


и удовлетворяют соотношению

$$
V\left(\tilde{g}_{1} \tilde{g}_{2}\right) F_{z}=V\left(\tilde{g}_{1}\right) V\left(\tilde{g}_{2}\right) F_{z} \quad \text { для любъх } \tilde{g}_{1}, \tilde{g}_{2} \in \widetilde{G}^{X} u z \in L^{X} .
$$

Тем самым, они порождают унитарное (непроективное) представление группы $\widetilde{G}^{X}$ в пространстве $\widetilde{H}$.

ДокАЗАТЕЛЬство. Групповое свойство (6.51) следует непосредственно из предложения 6.9. Именно:

$$
\begin{aligned}
V\left(\tilde{g}_{1}\right) V\left(\tilde{g}_{2}\right) F_{z} & =\exp \left(\int_{X}\left(\Psi_{\tilde{g}_{1}, g_{2} z}(x)+\Psi_{\tilde{g}_{2}, z}(x)\right) d m(x)\right) F_{g_{1} g_{2} z} \\
& =\exp \left(\int_{X} \Psi_{\tilde{g}_{1} \tilde{g}_{2}, z}(x) d m(x)\right) F_{g_{1} g_{2} z}=U\left(\tilde{g}_{1} \tilde{g}_{2}\right) F_{z} .
\end{aligned}
$$

Далее, ввиду односвязности группы $\widetilde{G}$, свойство унитарности (6.50) достаточно установить только для элементов $\tilde{g} \in \widetilde{G}^{X}$, достаточно близких к единичному элементу. Из определения операторов $V(\tilde{g})$ и формулы (6.23) следует:

$$
\left\langle V(\tilde{g}) F_{z_{1}}, V(\tilde{g}) F_{z_{2}}\right\rangle=\exp \left(\int_{X} u\left(\tilde{g}, z_{1}, z_{2}\right) d m(x)\right),
$$

где

$$
u\left(\tilde{g}, z_{1}, z_{2}\right)=\Psi_{\tilde{g}, z_{1}}+\overline{\Psi_{\tilde{g}, z_{2}}}-c\left(g z_{1}, g z_{2}\right) .
$$

При сделанном предположении имеем, согласно (6.45):

$$
\Psi_{\tilde{g}, z_{1}}+\overline{\Psi_{\tilde{g}, z_{2}}}=-\log \left(\beta z_{1}+\alpha\right)-\log \left(\beta \bar{z}_{2}+\alpha\right) .
$$

С другой стороны, из равенства

$$
g z_{1}-\overline{g z_{2}}=\frac{z_{1}-\bar{z}_{2}}{\left(\beta z_{1}+\alpha\right)\left(\beta \bar{z}_{2}+\alpha\right)}
$$

следует, что

$$
c\left(g z_{1}, g z_{2}\right)=c\left(z_{1}, z_{2}\right)-\log \left(\beta z_{1}+\alpha\right)-\log \left(\beta \bar{z}_{2}+\alpha\right) .
$$

Таким образом, $u\left(\tilde{g}, z_{1}, z_{2}\right)=-c\left(z_{1}, z_{2}\right)$. Отсюда следует (6.50).

Теорема 6.9. Построенное представление $V$ группы $\widetilde{G}^{X}$ проективно эквивалентно представлению $\widetilde{U}$ этой группь.

Утверждение непосредственно следует из формул для операторов этих представлений на тотальном подмножестве $M^{+}$.

\section{7. Интегральные модели представлений группы токов $\mathrm{U}(n, 1)^{X}$}

7.1. Исходные определения. Реализуем $\mathrm{U}(n, 1)$ как группу линейных преобразований в $\mathbb{C}^{n+1}$, сохраняющих эрмитову форму $x_{1} \bar{x}_{n+1}+x_{n+1} \bar{x}_{1}+$ $\left|x_{2}\right|^{2}+\cdots+\left|x_{n}\right|^{2}$, и представим ее элементы в форме блочных матриц

$$
g=\left\|g_{i j}\right\|_{i, j=1,2,3},
$$


где на диагонали стоят квадратные матрицы соответственно порядка $1, n-1$ и 1. В этой реализации максимальная параболическая подгруппа $P$ задается как группа нижних клеточно-треугольных матриц и является полупрямым произведением

$$
P=D<N,
$$

где $N$ - максимальная нильпотентная подгруппа клеточных матриц вида

$$
h=\left(\begin{array}{ccc}
1 & 0 & 0 \\
-z^{*} & e_{n-1} & 0 \\
i t-\frac{z z^{*}}{2} & z & 1
\end{array}\right), \quad t \in \mathbb{R}, \quad z \in \mathbb{C}^{n-1}
$$

(группа Гейзенберга порядка $2 n-1)$, а $D \cong \mathbb{C}^{*} \times U(n-1)$ - подгруппа клеточнодиагональных матриц вида $d=\operatorname{diag}\left(\bar{s}^{-1}, u, s\right), s \in \mathbb{C}^{*}, u \in U(n-1)$.

Представим $D$ в виде прямого произведения $D=\mathbb{R}_{+}^{*} \times D_{0}$, где $D_{0}$ - подгруппа матриц $d=\operatorname{diag}(\varepsilon, u, \varepsilon),|\varepsilon|=1$, и положим

$$
P_{0}=D_{0} \curlywedge N
$$

Таким образом,

$$
P=\mathbb{R}_{+}^{*} \curlywedge P_{0}=\left(\mathbb{R}_{+}^{*} \times D_{0}\right) \curlywedge N .
$$

Элементы из $D_{0}$ и $N$ будем отождествлять соответственно с парами $(\varepsilon, u)$, где $\varepsilon \in U(1), u \in U(n-1)$, и парами $(t, z)$, где $t \in \mathbb{R}, z \in \mathbb{C}^{n-1}$ (вектор-строка). Иногда будем также вместо $(t, z) \in N$ писать $(\zeta, z)$, где $\zeta=i t-|z|^{2} / 2$. В этих обозначениях групповые соотношения имеют вид:

$$
\begin{aligned}
\left(\zeta_{1}, z_{1}\right)\left(\zeta_{2}, z_{2}\right) & =\left(\zeta_{1}+\zeta_{2}-z_{1} z_{2}^{*}, z_{1},+z_{2}\right), \\
(\varepsilon, u)^{-1}(\zeta, z)(\varepsilon, u) & =(\zeta, \bar{\varepsilon} z u), \\
r(\zeta, z) r^{-1} & =\left(r^{2} \zeta, r z\right) \quad \text { при } r \in \mathbb{R}_{+}^{*} .
\end{aligned}
$$

7.2. Описание канонических неприводимых представлений группы $P_{0}$. Имеется, с точностью до сопряженности относительно группы автоморфизмов $\mathbb{R}_{+}^{*}$, два счетных набора $T_{m}^{ \pm}, m=0,1, \ldots$, канонических неприводимых унитарных представлений группы $P_{0}$. Опишем сначала представления $T_{m}^{+}$.

Введем унитарное представление группы $P_{0}$ в гильбертовом пространстве $H=H^{+}$функций $f(z)$ на $\mathbb{C}^{n-1}$ с нормой

$$
\|f\|^{2}=\int_{\mathbb{C}^{n-1}}|f(z)|^{2} \exp \left(-z z^{*}\right) d \mu(z), \quad z z^{*}=\sum z_{i} \bar{z}_{i},
$$

где $d \mu(z)$ - лебегова мера на $\mathbb{C}^{n-1}$, нормированная условием

$$
\int_{\mathbb{C}^{n-1}} \exp \left(-z z^{*}\right) d \mu(z)=1 .
$$

Операторы представления группы $P_{0}$ задаются следующими формулами:

$$
\begin{aligned}
& \left(T^{+}(g) f\right)(z)=\exp \left(\zeta_{0}-z z_{0}^{*}\right) f\left(z+z_{0}\right) \quad \text { при } g=\left(\zeta_{0}, z_{0}\right) \in N, \\
& \left(T^{+}(g) f\right)(z)=f(\bar{\varepsilon} z u) \quad \text { при } g=(\varepsilon, u) \in D_{0} .
\end{aligned}
$$


Соответственно, операторы $T_{r}^{+}$представлений, сопряженных с $T^{+}$, относительно группы автоморфизмов $\mathbb{R}_{+}^{*}$, действуют по формулам:

$$
\begin{aligned}
& \left(T_{r}^{+}(g) f\right)(z)=\exp \left(r^{2} \zeta_{0}-r z z_{0}^{*}\right) f\left(z+r z_{0}\right) \quad \text { при } g=\left(\zeta_{0}, z_{0}\right) \in N, \\
& \left(T_{r}^{+}(g) f\right)(z)=f(\bar{\varepsilon} z u) \quad \text { при } g=(\varepsilon, u) \in D_{0} .
\end{aligned}
$$

Заметим, что мультипликатор $e^{\zeta_{0}-z z_{0}^{*}}$ в формуле для операторов подгруппы $N$ является целой аналитической функцией от $z$. Отсюда следует, что пространство $H^{+}$есть прямая сумма неприводимых попарно не эквивалентных инвариантных подпространств

$$
H^{+}=\bigoplus_{m=1}^{\infty} H_{m}^{+},
$$

где $H_{m}^{+}$- инвариантное подпространство, ииклически порожденное однороднъми полиномами от $\bar{z}_{1}, \ldots, \bar{z}_{n-1}$ степени однородности $m$. В частности, элементами пространства $H_{0}^{+}$являются целые аналитические функиии на $\mathbb{C}^{n-1}$.

Обозначим через $T_{m}^{+}$ограничения операторов $T^{+}$представления группы $P_{0}$ на подпространства $H_{m}^{+}$.

ПреДЛОЖЕНИЕ 7.1. Представления $T_{m}^{+}$группъ $P_{0}$ в пространствах $\mathrm{H}_{m}^{+}$ являются каноническими и обладают единственным почти инвариантным вектором $\varphi_{m}(z)=l_{m}^{n-2}\left(z z^{*}\right)$, где $l_{m}^{n-2}-$ полином Лагерра.

ДокАЗАТЕЛЬство. Найдем все почти инвариантные векторы в $H_{m}^{+}$. Очевидно, что каждый такой вектор $f_{m}(z)$ инвариантен относительно подгруппы $U(n-1)$, а потому является функцией от $z z^{*}, f(z)=\varphi\left(z z^{*}\right)$. Заметим далее, что для любого $m$ прямая сумма пространств $\bigoplus_{k=1}^{m} H_{k}^{+}$содержит все векторы $\left(z z^{*}\right)^{k}$ с $k \leqslant m$, но не содержит векторов $\left(z z^{*}\right)^{k}$ с $k>m$. Отсюда следует, что, с точностью до множителя, $H_{m}$ содержит единственный вектор $f_{m}(z)$ вида $\varphi\left(z z^{*}\right)$, причем этот вектор получается на $m$-м шаге ортогонализации последовательности векторов $1, z z^{*},\left(z z^{*}\right)^{2}, \ldots$ относительно нормы в $H^{+}$. Легко убедиться, что получаемый при такой ортогонализации этот вектор есть, с точностью до множителя, полином Лагерра от $z z^{*}: f_{m}(z)=l_{m}^{n-2}\left(z z^{*}\right)$. Далее, из очевидной оценки: $\left(T_{r}^{+}(g) f_{m}\right)(z)=f_{m}(z)+O(r)$ при $g \in N$ следует, что вектор $f_{m}(z)$ почти инвариантен. Следовательно, согласно предложению 3.2 , представление $T_{m}^{+}$канонично. Предложение 7.1 доказано.

Второе семейство $T_{m}^{-}$канонических неприводимых унитарных представлений группы $P_{0}$ получается из семейства $T_{m}^{+}$операцией комплексного сопряжения, именно, представление $T_{m}^{-}$действует в пространстве функций $\overline{f(z)}$, где $f(z) \in H_{m}^{+}$, операторами вида

$$
T^{-}(g) \bar{f}=\overline{T^{+}(g) f} .
$$

В частности, представление $T_{0}^{-}$действует в пространстве целых антианалитических функций на $\mathbb{C}^{n-1}$.

$\mathrm{C}$ каждой парой $T_{m}^{+}, T_{m}^{-}$неприводимых унитарных представлений группы $P_{0}$ связано неприводимое ортогональное представление $T_{m}^{0}$ группы $P_{0}$ в пространстве $H_{m}^{0} \subset H_{m}^{+} \oplus H_{m}^{-}$векторов вида $(f, \bar{f}), f \in H_{m}^{+}$. Операторы представления 
определяются как ограничение на $H_{m}^{0}$ операторов $T_{m}^{+}(g) \oplus T_{m}^{-}(g)$ на $H_{m}^{+} \oplus H_{m}^{-}$, т. е.

$$
T_{m}^{0}(g)(f, \bar{f})=\left(T_{m}^{+}(g) f, T_{m}^{-}(g) \bar{f}\right)=\left(T_{m}^{+}(g) f, \overline{T_{m}^{+}(g) f}\right) .
$$

C представлениями $T_{m}^{ \pm}$группы $P_{0}$ ассоциированы попарно не эквивалентные неприводимые унитарные представления $\widetilde{T}_{m}^{ \pm}$максимальной параболической группы $P$ в пространствах $\mathscr{H}_{m}^{ \pm}=\int_{0}^{\infty} H_{m, r}^{ \pm} r^{-1} d r, H_{m, r}^{ \pm}=H_{m}^{ \pm}$, и попарно не эквивалентные интегральные модели $U_{m}^{ \pm}=\operatorname{INT} T_{m}^{ \pm}$неприводимых унитарных представлений группы токов $P^{X}$. Представления $\widetilde{T}_{m}^{ \pm}$группы $P$ обладают нетривиальными 1-коциклами $b_{m}^{ \pm}: P \rightarrow \mathscr{H}_{m}^{ \pm}$,

$$
b_{m}^{ \pm}(g)(g)=T^{ \pm}(g) f_{m}(r, z)-f_{m}(r, z), \quad \text { где } f_{m}(r, z)=e^{-r} l_{m}^{n-2}\left(z z^{*}\right),
$$

где $l_{m}^{n-2}-$ полином Лагерра.

Соответственно, с представлениями $T_{m}^{0}$ группы $P_{0}$ ассоциированы попарно не эквивалентные неприводимые ортогональные представления $\widetilde{T}_{m}^{0}$ группы $P$ в пространствах $\mathscr{H}_{m}^{0} \subset \mathscr{H}_{m}^{+} \oplus \mathscr{H}_{m}^{-}$и попарно не эквивалентные интегральные модели $U_{m}^{0}=\mathrm{INT} T_{m}^{0}$ неприводимых ортогональных представлений группы токов $P^{X}$. Представления $\widetilde{T}_{m}^{0}$ группы $P$ обладают нетривиальными 1 -коциклами $b^{0}(g)=\left(b^{+}(g), b^{-}(g)\right)$.

7.3. Представления группы $P$, ассоциированные с представлениями $T^{ \pm}$и $T^{0}$ группы $P_{0}$. В последующих пунктах этого раздела мы ограничиваемся каноническими представлениями подгруппы $P_{0}$ такими, что ассоциированное с ними представление группы $P$ продолжаемо до представления всей группы $\mathrm{U}(n, 1)$. Этим свойством обладают унитарные представления $T_{0}^{ \pm}$, ортогональное представление $T_{0}^{0}$ и только они. Опишем подробно эти представления. Нижний значок 0 в их обозначениях в дальнейшем будем опускать.

Представление $T^{+}$подгруппы $P_{0}$ реализуется в гильбертовом пространстве $H^{+}$целых аналитических функиий $f(z)$ на $\mathbb{C}^{n-1}$ с нормой (7.1); операторы представления задаются на $H$ формулами $(7.2),(7.3)$. Соответственно, операторы сопряженных с $T^{+}$представлений группы $P_{0}$ действуют в пространствах $H_{r}^{+}=H^{+}$по формулам $(7.4),(7.5)$.

Вектором в $H^{+}$, почти инвариантным относительно семейства сопряженных представлений $T_{r}^{+}$группы $P_{0}$, является функция $f(z) \equiv 1$, которую мы будем дальше обозначать через $\mathbb{I}$. Имеем для нее

$$
\left\|T_{r}^{+}(g) \mathbb{I}-\mathbb{I}\right\|<c(g) r \quad \text { для любого } g \in P_{0} .
$$

Ассоциированное с $T^{+}$представление $\widetilde{T}^{+}$максимальной параболической группы $P$ реализуется в прямом интеграле по мере $d^{*} r=r^{-1} d r$ на $\mathbb{R}^{+}$гильбертовых пространств $H_{r}^{+}$,

$$
\mathscr{H}^{+}=\int_{0}^{\infty} H_{r}^{+} d^{*} r,
$$

т. е. элементами $\mathscr{H}^{+}$являются сечения $f(r)$ расслоения над $\mathbb{R}_{+}^{*}$, где слоем над $r \in \mathbb{R}_{+}^{*}$ является пространство $H_{r}^{+}$, удовлетворяющие условию

$$
\int_{0}^{\infty}\|f(r)\|^{2} r^{-1} d r<\infty .
$$


В этой реализации операторы подгруппы $P_{0}$ действуют на каждом пространстве $H_{r}^{+}$по формулам $(7.4),(7.5)$, а операторы гомотетии $T\left(r_{0}\right), r_{0} \in \mathbb{R}_{+}^{*}$, изометрически отображают $H_{r}$ в $H_{r_{0} r}$.

Таким образом, представление $\widetilde{T}^{+}$группы $P$ действует в пространстве $\mathscr{H}^{+}$ по формулам:

$$
\begin{aligned}
& \left(\widetilde{T}^{+}(g) f\right)(r, z)=\exp \left(r^{2}\left(i t_{0}-\frac{\left|z_{0}\right|^{2}}{2}\right)-r z z_{0}^{*}\right) f\left(r, z+r z_{0}\right) \text { при } g=\left(t_{0}, z_{0}\right) \in N, \\
& \left(\widetilde{T}^{+}(g) f\right)(r, z)=f(r, \bar{\varepsilon} z u) \quad \text { при } g=(\varepsilon, u) \in D_{0}, \\
& \left(\widetilde{T}^{+}(g) f\right)(r, z)=f\left(r_{0} r, z\right) \quad \text { при } g=r_{0} \in \mathbb{R}_{+}^{*} .
\end{aligned}
$$

Представление $\widetilde{T}^{+}$группы $P$ имеет нетривиальный 1 -коцикл $b^{+}: P \rightarrow \mathscr{H}^{+}$:

$$
b^{+}(g)=\widetilde{T}^{+}(g) f_{0}(r, z)-f_{0}(r, z), \quad \text { где } f_{0}(r, z)=e^{-r^{2}} .
$$

Второе каноническое унитарное представление $T^{-}$подгруппы $P_{0}$ и ассоциированное с ним унитарное представление группы $P$ действуют соответственно в пространствах $H^{-}$и $\mathscr{H}^{-}=\int_{0}^{\infty} H_{r}^{-} d^{*} r$, где $H^{-}-$пространство цельх антианалитических функций $f(z)$ на $\mathbb{C}^{n-1}$ с нормой (7.1).

Наконец, каноническое ортогональное представление $T^{0}$ подгруппы $P_{0}$ и ассоциированное с ним ортогональное представление группы $P$ действуют соответственно в пространствах $H^{0}$ и $\mathscr{H}^{0}=\int_{0}^{\infty} H_{r}^{-} r^{-1} d r$, где $H^{0} \subset H^{+} \oplus H^{-}-$ подпространство векторов $(f, \bar{f}), f \in H^{+}$. Операторы $T^{0}(g)$ на $H^{0}$ получаются ограничением на $H^{0}$ операторов $T^{+}(g) \oplus T^{-}(g)$ на $H^{+} \oplus H^{-}$, где $H^{-}-$ пространство целых антианалитических функций $f(z)$ на $\mathbb{C}^{n-1}$ с нормой (7.1).

7.4. Представления группы $P^{X}$, ассоциированные с представлениями $T^{ \pm}$и $T^{0}$ группы $P_{0}$. В соответствии с общими определениями, унитарные представления $U^{ \pm}$группы $P^{X}$, ассоциированные с представлениями $T^{ \pm}$ группы $P_{0}$, реализуются в прямых интегралах

$$
\operatorname{INT} H^{ \pm}=\int_{l_{+}^{1}(X)}^{\oplus} H_{\xi}^{ \pm} d \mathscr{L}(\xi),
$$

где $H_{\xi}^{ \pm}, \xi=\left\{r_{k}, x_{k}\right\},-$ счетные тензорные степени гильбертова пространства $H^{ \pm}$со стабилизирующим вектором $\mathbb{I}$ :

$$
H_{\xi}^{ \pm}=\bigotimes_{k=1}^{\infty} H_{r_{k}}^{ \pm}, \quad H_{r_{k}}^{ \pm}=H^{ \pm} .
$$

Таким образом, элементами INT $H^{ \pm}$являются сечения $F(\tilde{r})$ расслоения над $D_{+}$ со слоем $H_{\tilde{r}}^{ \pm}$.

Операторы $U^{ \pm}(g), g \in P_{0}^{X}$, действуют в слоях $\mathscr{H}_{\tilde{r}}^{ \pm}$расслоения по формуле

$$
U^{ \pm}(g(\cdot))=\bigotimes_{k=1}^{\infty} T_{r_{k}}^{ \pm}\left(g\left(x_{k}\right)\right) .
$$


Операторы $U^{ \pm}\left(r_{0}(\cdot)\right), r_{0} \in \mathbb{R}^{X}$, задаются формулой

$$
\left(U^{ \pm}\left(r_{0}(\cdot)\right) f\right)(\xi)=\exp \left(\frac{1}{2} \int_{X} \log r_{0}(x) d m(x)\right) f\left(r_{0}(\cdot) \xi\right) .
$$

Их групповое свойство и унитарность операторов $U^{ \pm}(g)$ при $g \in P_{0}^{X}$ очевидны, а унитарность операторов $U^{ \pm}\left(r_{0}(\cdot)\right), r_{0} \in \mathbb{R}^{X}$, следует из проективной инвариантности меры $\mathscr{L}$.

Аналогично, ортогональное представление группы $P^{X}$, ассоциированное с представлением $T^{0}$ группы $P_{0}$, реализуется в прямом интеграле

$$
\operatorname{INT} H^{0}=\int_{l_{+}^{1}(X)}^{\oplus} H_{\xi}^{0} d \mathscr{L}(\xi),
$$

где $H_{\xi}^{0}, \xi=\left\{r_{k}, x_{k}\right\},-$ счетные тензорные степени вещественного гильбертова пространства $H^{0} \subset H^{+} \oplus H^{-}$со стабилизирующим вектором $2^{-1 / 2}(\mathbb{I}, \mathbb{I})$, а операторы $U^{0}\left(r_{0}(\cdot)\right), r_{0} \in \mathbb{R}^{X}$, задаются формулой, аналогичной (7.10).

7.5. Продолжение представлений $\widetilde{T}^{ \pm}$и $\widetilde{T}^{0}$ подгруппы $P$ до представлений группы $\mathrm{U}(n, 1)$. Конструкция этих продолжений аналогична конструкции продолжений для случая $\mathrm{SL}(2, \mathbb{R})$, приведенной в предыдущем разделе. Опишем сначала продолжения на $\mathrm{U}(n, 1)$ представления $\widetilde{T}^{+}$подгруппы $P$.

Введем, по аналогии с плоскостью Лобачевского для случая $\operatorname{SL}(2, \mathbb{R})$, однородное пространство $L=\mathrm{U}(n, 1) / K$, где $K$ - максимальная компактная подгруппа ( $n$-мерное комплексное пространство Лобачевского). В принятой матричной реализации $K$ есть подгруппа в $\mathrm{U}(n, 1)$ клеточных матриц вида

$$
\begin{gathered}
\left(\begin{array}{ccc}
\lambda & a & \mu \\
b & c & b \\
\mu & a & \lambda
\end{array}\right), \\
L=\left\{v=(a, b) \in \mathbb{C} \oplus \mathbb{C}^{n-1} \mid a+\bar{a}+b^{*} b<0\right\}, \quad \text { где } b^{*} b=\sum \bar{b}_{i} b_{i}
\end{gathered}
$$

( $b$ - вектор-столбец), и действие $v \mapsto g v$ на $L$ элементов $g=\left\|g_{i j}\right\|_{i, j=1,2,3}$ группы $\mathrm{U}(n, 1)$ задается так: $g(a, b)=\left(a^{\prime}, b^{\prime}\right)$, где

$$
\begin{aligned}
& a^{\prime}=\left(g_{11}+g_{12} b+g_{13} a\right)^{-1}\left(g_{31}+g_{32} b+g_{33} a\right), \\
& b^{\prime}=\left(g_{11}+g_{12} b+g_{13} a\right)^{-1}\left(g_{21}+g_{22} b+g_{23} a\right) .
\end{aligned}
$$

В частности,

$$
\begin{aligned}
& g(a, b)=\left(a+\zeta_{0}+\left(z_{0}, b\right), b-z_{0}^{*}\right) \quad \text { при } g=\left(\zeta_{0}, z_{0}\right) \in N, \\
& g(a, b)=(a, u b \bar{\varepsilon}) \quad \text { при } g=(\varepsilon, u) \in D_{0}, \\
& g(a, b)=\left(r^{2} a, r b\right) \quad \text { при } g=r \in \mathbb{R}_{+}^{*}, \\
& s(a, b)=\left(a^{-1}, a^{-1} b\right) \quad \text { при инволюции } s=\left(\begin{array}{ccc}
0 & 0 & 1 \\
0 & e_{n-1} & 0 \\
1 & 0 & 0
\end{array}\right) .
\end{aligned}
$$

Неподвижной точкой на $L$ относительно действия подгруппы $K$ является точка $v_{0}=(-1,0)$. 
Введем следующую функцию на $L \times L$ :

$$
p\left(v_{1}, v_{2}\right)=-\left(a_{1}+\bar{a}_{2}+b_{2}^{*} b_{1}\right) \quad \text { при } v_{i}=\left(a_{i}, b_{i}\right) \in L .
$$

Очевидно, что $p\left(v_{0}, v_{0}\right)=2$ при $v_{0}=(-1,0)$, и легко убедиться, что

$$
\operatorname{Re} p\left(v_{1}, v_{2}\right)>0 \quad \text { для любых } v_{1}, v_{2} \in L \text {. }
$$

ПРЕДЛОЖЕНИЕ 7.2. Функция $p\left(v_{1}, v_{2}\right)$, где $v_{1}=\left(a_{1}, b_{1}\right), v_{2}=\left(a_{2}, b_{2}\right) \in L$ u $g \in \mathrm{U}(n, 1)$, удовлетворяет соотношению

$$
p\left(g v_{1}, g v_{2}\right)=p\left(v_{1}, v_{2}\right)\left(g_{11}+g_{12} b_{1}+g_{13} a_{1}\right)^{-1}\left[\overline{\left(g_{11}+g_{12} b_{2}+g_{13} a_{2}\right)}\right]^{-1} .
$$

В частности, функиия $p\left(v_{1}, v_{2}\right)$ инвариантна относительно действия подгруппы $P_{0}$ и $p\left(g v_{1}, g v_{2}\right)=r^{2} p\left(v_{1}, v_{2}\right)$ nрu $g=r \in \mathbb{R}_{+}^{*}$.

ДокАЗАТЕЛЬСтво. Справедливость приведенных соотношений для элементов $g \in P_{0}, r \in \mathbb{R}_{+}^{*}$ и инволюции $s$ следует непосредственно из (7.11). Поскольку эти элементы алгебраически порождают $\mathrm{U}(n, 1)$, отсюда следует их справедливость и для любых элементов $g \in \mathrm{U}(n, 1)$.

Поставим в соответствие каждому $v=(a, b) \in L$ следующую функцию на $\mathbb{R}_{+}^{*} \oplus \mathbb{C}^{n-1}$ :

$$
f_{v}(r, z)=\exp \left(r^{2} a+r(z, b)\right), \quad \text { где }(z, b)=\sum z_{i} b_{i}
$$

Заметим, что в этих обозначениях выражение для 1-коцикла на $\mathscr{H}^{+}$имеет следующий вид:

$$
b^{+}(g)=\widetilde{T}^{+}(g) f_{v_{0}}-f_{v_{0}}, \quad \text { где } v_{0}=(-1,0) .
$$

ПРеДЛОЖЕНИЕ 7.3. При любых фиксированных $v \in L$ u $r$ функиия $f_{v}(r, z)$ принадлежит пространству $H^{+}$, и скалярное произведение в $H^{+}$двух таких функций имеет вид

$$
\left\langle f_{v_{1}}, f_{v_{2}}\right\rangle_{H^{+}}=\exp \left(-r^{2} p\left(v_{1}, v_{2}\right)\right) \quad \text { npu } v_{i}=\left(a_{i}, b_{i}\right)
$$

где $p\left(v_{1}, v_{2}\right)$ задается равенством (7.12). В частности,

$$
\left\|f_{v}\right\|_{H^{+}}^{2}=\exp \left(-r^{2} p(v, v)\right)<\infty .
$$

ДоказАтельство. Имеем:

$$
\left\langle f_{v_{1}}, f_{v_{2}}\right\rangle_{H^{+}}=\int_{\mathbb{C}^{n-1}} \exp \left(r^{2}\left(a_{1}+\bar{a}_{2}\right)+r\left(z, b_{1}\right)+r \overline{\left(z, b_{2}\right)}-z z^{*}\right) d \mu(z) .
$$

Для доказательства (7.17) достаточно воспользоваться следующим равенством:

$$
\int_{\mathbb{C}^{n-1}} \exp \left(r\left(z, b_{1}\right)+r \overline{\left(z, b_{2}\right)}-z z^{*}\right) d \mu(z)=\exp \left(r^{2} b_{2}^{*} b_{1}\right) .
$$


ПРЕДЛОЖЕНИЕ 7.4. Для любых $v_{1}, v_{2} \in L^{X}$ бункиия $f_{v_{1}}-f_{v_{2}}$ принадлежит $\mathscr{H}^{+}$, и скалярное произведение функций $f_{v_{1}}-f_{v_{2}} u f_{v_{1}^{\prime}}-f_{v_{2}^{\prime}}$ в пространстве $\mathscr{H}$ имеет вид:

$$
\left\langle f_{v_{1}}-f_{v_{2}}, f_{v_{1}^{\prime}}-f_{v_{2}^{\prime}}\right\rangle=\frac{1}{2} \sum_{i, j=1,2}(-1)^{i+j-1} \log p\left(v_{i}, v_{j}^{\prime}\right) .
$$

В частности,

$$
\left\|f_{v_{1}}-f_{v_{2}}\right\|^{2}=\frac{1}{2} \log \frac{\left|p\left(v_{1}, v_{2}\right)\right|^{2}}{p\left(v_{1}, v_{1}\right) p\left(v_{2}, v_{2}\right)}<\infty .
$$

Здесь через $\log$, как и всюду ранее, обозначена ветвь логарифма на плоскости $\mathbb{C}$ с разрезом вдоль вещественной отрицательной полуоси, заданная условием $\log 1=0$.

ДокАЗАТЕЛЬСТво. По определению,

$$
\left\langle f_{v_{1}}-f_{v_{2}}, f_{v_{1}^{\prime}}-f_{v_{2}^{\prime}}\right\rangle=\int_{0}^{\infty}\left\langle f_{v_{1}}-f_{v_{2}}, f_{v_{1}^{\prime}}-f_{v_{2}^{\prime}}\right\rangle_{H_{r}^{*}} r^{-1} d r .
$$

Поэтому равенство (7.19) следует непосредственно из (7.15) и соотношения

$$
\int_{0}^{\infty}\left(e^{-a r^{2}}-e^{-b r^{2}}\right) r^{-1} d r=\frac{1}{2}(\log b-\log a) \quad \text { при } \operatorname{Re} a, \operatorname{Re} b>0 .
$$

ОПРЕДЕЛЕНИЕ 14. Обозначим через $M^{+}$множество функций $f_{v_{1}}-f_{v_{2}}$, $v_{1}, v_{2} \in \mathscr{H}^{+}$, в пространстве $\mathscr{H}^{+}$.

Очевидно, что $M^{+}$тотально в $\mathscr{H}^{+}$.

Легко убедиться, что множество $M^{+}$функций $f_{v_{1}}-f_{v_{2}}$ тотально в $\mathscr{H}^{+}$.

ПреДЛОЖенИЕ 7.5. Множество функиий $f_{v}, v \in L$, а значит, и множество $M^{+}$, инвариантны относительно действия операторов представления группы $P$, а именно,

$$
\widetilde{T}^{+}(g) f_{v}=f_{g v} \quad \text { для любих } v \in L u g \in P,
$$

где $g v=g(a, b)$ определяется при $g \in P$ формулами (7.11).

В этом легко непосредственно убедиться, сравнивая между собой выражения для $\widetilde{T}^{+}(g) f_{v}$ и $f_{g v}$.

Таким образом, операторы группы $P$ действуют на множестве $M^{+}$по формуле:

$$
\widetilde{T}^{+}(g)\left(f_{v_{1}}-f_{v_{2}}\right)=f_{g v_{1}}-f_{g v_{2}} \quad \text { для любых } v_{1}, v_{2} \in L .
$$

ОПРЕдЕлЕНиЕ 15. Определим действие операторов $\widetilde{T}^{+}$всей группы $\mathrm{U}(n, 1)$ на множестве $M^{+}$той же формулой (7.20).

Теорема 7.1. Оператори $\widetilde{T}^{+}$сохраняют скалярное произведение на $\mathrm{M}^{+}$, удовлетворяют групповому соотношению и тем самым задают продолжение исходного представления $\widetilde{T}^{+}$подгруппь $Р$ до унитарного представления группь $\mathrm{U}(n, 1)$. 
ДоказАТЕльство. Справедливость группового соотношения очевидна. Инвариантность скалярного произведения следует из явной формулы (7.18) для скалярного произведения и соотношения (7.14) для функции $p\left(v_{1}, v_{2}\right)$.

Определенное так представление $\widetilde{T}^{+}$группы $\mathrm{U}(n, 1)$ является особым, и нетривиальный 1-коцикл $b^{+}: \mathrm{U}(n, 1) \rightarrow \mathscr{H}^{+}$является продолжением на $\mathrm{U}(n, 1)$ 1-коцикла группы $P$, т. е. задается в принятых обозначениях, как и для подгруппы $P$, формулой $b^{+}(g)=T(g) f_{v_{0}}-f_{v_{0}}$.

Аналогично определяется продолжение представления $\widetilde{T}^{-}$группы $P$ до унитарного представления группы $\mathrm{U}(n, 1)$. Именно, множество $M^{+}$функций $f_{v_{1}}-f_{v_{2}}$ заменяется на множество $M^{-}$функций $\overline{f_{v_{1}}}-\overline{f_{v_{2}}}$. Очевидно, что $M^{-}$ тотально в $\mathscr{H}^{-}$и инвариантно относительно действия операторов $\widetilde{T}^{-}$группы $P$ :

$$
\widetilde{T}^{-}(g)\left(\overline{f_{z_{1}}}-\overline{f_{z_{2}}}\right)=\overline{f_{g z_{1}}}-\overline{f_{g z_{2}}} .
$$

Той же формулой (7.21) определяется и продолжение представления $\widetilde{T}^{-}$подгруппы $P$ на всю группу $\mathrm{U}(n, 1)$.

Наконец, определенные так продолжения представлений $\widetilde{T}^{ \pm}$подгруппы $P$ до унитарных представлений группы $\mathrm{U}(n, 1)$ индуцируют продолжение ортогонального представления подгруппы $P$ в пространстве $\mathscr{H}^{0} \subset \mathscr{H}^{+} \oplus \mathscr{H}^{-}$до ортогонального представления группы $\mathrm{U}(n, 1)$.

Приведем в заключение несколько соотношений, которые будут использованы в дальнейшем.

Обозначим:

$$
c\left(v_{1}, v_{2}\right)=\log \left(p\left(v_{1}, v_{2}\right)\right), \quad v_{1}, v_{2} \in L
$$

В этих обозначениях условие инвариантности скалярного произведения на $\mathrm{M}^{+}$ можно представить в виде

$$
\begin{gathered}
c\left(g v_{1}, g v_{0}\right)+\overline{c\left(g v_{2}, g v_{0}\right)}-c\left(g v_{1}, g v_{2}\right)-c\left(g v_{0}, g v_{0}\right) \\
=c\left(v_{1}, v_{0}\right)+\overline{c\left(v_{2}, v_{0}\right)}-c\left(v_{1}, v_{2}\right)-c\left(v_{0}, v_{0}\right)
\end{gathered}
$$

для любого $g \in \mathrm{U}(n, 1)$, где $v_{0}=(-1,0)$. Далее, на основании предложения 7.4 имеем:

$$
\begin{aligned}
& \|b(g)\|^{2}=\frac{1}{2}\left(2 \operatorname{Re} c\left(g v_{0}, v_{0}\right)-c\left(g v_{0}, g v_{0}\right)-\log 2\right), \quad \text { где } v_{0}=(-1,0), \\
& \left\langle T(g) b\left(g_{1}\right), b(g)\right\rangle=\frac{1}{2}\left(c\left(g g_{1} v_{0}, v_{0}\right)+c\left(g v_{0}, g v_{0}\right)-c\left(g g_{1} v_{0}, g v_{0}\right)-c\left(g v_{0}, v_{0}\right)\right) .
\end{aligned}
$$

Из (7.24) и (7.25) также следует:

$$
\begin{aligned}
& -\frac{1}{2}\|b(g)\|^{2}-\left\langle T(g) b\left(g_{1}\right), b(g)\right\rangle=\frac{i}{2} \operatorname{Im} c\left(g z_{0}, v_{0}\right) \\
& \quad+\frac{1}{2}\left(c\left(g g_{1} v_{0}, g v_{0}\right)-c\left(g g_{1} v_{0}, v_{0}\right)\right)-\frac{1}{4}\left(c\left(g v_{0}, g v_{0}\right)-c\left(v_{0}, v_{0}\right)\right) .
\end{aligned}
$$


7.6. Продолжение унитарных представлений $U^{ \pm}$группы токов $P^{X}$ до проективных унитарных представлений группы $\mathrm{U}(n, 1)^{X}$. Конструкция этих продолжений аналогична случаю $\mathrm{SL}(2, \mathbb{R})$. Опишем продолжение на $\mathrm{U}(n, 1)^{X}$ представления $U^{+}$группы $P^{X}$.

Обозначим через $L^{X}$ пространство ограниченных функций $v: X \rightarrow L, v(x)=$ $(a(x), b(x))$. Действие группы $\mathrm{U}(n, 1)$ на $L$ индуцирует поточечное действие на $L^{X}$ группы токов $\mathrm{U}(n, 1)^{X}$ и, в частности, подгруппы $P^{X}$.

Поставим в соответствие каждой функции $v(x)=(a(x), b(x)) \in L^{X}$ функционал $F^{+}$на $l_{+}^{1}(X)$. Именно, свяжем с каждой функцией $v(x)=(a(x), b(x)) \in L^{X}$ и каждой парой $(r, x) \in \mathbb{R}_{+}^{*} \times X$ целую функцию на $\mathbb{C}^{n-1}$ :

$$
f_{v, r, x}(z)=\exp \left(r^{2} a(x)+r(z, b(x))\right)
$$

и определим функционалы $F_{v}^{+}(\xi)=F_{v}^{+}\left(\left\{r_{k}, x_{k}\right\}\right)$ на $l_{+}^{1}(X)$ следующим равенством:

$$
F_{v}^{+}(\xi)=\bigotimes_{k=1}^{\infty} f_{v, r_{k}, x_{k}} \quad \text { при } \xi=\left\{r_{k}, x_{k}\right\} .
$$

ПРеДЛОЖеНИЕ 7.6. Для каждого $v \in L^{X}$ и каждого $\xi \in l_{+}^{1}(X)$ бесконечное тензорное произведение (7.28) сходится и принадлежит пространству $H_{\xi}^{+}$. При этом для любых $v_{1}, v_{2} \in L^{X}$ имеем:

$$
\left\langle F_{v_{1}}^{+}(\xi), F_{v_{2}}^{+}(\xi)\right\rangle_{H_{\xi}^{+}}=\exp \left(-\sum r_{k}^{2} p\left(v_{1}\left(x_{k}\right), v_{2}\left(x_{k}\right)\right)\right),
$$

где функиия $p\left(v_{1}, v_{2}\right)$ задается равенством (7.12).

ДокАЗАтЕЛьство. Заметим, прежде всего, что функции $f_{v, r_{k}, x_{k}}(z)$ принадлежат пространствам $H_{r_{k}}^{+}=H^{+}$, и для любых $v_{1}, v_{2} \in L^{X}$ имеем на основании (7.15):

$$
\left\langle f_{v_{1}, r_{k}, x_{k}}, f_{v_{2}, r_{k}, x_{k}}\right\rangle_{H^{+}}=\exp \left(-r^{2} p\left(v_{1}\left(x_{k}\right), v_{2}\left(x_{k}\right)\right)\right) .
$$

В частности, $\left\|f_{v}\right\|_{H^{+}}^{2}=\exp \left(-r^{2} p(v, v)\right)<\infty$.

Далее, из предложения 7.1 следует, что функции $f_{v, r_{k}, x_{k}}$ удовлетворяют оценке

$$
\left\|f_{v, r_{k}, x_{k}}-\mathbb{I}\right\|<c r_{k} .
$$

Из этой оценки следует, что бесконечное тензорное произведение $F_{v}^{+}(\xi)$ сходится и принадлежит пространству $H_{\xi}^{+}$. Формула (7.29) следует непосредственно из (7.30).

Отметим, что формула (7.29) может быть записана в виде

$$
\left\langle F_{v_{1}}^{+}(\xi), F_{v_{2}}^{+}(\xi)\right\rangle_{H_{\tilde{r}}^{+}}=\exp \left(-\left\langle\tilde{r}^{2}, p\left(v_{1}, v_{2}\right)\right\rangle\right) .
$$

Теорема 7.2. Для каждого $v \in L^{X}$ функционал $F_{v}^{+}(\xi)$ на $l_{+}^{1}(X)$ со значениями в $H_{\xi}^{+}$принадлежит пространству INT $H^{+}, u$ для любых $v_{1}, v_{2} \in L^{X}$ имеем:

$$
\left\langle F_{v_{1}}^{+}, F_{v_{2}}^{+}\right\rangle=c \exp \left(-\frac{1}{2} \int_{X} \log p\left(v_{1}(x), v_{2}(x)\right) d m(x)\right), \quad c=e^{\gamma / 2}
$$

$(\gamma-$ постоянная Эйлера). 
В самом деле, равенство (7.32) следует непосредственно из (7.31) и соотношения (2.9) для меры $\mathscr{L}$, согласно которому

$$
\int_{l_{+}^{1}(X)} \exp \left(-\sum r_{k}^{2} a\left(x_{k}\right)\right) d \mathscr{L}(\xi)=e^{\gamma / 2} \exp \left(-\frac{1}{2} \int_{X} \log a(x) d m(x)\right) .
$$

Сходимость интеграла в правой части равенства (7.32) следует из условия ограниченности функции $v \in L^{X}$.

Обозначим через $M^{+}$определенное таким образом подмножество функционалов $F_{v} \in \mathrm{INT} H^{+}, v \in L^{X}$. Нетрудно убедиться, что это подмножество тотально в INT $H^{+}$.

ТЕОРема 7.3. Множество $M^{+}$инвариантно относительно операторов подгруппи $P_{0}^{X}$, а именно, имеем для любого $v=(a, b) \in L^{X}: U^{+}(g) F_{v}^{+}=F_{g v}^{+}$, где $g v=\left(a+\zeta_{0}+\left(z_{0}, b\right), b-z_{0}^{*}\right)$ npu $g=\left(\zeta_{0}, z_{0}\right) \in N^{X}$ u gv $=(a, u b \bar{\varepsilon}) \quad n p u$ $g=(\varepsilon, u) \in D_{0}^{X}$.

При $g=r_{0} \in\left(\mathbb{R}_{+}^{*}\right)^{X}$ имеем:

$$
U^{+}\left(r_{0}\right) F_{v}^{+}=\exp \left(\int_{X} \log r_{0}(x) d m(x)\right) F_{g v}^{+}, \quad \text { əде } g v=\left(r_{0}^{2} a, r_{0} b\right) .
$$

Утверждения следуют непосредственно из формул для операторов представления и определения функционалов $F_{v}^{+}$.

ОПРЕДЕЛЕНИЕ 16. Определим действие операторов $U^{+}(g)$ группы $\mathrm{U}(n, 1)^{X}$ на множестве $M^{+}$следующим равенством:

$$
U^{+}(g) F_{v}^{+}=\exp \left(\frac{1}{2} \int_{X} \varphi(g(x), v(x)) d m(x)\right) F_{g v}^{+},
$$

где

$$
\varphi(g, v)=\left(c\left(g v, g v_{0}\right)-c\left(v, v_{0}\right)\right)-\frac{1}{2}\left(c\left(g v_{0}, g v_{0}\right)-c\left(v_{0}, v_{0}\right)\right) .
$$

Заметим, что это определение аналогично соответствующему определению функционалов $F_{z}^{+}$для случая группы $\operatorname{SL}(2, \mathbb{R})^{X}$ (см. формулы $(6.24)$ и (6.25)). При этом функция $c\left(v_{1}, v_{2}\right) / 2$ удовлетворяет тем же соотношениям, что и функция $c\left(z_{1}, z_{2}\right)$ в случае $\operatorname{SL}(2, \mathbb{R})$. Поэтому основанные на этом определении утверждения и конструкции для случая $\mathrm{SL}(2, \mathbb{R})$ переносятся и на случай $\mathrm{U}(n, 1)$. Изложим их более кратко.

Прежде всего, ограничения операторов $U^{+}(g)$ на подгруппу $P^{X}$ совпадают с операторами исходного представления группы $P^{X}$.

Далее, подобно случаю $\mathrm{SL}(2, \mathbb{R})$, мы заменяем множество функционалов $F_{v}^{+}$ множеством $\widetilde{M}^{+}$функционалов вида

$$
\Psi_{g}^{+}=(2 c)^{-1 / 2} \exp \left(\frac{1}{2} \int_{X} c\left(v(x), v_{0}\right) d m(x)\right) F_{z}, \quad g \in \mathrm{U}(n, 1)^{X},
$$

где $v=g v_{0}, c=e^{\gamma / 2}$.

На этом множестве скалярное произведение и операторы представления задаются формулами:

$$
\left\langle\Psi_{g_{1}}^{+}, \Psi_{g_{2}}^{+}\right\rangle=\exp \left(\int_{X}\left\langle b^{+}\left(g_{1}(x)\right), b^{+}\left(g_{2}(x)\right)\right\rangle d m(x)\right)
$$


где $b^{+}(g)-1$-коцикл $G \rightarrow \mathscr{H}^{+}$, заданный равенством (7.16);

$$
U^{+}\left(g_{1}\right) \Psi_{g}^{+}=\exp \left(-\int_{X} u\left(g_{1}(x), g(x)\right) d m(x)\right) \Psi_{g_{1} g}^{+},
$$

где

$$
u\left(g_{1}, g\right)=\frac{i}{2} \operatorname{Im} c\left(g_{1} v_{0}, v_{0}\right)+\frac{1}{2}\left\|b\left(g_{1}\right)\right\|^{2}+\left\langle\widetilde{T}\left(g_{1}\right) b(g), b\left(g_{1}\right)\right\rangle .
$$

Отсюда, подобно случаю $\mathrm{SL}(2, \mathbb{R})$, вытекает следующая теорема.

Теорема 7.4. Операторь $U^{+}(g)$ сохраняют скалярные произведения $\left\langle\Psi_{g_{1}}^{+}, \Psi_{g_{2}}^{+}\right\rangle$, и их продолжения на все пространство INT $H^{+}$образуют проективное унитарное представление группь $\mathrm{U}(n, 1)^{X}$.

Продолжение второго представления $U^{-}$группы $P^{X}$ до представления группы $\mathrm{U}(n, 1)^{X}$ получается заменой тотального множества $\widetilde{M}^{+} \subset \mathrm{INT} H^{+}$тотальным множеством $\widetilde{M}^{-} \subset$ INT $H^{-}$функционалов $\Psi_{g}^{-}=\overline{\Psi_{g}^{+}}$. Очевидно, что скалярные произведения $\left\langle\Psi_{g_{1}}^{+}, \Psi_{g_{2}}^{+}\right\rangle$и $\left\langle\Psi_{g_{1}}^{-}, \Psi_{g_{2}}^{-}\right\rangle$комплексно сопряжены. Действие операторов $U^{-}(g)$ группы $\mathrm{U}(n, 1)^{X}$ на INT $M^{-}$задается равенством

$$
U^{-}(g) \Psi_{g}^{-}=\overline{U^{+}(g) \Psi_{g}^{+}} .
$$

Подобно случаю $U^{+}$, ограничения операторов $U^{-}(g)$ на подгруппу $P^{X}$ совпадают с операторами исходного представления подгруппы $P^{X}$ в пространстве INT $H^{+}$, и для них также справедлива теорема 7.4.

7.7. Продолжение ортогонального представления $U^{0}$ группы токов $P^{X}$ до ортогонального представления группы $\mathrm{U}(n, 1)^{X}$. Свяжем с каждой парой $v(x)=(a(x), b(x)) \in L^{X}$ и $(r, x) \in \mathbb{R}_{+}^{*} \times X$ вектор $f_{v, r, x}^{0} \in H_{r}^{0}$,

$$
f_{v, r, x}^{0}=2^{-1 / 2}\left(e^{i r^{2} z(x)}, e^{-i r^{2} \overline{z(x)}}\right),
$$

и определим функционалы $F_{v}^{0}(\xi)$ на $l_{+}^{1}(X)$ следующим равенством:

$$
F_{v}^{0}(\xi)=\bigotimes_{k=1}^{\infty} f_{v, r_{k}, x_{k}}^{0} \text { при } \xi=\left\{r_{k}, x_{k}\right\}
$$

Подобно случаю $\mathrm{SL}(2, \mathbb{R})$, бесконечное тензорное произведение $\bigotimes_{k=1}^{\infty} f_{v, r_{k}, x_{k}}^{0}$ сходится и $F_{v}^{0}(\xi) \in H_{\xi}^{0}$ при любых $v \in L^{X}$ и $\xi \in l_{+}^{1}(X)$. Определенные так функционалы $F_{v}^{0}$ принадлежат пространству INT $H^{0}$ и образуют в нем тотальное подмножество $M^{0}$. При этом

$$
\left\langle F_{v_{1}}^{0}, F_{v_{2}}^{0}\right\rangle=c \exp \left(-\frac{1}{2} \operatorname{Re} \int_{X} \log \left(p\left(v_{1}(x), v_{2}(x)\right) d m(x)\right), \quad c=\exp \left(\frac{\gamma}{2}\right) .\right.
$$

Зададим операторы $U^{0}(g), g \in \mathrm{U}(n, 1)^{X}$, на $\tilde{M}^{0}$ равенством:

$$
U^{0}(g) F_{v}^{0}=\exp \left(\frac{1}{2} \operatorname{Re} \int_{X} \varphi(g(x), v(x)) d m(x)\right) F_{g v}^{0},
$$

где $\varphi(g, v)$ задается равенством (7.34). 
Подобно операторам $U^{ \pm}$, на элементах подгруппы $P^{X}$ эти операторы совпадают с операторами исходного представления $U^{0}$ группы $P^{X}$.

Введем, далее, по аналогии со случаем $U^{ \pm}$, тотальное множество $\widetilde{M}^{0} \subset$ INT $H^{0}$ функционалов вида

$\Psi_{g}^{0}=(2 c)^{-1 / 2} \exp \left(\frac{1}{2} \operatorname{Re} \int_{X} c\left(v(x), v_{0}\right) d m(x)\right) F_{v}^{0}, \quad g \in \mathrm{U}(n, 1)^{X}, \quad$ где $v=g v_{0}$.

ПрЕДЛОЖЕНИЕ 7.7. На множестве $\widetilde{M}^{0}$ скалярное произведение и операторы представления задаются формулами:

$$
\left\langle\Psi_{g_{1}}^{0}, \Psi_{g_{2}}^{0}\right\rangle=\exp \left(\int_{X}\left\langle b^{0}\left(g_{1}(x)\right), b^{0}\left(g_{2}(x)\right)\right\rangle d m(x)\right),
$$

где $b^{0}(g)-1-к о ц и к л ~ G \rightarrow \mathscr{H}^{0}$, заданный равенством

$$
\begin{gathered}
b^{0}(g)=2^{-1 / 2}(b(g), \overline{b(g)}) ; \\
U^{0}\left(g_{1}\right) \Psi_{g}^{0}=\exp \left(-\int_{X} \widetilde{u}\left(g_{1}(x), g(x)\right) d m(x)\right) \Psi_{g_{1} g}^{0},
\end{gathered}
$$

әде

$$
\widetilde{u}\left(g_{1}, g\right)=\frac{1}{2}\left\|b\left(g_{1}\right)\right\|^{2}+\left\langle\widetilde{T}^{0}\left(g_{1}\right) b^{0}(g), b^{0}\left(g_{1}\right)\right\rangle .
$$

Доказательство то же, что и для случая $\mathrm{SL}(2, \mathbb{R})$.

Отсюда по аналогии с теоремами 6.2 и 6.3 получаем следующую теорему.

Теорема 7.5. Операторы $U^{0}(g)$ сохраняют скалярные произведения $\left\langle\Psi_{g_{1}}^{0}, \Psi_{g_{2}}^{0}\right\rangle$ и продолжсатся до ортогональных (непроективных) операторов на всем пространстве INT $H^{0}$.

7.8. Связь между интегральной и фоковской моделями представления группы $\mathrm{U}(n, 1)^{X}$. Эта связь аналогична установленной ранее связи для случая группы $\mathrm{SL}(2, \mathbb{R})$.

Обозначим через $V^{ \pm}$фоковские проективные унитарные представления группы $\mathrm{U}(n, 1)^{X}$, отвечающие парам $\left(\widetilde{T}^{ \pm}, b^{ \pm}\right)$, где $\widetilde{T}^{ \pm}$- особые унитарные представления группы $\mathrm{U}(n, 1)$ и $b^{ \pm}: \mathrm{U}(n, 1) \rightarrow \mathscr{H}^{ \pm}-1$-коциклы, определенные равенством (7.16). Аналогично, обозначим через $V^{0}$ фоковское ортогональное представление, отвечающее паре $\left(\widetilde{T}^{0}, b^{0}\right)$.

Теорема 7.6. Продолжения на $\mathrm{U}(n, 1)^{X}$ интегральных моделей $\mathrm{INT}^{ \pm}$ унитарных представлений группы $P^{X}$ проективно эквивалентны фоковским проективным унитарным представлениям $V^{ \pm}$группы $\mathrm{U}(n, 1)^{X}$.

Продолжение на $\mathrm{U}(n, 1)^{X}$ интегралъной модели $\mathrm{INT}^{0}$ ортогонального представления группы $P^{X}$ эквивалентно фоковскому ортогональному представлению $V^{0}$ группь $\mathrm{U}(n, 1)^{X}$. Сплетающий оператор этих представлений порожден отображением ииклических векторов $\Psi_{e}^{0} \mapsto \operatorname{EXP} 0$.

ДокАзАтельство. По определению, фоковское представление $V^{+}$группы $\mathrm{U}(n, 1)$ реализуется в комплексном гильбертовом пространстве $\mathrm{EXP} \mathscr{H}^{X}$, где

$$
\operatorname{EXP} \mathscr{H}^{X}=\bigoplus_{k=0}^{\infty} S^{k} \mathscr{H}^{X}
$$


И

$$
\mathscr{H}^{X}=\int_{X}^{\oplus} \mathscr{H}_{x}^{+} d m(x), \quad \mathscr{H}_{x}^{+}=\mathscr{H}^{+},
$$

$\mathscr{H}^{+}-$пространство представления $\widetilde{T}^{+}$группы $P$.

Введем в ЕХР $\mathscr{H}^{X}$ тотальное подмножество $\mathscr{M}^{+} \subset \mathrm{EXP} \mathscr{H}^{X}$ векторов вида

$$
\Phi_{g}^{+}=\operatorname{EXP}^{X}(g), \quad g \in \mathrm{U}(n, 1)^{X},
$$

где $b^{X}: \mathrm{U}(n, 1)^{X} \rightarrow \mathscr{H}^{X}-1$-коцикл, порожденный 1 -коциклом $b^{+}: \mathrm{U}(n, 1) \rightarrow \mathscr{H}$. На нем скалярное произведение и операторы представления группы $\mathrm{U}(n, 1)^{X}$ задаются следующими формулами:

$$
\begin{aligned}
& \left\langle\Phi_{g_{1}}^{+}, \Phi_{g_{2}}^{+}\right\rangle=\exp \left(\int_{X}\left\langle b^{+}\left(g_{1}(x)\right), b^{+}\left(g_{2}(x)\right)\right\rangle d m(x)\right), \\
& V^{+}\left(g_{1}\right) \Phi_{g}^{+}=\exp \left(-\int_{X} u^{\prime}\left(g_{1}(x), g(x)\right) d m(x)\right) \Psi_{g_{1} g}^{+},
\end{aligned}
$$

где

$$
u^{\prime}\left(g_{1}, g\right)=\frac{i}{2} \operatorname{Im} c\left(g_{1} v_{0}, v_{0}\right)+\frac{1}{2}\left\|b\left(g_{1}\right)\right\|^{2}+\left\langle\widetilde{T}\left(g_{1}\right) b(g), b\left(g_{1}\right)\right\rangle
$$

Рассмотрим естественную биекцию $\widetilde{M}^{+} \rightarrow \mathscr{M}^{+}$тотальных подмножеств в пространствах INT $H^{+}$и $\mathrm{EXP} \mathscr{H}^{X}$. Из явных формул для скалярных произведений и операторов представлений $U^{+}(g)$ и $V^{+}(g)$ на $\widetilde{M}^{+}$и $\mathscr{M}^{+}$следует, что при этой биекции скалярные произведения сохраняются, а соответствующие операторы различаются только множителем:

$$
U^{+}(g)=\exp \left(-\frac{i}{2} \operatorname{Im} \int_{X} c\left(g(x) v_{0}, v_{0}\right) d m(x)\right) V^{+}(g) .
$$

Следовательно, представления $U^{+}$и $V^{+}$проективно эквивалентны. То же справедливо и для представлений $U^{-}$и $V^{-}$.

Аналогично, сравнивая формулы для скалярных произведений и операторов на тотальных подмножествах $\widetilde{M}^{0} \subset \mathrm{INT} H^{0}$ и $\mathscr{M}^{0} \subset \operatorname{EXP} \mathscr{H}^{X}$, убеждаемся, что эти формулы сохраняются при естественной биекции $\widetilde{M}^{0} \rightarrow \mathscr{M}^{0}$. Отсюда следует утверждение теоремы для случая представлений $U^{0}$ и $V^{0}$.

7.9. Добавление: унитарное представление группы $\widetilde{G}^{X}, G=\mathrm{U}(n, 1)$, где $\widetilde{G}$ - универсальная накрывающая группы $G=\mathrm{U}(n, 1)$. По определению, $\widetilde{G}$ является накрытием над $G$, где слой над $g \in G$ есть множество классов гомотопически эквивалентных путей на $G$ из единичного элемента $e$ в $g$. Элементы из $\widetilde{G}$ условимся обозначать через $\tilde{g}$, а их образы в $G$ - через $g$.

$\operatorname{KaK}$ и в случае $\mathrm{SL}(2, \mathbb{R})$, интегральные модели представлений $U^{ \pm}$группы токов $G^{X}=\mathrm{U}(n, 1)^{X}$ индуцирует представления $\widetilde{U}^{ \pm}$группы токов $\widetilde{G}^{X}$ в тех же гильбертовых пространствах $\widetilde{H}^{ \pm}$. Эти представления группы $\widetilde{G}^{X}$ проективно эквивалентны ее не проективным унитарным представлениям $V^{ \pm}$в тех же пространствах $\widetilde{H}^{ \pm}$, которые здесь будут явно описаны. Для определенности ограничимся описанием представления $V^{+}$. 
Конструкция представления $V^{+}$аналогична случаю $\mathrm{SL}(2, \mathbb{R})$. Достаточно определить операторы представления на элементах тотального подмножества функционалов $F_{v}^{+}$. Для этого сначала положим для любых $v=(a, b) \in L$ и $g=\left\|g_{i j}\right\|_{i, j=1,2,3} \in \mathrm{U}(n, 1)$ :

$$
\varphi(g, v)=-\log \left(g_{11}+g_{12} b+g_{13} a\right),
$$

где, как обычно, через log обозначена ветвь логарифма на плоскости с разрезом вдоль вещественной отрицательной полуоси такая, что $\log 1=0$. Эта функция $\varphi$ всюду конечна, и при любом фиксированном $v \in L$ она является однозначной аналитической функцией от $g \in G$ в достаточно малой окрестности единичного элемента. Поэтому для любого $g \in G$ и любого пути $\tilde{g}$ на $G$ из $e$ в $g$ определено ее аналитическое продолжение по этому пути из $e$ в $g$. Обозначим это аналитическое продолжение через $\psi(\tilde{g}, v)$. Определенная так функция $\psi(\tilde{g}, v)$ зависит только от гомотопического класса пути $\tilde{g}$, а потому является функцией на $\widetilde{G} \times L$.

Из определения следует:

$$
\psi(\tilde{g}, v)=-\log \left(g_{11}+g_{12} b+g_{13} a\right)
$$

при условии, что $g \in G$ и путь $\tilde{g}$ из $e$ в $g$ принадлежат достаточно малой окрестности единичного элемента $e$.

Подобно случаю $\mathrm{SL}(2, \mathbb{R})$, справедливо следующее утверждение.

ПреДЛОЖЕНИЕ 7.8. При любъх $\tilde{g}_{1}, \tilde{g}_{2} \in \widetilde{G} u v \in L^{+}$имеет место равенство

$$
\psi\left(\tilde{g}_{1} \tilde{g}_{2}, v\right)=\psi\left(\tilde{g}_{1}, g_{2} v\right)+\psi\left(\tilde{g}_{2}, v\right) .
$$

Поставим теперь в соответствие каждой паре $\tilde{g} \in \widetilde{G}^{X}$ и $v \in L^{X}$ следующую функцию на $X$ :

$$
\Psi_{\tilde{g}, v}(x)=\psi(\tilde{g}(x), v(x)) .
$$

Из предложения 7.8 следует, что функции $\Psi_{\tilde{g}, v}$ связаны соотношением:

$$
\Psi_{\tilde{g}_{1} \tilde{g}_{2}, v}=\Psi_{\tilde{g}_{1}, g_{2} v}+\Psi_{\tilde{g}_{2}, v}
$$

ОпрЕдЕлЕниЕ 17 . Определим действие операторов $V(\tilde{g}), \tilde{g} \in \widetilde{G}^{X}$, на функции $F_{v}$ тотального множества $M$ следующей формулой:

$$
V(\tilde{g}) F_{z}=\exp \left(\int_{X} \Psi_{\tilde{g}, v}(x) d m(x)\right) F_{g v} .
$$

Тогда, как и в случае $L(2, \mathbb{R})$, имеем следующую теорему.

Теорема 7.7. Операторы $V(\tilde{g})$ унитарны на $M$, m.e.

$$
\left\langle V(\tilde{g}) F_{v_{1}}, V(\tilde{g}) F_{v_{2}}\right\rangle=\left\langle F_{v_{1}}, F_{v_{2}}\right\rangle \quad \text { для любъх } v_{1}, v_{2} \in L^{X} u \tilde{g} \in \widetilde{G}^{X},
$$

и удовлетворяют соотношению

$$
V\left(\tilde{g}_{1} \tilde{g}_{2}\right) F_{v}=V\left(\tilde{g}_{1}\right) V\left(\tilde{g}_{2}\right) F_{v} \quad \text { для любых } \tilde{g}_{1}, \tilde{g}_{2} \in \widetilde{G}^{X} u v \in L^{X} .
$$

Тем самым, они порождают линейное унитарное представление группь $\widetilde{G}$ в пространстве $\widetilde{H}$. 
Очевидно, что построенное представление $V$ группы $\widetilde{G}^{X}$ проективно эквивалентно представлению $\widetilde{U}$ этой группы.

ЗАмечАниЕ. Другая модель унитарного представления группы $\widetilde{G}^{X}$ была построена в [32].

\section{8. Интегральные модели представлений группы $P^{X}$, где $P$ - максимальная параболическая подгруппа группы $\operatorname{Sp}(n, 1)$}

Здесь для случая группы $\operatorname{Sp}(n, 1)$ приведено описание канонических представлений подгруппы $P_{0} \subset P$; с каждым из них, в силу общей конструкции, ассоциировано неприводимое унитарное представление группы $P^{X}$. Отметим, что, в отличие от $\mathrm{O}(n, 1)$ и $\mathrm{U}(n, 1)$, эти представления не продолжаемы до представлений группы $\operatorname{Sp}(n, 1)^{X}$.

8.1. Исходные определения и обозначения. Реализуем $\operatorname{Sp}(n, 1)$ как группу линейных преобразований в $\mathbb{H}^{n+1}$, где $\mathbb{H}$ - тело кватернионов, сохраняющих форму $x_{1} \bar{y}_{n+1}+x_{n+1} \bar{y}_{1}+x_{2} \bar{y}_{2}+\cdots+x_{n} \bar{y}_{n}$ над $\mathbb{H}$, и будем представлять ее элементы в форме блочных матриц

$$
g=\left\|g_{i j}\right\|_{i, j=1,2,3},
$$

где на диагонали стоят квадратные матрицы соответственно порядка 1 , $n-1$ и 1.

В этой реализации

$$
P=D \wedge N
$$

где $N \cong \mathbb{R}^{n-1}-$ подгруппа клеточных матриц вида

$$
h=\left(\begin{array}{ccc}
1 & 0 & 0 \\
-w^{*} & e_{n-1} & 0 \\
t-\frac{w w^{*}}{2} & w & 1
\end{array}\right), \quad t \in \mathbb{H}_{0}, \quad w \in \mathbb{H}^{n-1}
$$

$\left(\mathbb{H}_{0}\right.$ - пространство мнимых кватернионов), а $D \cong \mathbb{H}^{*} \times \operatorname{Sp}(n-1)-$ подгруппа клеточно-диагональных матриц вида $d=\operatorname{diag}\left(\bar{s}^{-1}, u, s\right), s \in \mathbb{H}^{*}, u \in \operatorname{Sp}(n-1)$.

Представим $D$ в виде прямого произведения $D=\mathbb{R}_{+}^{*} \times D_{0}$, где $D_{0}$ - подгруппа матриц $d=\operatorname{diag}(\varepsilon, u, \varepsilon),|\varepsilon|=1$, и положим

$$
P_{0}=D_{0} \curlywedge N
$$

Таким образом,

$$
P=\mathbb{R}_{+}^{*} \lambda P_{0}=\left(\mathbb{R}_{+}^{*} \times D_{0}\right) \curlywedge N .
$$

Через $\operatorname{Sc} x$ и Vec $x$ будем обозначать соответственно вещественную и мнимую часть кватерниона $x$, т. е. Sc $x=(x+\bar{x}) / 2, \operatorname{Vec} x=(x-\bar{x}) / 2$.

Отождествим элементы из $D_{0}$ и $N$ соответственно с парами $(\varepsilon, u)$, где $\varepsilon \in$ $\operatorname{Sp}(1), u \in \operatorname{Sp}(n-1)$, и парами $(t, w)$, где $t \in \mathbb{H}_{0}, w \in \mathbb{H}^{n-1}$ (вектор-строка). Иногда будем также вместо $(t, w) \in N$ писать $(\zeta, w)$, где $\zeta=t-w w^{*} / 2$. 
В этих обозначениях групповые соотношения имеют вид:

$$
\begin{aligned}
\left(\zeta_{1}, w_{1}\right)\left(\zeta_{2}, w_{2}\right) & =\left(\zeta_{1}+\zeta_{2}-w_{1} w_{2}^{*}, w_{1},+w_{2}\right), \\
(\varepsilon, u)^{-1}(\zeta, w)(\varepsilon, u) & =(\bar{\varepsilon} \zeta \varepsilon, \bar{\varepsilon} z u), \\
r(\zeta, w) r^{-1} & =\left(r^{2} \zeta, r w\right) \quad \text { при } r \in \mathbb{R}_{+}^{*} .
\end{aligned}
$$

8.2. Описание канонических представлений группы $P_{0}$. Для описания канонических представлений группы $P_{0}$ сначала введем, подобно случаю группы $\mathrm{U}(n, 1)$, приводимое унитарное представление этой группы и покажем затем, что неприводимые составляющие этого представления являются каноническими.

Обозначим через $S^{2}$ пространство мнимых кватернионов $s$ с нормой 1 , изоморфное двумерной сфере $\left(s \in \mathbb{H}_{0}, s^{2}=-1\right)$. На $S^{2}$ естественно действует группа кватернионов $\varepsilon$ с нормой 1: $s \mapsto \bar{\varepsilon} s \varepsilon$.

Введем унитарное представление $T$ группы $P_{0}$ в гильбертовом пространстве функций $f(s, w)$ на $S^{2} \times \mathbb{H}^{n-1}$ с нормой

$$
\|f\|^{2}=\int_{S^{2}} \int_{\mathbb{H}^{n-1}}|f(s, w)|^{2} d \mu(w) d \mu(s),
$$

где $d \mu(w)$ - лебегова мера на $\mathbb{H}^{n-1} \cong \mathbb{R}^{4(n-1)}$ и $d \mu(s)$ - инвариантная мера на $S^{2}$.

Операторы представления имеют вид:

$$
\begin{aligned}
& (\widetilde{T}(g) f)(s, w)=\exp \left(-i \operatorname{Sc}\left[s\left(\zeta_{0}-w w_{0}^{*}\right)\right]\right) f\left(s, w+w_{0}\right) \quad \text { при } g=\left(\zeta_{0}, w_{0}\right) \in N \\
& (\widetilde{T}(g) f)(s, w)=f(\bar{\varepsilon} s \varepsilon, \bar{\varepsilon} w u) \quad \text { при } g=(\varepsilon, u) \in D_{0} .
\end{aligned}
$$

Групповое свойство и унитарность операторов сразу же следуют из этих формул. Очевидно, что операторы представлений $\widetilde{T}_{r}(g)=\widetilde{T}\left(r g r^{-1}\right)$, сопряженных с $T$ относительно группы автоморфизмов $\mathbb{R}_{+}^{*}$, задаются формулами:

$$
\begin{aligned}
& \left(\widetilde{T}_{r}(g) f\right)(s, w)=\exp \left(-i \operatorname{Sc}\left[s\left(r^{2} \zeta_{0}-r w w_{0}^{*}\right)\right]\right) f\left(s, w+r w_{0}\right) \text { при } g=\left(\zeta_{0}, w_{0}\right) \in N ; \\
& \left(\widetilde{T}_{r}(g) f\right)(s, w)=f(\bar{\varepsilon} s \varepsilon, \bar{\varepsilon} w u) \quad \text { при } g=(\varepsilon, u) \in D_{0} .
\end{aligned}
$$

Ассоциированное с $\widetilde{T}$ представление группы $P$ реализуется в прямом интеграле по мере $d^{*} r=r^{-1} d r$ на $\mathbb{R}_{+}^{*}$ гильбертовых пространств $H_{r}=H$ с заданными на них представлениями $\widetilde{T}_{r}$ подгруппы $P_{0}$,

$$
\mathscr{H}=\int_{0}^{\infty} H_{r} d^{*} r
$$

т. е. в расслоении над $\mathbb{R}_{+}^{*}$ со слоем $H_{r}$. Операторы подгруппы $P_{0}$ действуют на этом расслоении послойно, а операторы $\widetilde{T}\left(r_{0}\right), r_{0} \in \mathbb{R}_{+}^{*}$, - по формуле $\left(\widetilde{T}\left(r_{0}\right) f\right)(r)=f\left(r_{0} r\right)$.

ТЕОРема 8.1. Пространство Н является прямой суммой инвариантных попарно не эквивалентных неприводимых подпространств $H_{m}$ :

$$
H=\bigoplus_{m=0}^{\infty} H_{m}
$$


Для каждого $m \geqslant 0$ представление группы $P_{0}$ в пространстве $H_{m}$ канонично и обладает единственным, с точностью до множителя, почти инвариантным вектором:

$$
f_{m}(\omega, w)=l_{m}^{2 n-3}\left(w w^{*}\right) e^{-\frac{1}{2} w w^{*}},
$$

где $l_{m}^{2 n-3}(x)-$ полином Лагерра.

Для доказательства теоремы представим $H$ как прямой интеграл,

$$
H=\int_{S^{2}} H(\omega) d \omega
$$

гильбертовых пространств функций $f(w)$ на $\mathbb{H}^{n-1}$ с нормой

$$
\|f\|^{2}=\int_{\mathbb{H}^{n-1}}|f(w)|^{2} d \mu(w) .
$$

Очевидно, что эти пространства $H(\omega)$ инвариантны относительно подгруппы $P_{1}=D_{1} \prec N \subset P_{0}$, где $D_{1} \subset D_{0}$ - подгруппа элементов $(1, u)$, и что представления подгруппы $P_{1}$ преобразуются одно в другое действием подгруппы автоморфизмов $g \mapsto\left(\varepsilon, e_{n-1}\right)^{-1} g\left(\varepsilon, e_{n-1}\right),\left(\varepsilon, e_{n-1}\right) \in D_{0}$.

Утверждение теоремы 8.1 непосредственно следует из аналогичного утверждения для пространств $H(\omega)$ :

ПреДЛОЖЕНИЕ 8.1. Каждое пространство $Н(\omega)$ является прямой суммой инвариантных попарно не эквивалентных неприводимых относительно подгруппы $P_{1}$ подпространств $H_{m}(\omega)$ :

$$
H(\omega)=\bigoplus_{m=0}^{\infty} H_{m}(\omega) .
$$

Для каждого $m \geqslant 0$ представление группь $P_{1}$ в пространстве $H_{m}(\omega)$ канонично и обладает единственным, с точностью до множителя, почти инвариантным вектором:

$$
f_{m}(w)=l_{m}^{2 n-3}\left(w w^{*}\right) e^{-\frac{1}{2} w w^{*}}
$$

8.3. Доказательство предложения 8.1. Достаточно доказать утверждение для одного фиксированного $\omega \in S^{2}$, например, для $\omega=e_{1}$, где $e_{1}$ - базисный вектор в $\mathbb{H}$. Положим для краткости $H\left(e_{1}\right)=\mathscr{H}$.

Представим кватернионы $\sum_{k=0}^{3} a_{k} e_{k} \in \mathbb{H}$ как элементы алгебры над $\mathbb{C}: a=$ $\left(a_{0}+i a_{1}\right)+\left(a_{2}+i a_{3}\right) j$, где $j^{2}=-1$ и $i j=-j i$, и будем интерпретировать функции $f(w)$ на $\mathbb{H}^{n-1}$ как функции $f(z)=f\left(z^{1}, z^{2}\right)$ на $\mathbb{C}^{2 n-2}=\mathbb{C}^{n-1} \times \mathbb{C}^{n-1}$, где $w \in \mathbb{H}^{n-1}$ и $z=\left(z^{1}, z^{2}\right)$ связаны соотношением $w=z^{1}+z^{2} j$. Таким образом, в новой реализации представление группы $P_{1}$ действует в пространстве функций $f(z)$ на $\mathbb{C}^{2 n-2}$ с нормой

$$
\|f\|^{2}=\int_{\mathbb{C}^{2 n-2}}|f(z)|^{2} d \mu(z) .
$$


Лемма. В новой реализачии операторы представления группъ $P_{1}$ имеют следующий вид:

$$
(T(g) f)\left(t_{0}, w_{0}\right)=\exp \left(i\left(t_{1}-\operatorname{Im}\left(z z_{0}^{*}\right)\right)\right) f\left(z+z_{0}\right) \quad n p u g=\left(t_{0}, w_{0}\right) \in N,
$$

где $t_{1} \in \mathbb{R} u z_{0}=\left(z_{0}^{1}, z_{0}^{2}\right) \in \mathbb{C}^{2 n-2}$ определяются из соотношений $t_{0}=t_{1} e_{1}+$ $t_{2} e_{2}+t_{3} e_{3} u w_{0}=z_{0}^{1}+z_{0}^{2} j$

$$
(T(g) f)(z)=f(z v) \quad \text { npu } g=(1, u) \in D_{1},
$$

¿de

$$
v=\left(\begin{array}{cc}
u_{1} & u_{2} \\
-u_{2}^{\prime} & u_{1}^{\prime}
\end{array}\right) \quad \text { прu } u=u_{1}+u_{2} j \in \operatorname{Sp}(n-1)
$$

(итрих обозначает транспонирование).

ДокАЗАТЕЛЬство. При $g=\left(t_{0}, w_{0}\right) \in N$ оператор $T(g)$ в исходной реализации имеет вид:

$$
(\widetilde{T}(g) f)(w)=\exp \left(-i \operatorname{Sc}\left[e_{1}\left(t_{0}-w w_{0}^{*}\right)\right]\right) f\left(w+w_{0}\right) .
$$

Очевидно, что в новой реализации $\operatorname{Sc}\left(e_{1} t_{0}\right)=-t_{1}$. Имеем, далее: $w w_{0}^{*}=$ $z^{1}\left(z_{0}^{1}\right)^{*}+z^{2}\left(z_{0}^{2}\right)^{*}+z^{1}\left(z_{0}^{2} j\right)^{*}+\left(z_{0}^{2} j\right)\left(z^{1}\right)^{*}$. Легко убедиться, что $\operatorname{Sc}\left[e_{1}\left(z^{1}\left(z_{0}^{2} j\right)^{*}+\right.\right.$ $\left.\left.\left(z_{0}^{2} j\right)\left(z^{1}\right)^{*}\right)\right]=0$. Поэтому $\operatorname{Sc}\left[e_{1}\left(w w_{0}^{*}\right)\right]=\operatorname{Re}\left(i z^{1}\left(z_{0}^{1}\right)^{*}+z^{2}\left(z_{0}^{2}\right)^{*}\right)=-\operatorname{Im}\left(z z_{0}^{*}\right)$. Отсюда следует (8.5).

Далее, при $g=(1, u) \in D_{1}$ оператор $T(g)$ в исходной реализации имеет вид:

$$
(\widetilde{T}(g) f)(w)=f(w u)
$$

Имеем: $w u=\left(z^{1}+z^{2} j\right)\left(u_{1}+u_{2} j\right)=z^{1} u_{1}+z^{2}\left(j u_{2} j\right)+\left(z^{1} u_{2}-z^{2}\left(j u_{1} j\right)\right) j$. Таким образом, поскольку $j u_{i} j=-u_{i}^{\prime}$, вектору $w u \in \mathbb{H}^{n-1}$ соответствует вектор $\left(z^{1}, z^{2}\right) v$, где $v$ - клеточная матрица вида (8.7). Лемма доказана.

Обозначим через $V_{n}$ группу всех преобразований $v$ вида (8.7) в пространстве $\mathbb{C}^{2 n-2}$. Очевидно, что $V_{n} \equiv \mathrm{Sp}(n-1)$.

Убедимся, что $V_{n} \subset U(n-1)$.

В самом деле, условие $u \in \operatorname{Sp}(n-1)$ эквивалентно условию

$$
\left(u_{1}+u_{2} j\right)\left(u_{1}+u_{2} j\right)^{*}=e_{m-1},
$$

что, в свою очередь, эквивалентно соотношениям $u_{1} u_{1}^{*}+u_{2} u_{2}^{*}=e_{n-1}$ и $u_{1} \bar{u}_{2}=$ $u_{2} \bar{u}_{1}$, где черта обозначает комплексное сопряжение. Из этих соотношений непосредственно следует принадлежность матриц вида (8.7) группе $U(2 n-2)$.

Полученные формулы для операторов $T(g)$ представления группы $P_{1} \subset$ $\operatorname{Sp}(n, 1)$ совпадают с формулами (8.4) и (8.5) для операторов $T^{+}$представления подгруппы $P_{0}$ для случая $\mathrm{U}(n, 1)$, в которых лишь $n$ должно быть заменено на $2 n-1$. Поэтому разложение представления на неприводимые канонические представления может быть проведено далее по той же схеме.

Сначала перейдем к новой реализации этого представления, положив

$$
f(z)=\varphi(z) \exp \left(-\frac{z z^{*}}{2}\right) .
$$


В новой реализации представление действует в гильбертовом пространстве функций $f(z)$ с нормой

$$
\|f\|^{2}=\int_{\mathbb{C}^{2 n-2}}|f(z)|^{2} \exp \left(-z z^{*}\right) d \mu(z) .
$$

При этом формулы (8.6) для операторов $T(g), g \in D_{1}$, сохраняются, а формулы для операторов $T(g), g=\left(t_{0}, z_{0}\right) \in N$, принимают вид:

$$
(T(g) f)(z)=\exp \left(i \frac{t_{1}-z_{0} z_{0}^{*}}{2}-z z_{0}^{*}\right) f\left(z+z_{0}\right) .
$$

В этой реализации мультипликатор в формуле для оператора $T(g), g \in N$, является целой аналитической функцией от $z$. Отсюда, как и в случае $\mathrm{U}(n, 1)$, следует, что пространство представления $\mathscr{H}$ разлагается в прямую сумму неприводимых попарно не эквивалентных инвариантных подпространств:

$$
\mathscr{H}=\bigoplus_{m=0}^{\infty} H_{m}
$$

где $H_{m}$ - подпространство, циклически порожденное однородными полиномами степени $m$ от $\bar{z}_{1}, \bar{z}_{2}, \ldots, \bar{z}_{2 n-2}$.

Отметим, что в случае $\mathrm{U}(n, 1)$ неприводимость подпространств $H_{m}$ следовала из неприводимости пространства однородных полиномов от $\bar{z}_{1}, \bar{z}_{2}, \ldots, \bar{z}_{2 n-2}$ относительно действия всей унитарной группы $U(2 n-2)$. Однако это свойство сохраняется и при замене группы $U(2 n-2)$ ее подгруппой $V_{n} \equiv \operatorname{Sp}(n-1)$.

Далее, так же, как и в случае $\mathrm{U}(n, 1)$, доказывается, что в каждом пространстве $H_{m}$ существует и притом единственный почти инвариантный вектор, равный $l_{m}^{2 n-3}\left(z z^{*}\right)$.

Поскольку $z z^{*}=w w^{*}$, то в исходной реализации представления и в исходных координатах $w$ этот вектор задается формулой (8.4).

\section{Список литературы}

[1] А. М. Вершик, И. М. Гельфанд, М. И. Граев, "Представления группы $S L(2, \mathbf{R})$, где $\mathbf{R}$ - кольцо функций", УМH, 28:5 (1973), 83-128; англ. пер.: А. M. Vershik, I. M. Gel'fand, M. I. Graev, "Representations of the group $S L(2, \mathbf{R})$, where $\mathbf{R}$ is a ring of functions", Russian Math. Surveys, 28:5 (1973), 87-132.

[2] А. М. Вершик, И. М. Гельфанд, М. И. Граев, "Неприводимые представления группы $G^{X}$ и когомологии", Функи. анализ и его прил., 8:2 (1974), 67-69; англ. пер.: A. M. Vershik, I. M. Gel'fand, M. I. Graev, "Irreducible representations of the group $G^{X}$ and cohomologies", Funct. Anal. Appl., 8:2 (1974), 151-153.

[3] H. Araki, "Factorizable representation of current algebra. Non commutative extension of the Lévy-Khinchin formula and cohomology of a solvable group with values in a Hilbert space", Publ. Res. Inst. Math. Sci., 5:3 (1970), 361-422.

[4] A. Guichardet, Cohomologie des groupes topologiques et des algèbres de Lie, Textes Math., 2, CEDIC, Paris, 1980; пер. с франц.: А. Гишарде, Когомологии топологических групп и алгебр Ли, Мир, М., 1984.

[5] Д.А. Каждан, "О связи дуального пространства группы со строением ее замкнутых подгрупп”, Функи. анализ и его прил., 1:1 (1967), 71-74; англ. пер.: D. A. Kazhdan, "Connection of the dual space of a group with the structure of its close subgroups", Funct. Anal. Appl., 1:1 (1967), 63-65. 
[6] B. Bekka, P. de la Harpe, A. Valette, Kazhdan's property (T), New Math. Monogr., 11, Cambridge Univ. Press, Cambridge, 2008.

[7] А.М. Вершик, С.И. Карпушев, "Когомологии групп в унитарных представлениях, окрестность единицы и условно положительно определенные функции", Матем. сб., 119:4 (1982), 521-533; англ. пер.: А. M. Vershik, S. I. Karpushev, "Cohomology of groups in unitary representations, the neighborhood of the identity, and conditionally positive definite functions", Math. USSR-Sb., 47:2 (1984), 513-526.

[8] А. М. Вершик, И. М. Гельфанд, М.И. Граев, "Представления группы диффеоморфизмов", УМH, 30:6 (1975), 3-50; англ. пер.: А. M. Vershik, I. M. Gel'fand, M. I. Graev, "Representations of the group of diffeomorphisms and conditionally positive definite functions", Russian Math. Surveys, 30:6 (1975), 1-50.

[9] A. M. Vershik, I. M. Gel'fand, M.I. Graev, "Representations of the group of smooth mappings of a manifold $X$ into a compact Lie group", Compositio Math., 35:3 (1977), 299-334.

[10] A. M. Veršik, I. M. Gel'fand, M. I. Graev, "Representations of the group of functions taking values in a compact Lie group", Compositio Math., 42:2 (1980), 217-243.

[11] А. М. Вершик, И. М. Гельфанд, М.И. Граев, "Коммутативная модель представления группы токов $S L(2, \mathbb{R})^{X}$, связанная с унипотентной подгруппой”, Функи. анализ и его прил., 17:2 (1983), 70-72; англ. пер.: A. M. Vershik, I. M. Gel'fand, M. I. Graev, "A commutative model of representation of the group of flows $S L(2, \mathbb{R})^{X}$ that is connected with a unipotent subgroup", Funct. Anal. Appl., 17:2 (1983), 137-139.

[12] I. M. Gelfand, M.I. Graev, A. M. Vershik, "Models of representations of current groups", Representations of Lie groups and Lie algebras (Budapest, 1971), Akad. Kiadó, Budapest, 1985, 121-179.

[13] А. М. Вершик, М.И. Граев, "Коммутативная модель представления группы $O(n, 1)^{X}$ и обобщенная лебегова мера в пространстве распределений”, Функи. анализ и его прил., 39:2 (2005), 1-12; англ. пер.: M. I. Graev, A. M. Vershik, "A commutative model of a representation of the group $O(n, 1)^{X}$ and a generalized Lebesgue measure in the space of distributions", Funct. Anal. Appl., 39:2 (2005), 81-90.

[14] M. I. Graev, A. M. Vershik, "The basic representation of the current group $O(n, 1)^{X}$ in the $L^{2}$ space over the generalized Lebesgue measure", Indag. Math. (N. S.), 16:3-4 (2005), 499-529.

[15] А.М. Вершик, М.И. Граев, "Интегральные модели представлений групп токов", Функи. анализ и его прил., 42:1 (2008), 22-32; англ. пер.: А. M. Vershik, M. I. Graev, "Integral models of representations of current groups", Funct. Anal. Appl., 42:1 (2008), 19-27.

[16] А. М. Вершик, М.И. Граев, "Интегральные модели унитарных представлений групп токов со значениями в полупрямых произведениях", Функи. анализ и его прил., 42:4 (2008), 37-49; англ. пер.: А. M. Vershik, M. I. Graev, "Integral models of unitary representations of current groups with values in semidirect products", Funct. Anal. Appl., 42:4 (2008), 279-289.

[17] P. Delorme, "Irreductibilité de certaines représentations de $G^{X}$ ", J. Funct. Anal., 30:1 (1978), 36-47.

[18] R.S. Ismagilov, "Representations of infinite-dimensional groups", Transl. Math. Monogr., 152, Amer. Math. Soc., Providence, RI, 1996.

[19] А. М. Вершик, “Существует ли бесконечномерная мера Лебега?", Анализ и особенности. Часть 2, Тр. МИАН, 259, Наука, М., 2007, 256-281; англ. пер.: A.M. Vershik, "Does there exist a Lebesgue measure in the infinite-dimensional space?", Proc. Steklov Inst. Math., 259:2 (2007), 248-272.

[20] A. M. Vershik, "The behavior of the Laplace transform of the invariant measure on the hypersphere of high dimension", J. Fixed Point Theory Appl., 3:2 (2008), 317-329. 
[21] A. M. Vershik, "Invariant measures for the continual Cartan subgroup", J. Funct. Anal., 255:9 (2008), 2661-2682.

[22] N. Tsilevich, A. Vershik, M. Yor, "An infinite-dimensional analogue of the Lebesgue measure and distinguished properties of the gamma process", J. Funct. Anal., 185:1 (2001), 274-296.

[23] A. Vershik, B. Tsirelson, "Examples of nonlinear continuous tensor product of measure spaces and non-Fock factorisations", Rev. Math. Phys., 10:1 (1998), 81-145.

[24] А. М. Вершик, М.И. Граев, "Структура дополнительных серий и особых представлений групп $O(n, 1)$ и $U(n, 1)$ ", УMH, 61:5 (2006), 3-88; англ. пер.: А. M. Vershik, M. I. Graev, "Structure of the complementary series and special representations of the groups $O(n, 1)$ and $U(n, 1)$ ", Russian Math. Surveys, 61:5 (2006), 799-884.

[25] И. М. Гельфанд, М. И. Граев, "Особые представления группы SU(n, 1) и проективные унитарные представления группы токов $\mathrm{SU}(\mathrm{n}, 1)^{\mathrm{X}}$ ”, Докл. АН CCCP, 332:3 (1993), 280-282; англ. пер.: I. M. Gel'fand, M. I. Graev, "Special representations of the group $\mathrm{SU}(n, 1)$ and projective unitary representations of the current group $\mathrm{SU}(n, 1)^{X}$, Russian Acad. Sci. Dokl. Math., 48:2 (1994), 291-295.

[26] В.Ф. Молчанов, "Канонические представления и надгруппы для гиперболоидов", Функи. анализ и его прил., 39:4 (2005), 48-61; англ. пер.: V. F. Molchanov, "Canonical representations and overgroups for hyperboloids", Funct. Anal. Appl., 39:4 (2005), 284-295.

[27] А. М. Переломов, Обобщенные когерентные состояния и их применения, Наука, М., 1987; пер. с англ.: А. Perelomov, Generalized coherent states and their applications, Texts Monogr. Phys., Springer-Verlag, Berlin, 1986.

[28] A. Guichardet, "Symmetric Hilbert space and related topics", Lecture Notes in Math., 261, Springer-Verlag, Berlin-New York, 1972.

[29] P. Delorme, "1-cohomologie des représentations unitaires des groupes de Lie semisimples et résolubles. Produits tensoriels continus de représentations", Bull. Soc. Math. France, 105:3 (1977), 281-336.

[30] А.М. Вершик, Н.В. Цилевич, "Фоковские факторизации и разложения пространства $L^{2}$ над общим процессом Леви”, УМH, 58:3 (2003), 3-50; англ. пер.: A. M. Vershik, N.V. Tsilevich, "Fock factorizations, and decompositions of the $L^{2}$ spaces over general Lévy processes", Russian Math. Surveys, 58:3 (2003), 427-472.

[31] K. R. Parthasarathy, K. Schmidt, Positive definite kernels, continuous tensor products, and central limit theorems of probability theory, Lecture Notes in Math., 272, Springer-Verlag, Berlin-New York, 1972.

[32] Ф. А. Березин, "Представления непрерывного прямого произведения универсальных накрывающих группы движений комплексного шара”, Tp. MMO, 36 (1978), 275-293; англ. пер.: F. A. Berezin, "Representations of a continuous direct product of universal coverings of the group of motions of the complex ball", Trans. Moscow Math. Soc., 36 (1979), 281-298.

А. М. Вершик (А. M. Vershik)

Поступила в редакцию

Санкт-Петербургское отделение

24.12.2008

Математического института им. В. А. Стеклова РАН

E-mail: vershik@pdmi.ras.ru

М. И. Граев (М. I. Graev)

Научно-исследовательский институт

системных исследований РАН

E-mail: graev_36@mtu-net.ru 